Historic, archived document

Do not assume content reflects current scientific knowledge, policies, or practices. 

Information contained in this report includes the most commonly used Forest Inventory and Analysis (FIA) statistics. However, additional forest resource data can be provided to interested users. Persons requesting additional information that can be provided from the raw inventory data are expected to pay for the retrieval costs. These costs will vary, depending on the complexity of the request, from less than $\$ 100$ for a simple request to $\$ 2,000$ for a complete retrieval involving the services of a FIA computer programmer. If requests for data conflict with ongoing work, they will be scheduled so as to minimize the impact on the work unit.

Address requests for unpublished information to:

Burton L. Essex

Forest Inventory and Analysis Project

North Central Forest Experiment Station

1992 Folwell Avenue

St. Paul, Minnesota 55108

Phone: (612) 642-5275

Area served: Illinois, Indiana, Iowa, Kansas, Michigan, Minnesota, Missouri, Nebraska, North Dakota, eastern South Dakota, Wisconsin.

North Central Forest Experiment Station

Forest Service--U.S. Department of Agriculture

1992 Folwell Avenue

St. Paul, Minnesota 55108

Manuscript approved for publication June 17, 1985

1985 


\section{FOREWORD}

Forest Inventory and Analysis (FIA) is a continuing endeavor as mandated by the Forest and Rangeland Renewable Resources Planning Act of 1974. Prior inventories were mandated by the McSweeney-McNary Forest Research Act of 1928. The objective of FIA is to periodically inventory the Nation's forest land to determine its extent, condition, and volume of timber, growth, and depletions. Up-to-date resource information is essential to frame intelligent forest policies and programs. USDA Forest Service regional experiment stations are responsible for conducting these inventories and publishing summary reports for individual States. The North Central Forest Experiment Station is responsible for Forest Inventory and Analysis work in Illinois, Indiana, Iowa, Kansas, Michigan, Minnesota, Missouri, Nebraska, North Dakota, eastern South Dakota, and Wisconsin.

Fieldwork for the 1983 Nebraska Forest Survey began in April 1982 and was completed in March 1983. The previous survey of Nebraska timber resources was dated 1955. The Nebraska Forest Service canvassed mills and provided the fuelwood and post statistics.

Aerial photos used in this inventory were furnished by the USDA Agricultural Stabilization and Conservation Service offices in each county. 


\section{CONTENTS}

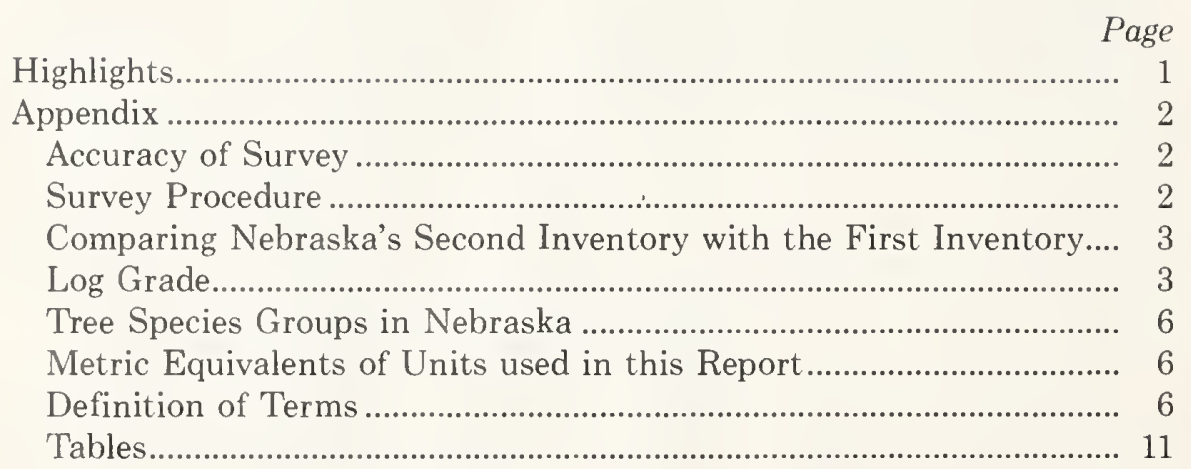




\title{
NEBRASKA FOREST STATISTICS, 1983
}

\author{
Gerhard K. Raile, Mensurationist
}

\section{HIGHLIGHTS}

\section{Area}

- Forest land area totaled 718,300 acres--1.5 percent of the State's total land area.

- Twenty-three percent of the State's forest land is unproductive.

- Commercial forest land decreased by 25 percent between 1955 and 1983, from 714,700 to 537,800 acres.

- Farmers own 71 percent of the State's commercial forest land.

- Forty percent of the commercial forest land is in sawtimber stands of ponderosa pine $(129,700$ acres $)$ and elm-ash-cottonwood (85,800 acres).

- Sawtimber stands account for 69 percent of the commercial forest followed by poletimber stands ( 17 percent), sapling and seedling stands (13 percent), and nonstocked areas (1 percent).

- Stands between 60 and 101 years of age occupy 48 percent of the commercial forest.

- Thirty-nine percent of the commercial forest is poorly stocked (16.7 to 60 percent), 49 percent is medium stocked (61 to 100 percent), and 10 percent is well stocked (101 to 133 percent) with growingstock trees.

- Wooded strips occupy 262,200 acres. Wooded strips are not classified as commercial forest because they are too narrow to meet the definition.

- Windbreaks amount to 139,100 acres and are part of the $1,111,700$ acres of nonforest land with trees in the State.

\section{Volume}

- The total volume of timber on commercial forest land in 1983 was 561.6 million cubic feet--456.0 million in growing-stock trees, 96.3 million in cull trees, and 9.3 million in salvable dead trees.

- Growing-stock volume increased 23 percent from 370.2 million cubic feet in 1955 to 456.0 million cubic feet in 1983.

- Growing-stock volume per acre increased 64 percent from 518 cubic feet in 1955 to 848 cubic feet in 1983.
- Elm growing stock dropped drastically due to Dutch elm disease--from 51.3 million cubic feet in 1955 to 12.4 million cubic feet in 1983.

- Ponderosa pine is the species with the most sawtimber volume on commercial forest land (46 percent of the total), followed by cottonwood (30 percent) and bur oak ( 7 percent).

- Farmers own 78 percent of the growing-stock volume (327.4 million cubic feet).

- The ponderosa pine type accounts for 34 percent, the cottonwood type for 23 percent, and the elmash-cottonwood type for 19 percent of the growingstock volume.

- The ponderosa pine type has the most sawtimber volume per acre with 5,216 board feet.

\section{Stand Conditions}

- Net annual growth of growing stock amounted to 11.1 million cubic feet in 1982, 2.4 percent of inventory.

- Sawtimber growth was 42.7 million board feet in 1982, 2.5 percent of inventory.

- Mortality of growing-stock trees totaled 2.2 million cubic feet in 1982, 0.5 percent of inventory.

- Weather accounts for 17 percent of the sawtimber mortality.

- Almost two-thirds of the sawtimber volume by butt log grade is concentrated in log grade 3 .

\section{Timber Use}

- Timber removals from growing stock totaled 9.4 million cubic feet in 1982--8.4 million for roundwood products, 440,000 for logging residue, and 513,000 for other removals.

- Removals of growing stock for 1982 were 147 percent higher than those in 1953.

- Farmers and miscellaneous private individuals owned 99 percent of the 1982 growing-stock removals.

- Sawtimber removals from commercial forest land totaled 36.3 million board feet in 1982, 102 percent higher than in 1953.

- Growing-stock removals for roundwood products totaled 8,397,000 cubic feet-- 62 percent as firewood, 35 percent as saw logs, and 3 percent as other products. 
- Wood residue from primary plants amounted to $1,647,300$ cubic feet--69 percent of it was used for domestic fuel and products such as livestock bedding, and mulch.

\section{Biomass}

- Live tree biomass (trees greater than 1 inch d.b.h.) totaled 29 million green tons or 54 tons per acre of commercial forest land.
- Highest yields per acre of live tree biomass are in the cottonwood type (72 tons) and the oak-hickory type (67 tons).

- Sixty-six percent of the live tree biomass is in the boles of trees greater than 5 -inches d.b.h., 27 percent is in the tops and limbs of these trees, and 7 percent is in trees less than 5 inches d.b.h.

\section{ACCURACY OF SURVEY}

Forest Inventory and Analysis information is based on a sampling procedure designed to provide reliable statistics at the State level. Consequently, the reported figures are estimates only. The sampling errors mean the chances are two out of three that the true inventory value is within the limits indicated.

For example, the estimated growing-stock volume in Nebraska in 1983, 456.0 million cubic feet, has a sampling error of \pm 8.6 percent $( \pm 39.2$ million cubic feet). Therefore, the growing-stock volume from a 100 percent inventory would have a two in three chance of falling between 416.8 and 495.2 million cubic feet.

The following tabulation shows the sampling errors for the 1983 Nebraska inventory:

\section{Item}

Growing stock
Volume
Growth
Removals
Sawtimber
Volume
Growth
Removals
Commercial forest
area

\section{Unit totals}

(Thousand cubic feet) 456,011

11,099

9,350

(Thousand board feet)

$1,711,873$

42,729

36,264

(Thousand acres)

537.8

\section{Sampling error}

(Percent)

8.6

10.9

20.6

9.4

14.3

20.9

6.0
As survey data are broken down into smaller sections, the sampling error increases. The smaller the breakdown, the larger the sampling error. For example, the sampling error for growing-stock volume in a particular county is higher than that for total growingstock volume in the State (table 89 shows the sampling errors for smaller estimates).

\section{SURVEY PROCEDURE}

The major steps in the survey of Nebraska were as follows:

1. A total of 260,180 1-acre points were systematically distributed across aerial photos of the entire
State. To make a preliminary estimate of forest area, photo interpretors classified these points as forest land $(5,564)$, nonforest land with trees $(4,770)$, nonforest land without trees $(247,873)$, questionable (69), and water $(1,904)$. Next, all of the forest, nonforest with trees, and questionable points were stereoclassified as to forest type, stand-size class, and density. Then 306 points classed as forest, 3 points classes as questionable, and 273 points classed as nonforest with trees were examined on the ground to correct the preliminary area estimate for errors in classification and for actual changes in land use since the aerial photos were taken. At each of 159 commercial forest locations, variable-radius plots (basal area factor 37.5 ) were established at 10 points uniformly placed over the sample acre. Tree measurements made at commercial forest locations were the basis for estimates of timber volume, growth, mortality, number of trees, and other forest classifications.

2. Statistics on timber utilization during 1980 were obtained from mill surveys. The Nebraska Forest Service canvassed resident sawmills and other primary wood-using plants. The North Central Forest Experiment Station canvassed out-of-State primary woodusing mills such as sawmills and veneer mills to determine their use of timber from Nebraska. The $\mathrm{Ne}$ braska Forest Service supplied 1980 estimates of fuelwood and post production from roundwood.

3. Estimates of primary mill residue used for fuelwood were obtained from the canvass of Nebraska primary wood-using plants. Timber cut for products by ownership class was determined by a canvass of public and forest industry timber owners. The portion of timber cut unaccounted for by the latter owners was grouped under "farmer and other owners".

4. Wood utilization factors for converting fuelwood, posts, and timber products output to removals from growing-stock were obtained during the 1971-1972 Missouri utilization study.

5. Field data were sent to St. Paul, Minnesota, to be processed and analyzed. 


\section{COMPARING NEBRASKA'S SECOND} SURVEY WITH THE FIRST SURVEY

Data from new forest surveys are often compared with data from earlier ones to determine trends in forest areas and volumes. Changes in procedures and definitions between surveys make it necessary to adjust earlier survey data so they are comparable to data from the new survey.

The published 1955 area of commercial forest land, $1,050,800$ acres, was adjusted due to definitional changes between surveys. Some land now defined as nonforest with trees was defined as commercial forest land in 1955. Also, new site productivity curves for ponderosa pine caused some land previously classified as commercial to be classified as unproductive forestland in the current survey. The adjusted 1955 area (705,700 acres) can be compared directly with the 1983 area $(537,800$ acres $)$.

The Timber Resource Analysis System (a USDA Forest Service computer program for updating, backdating, and projecting timber volume, growth, mortality, and removals) recalculated 1955 volumes using 1983 estimates of cubic foot volume per tree and 1983 board foot-cubic foot ratios. This adjustment was necessary so that volume differences between surveys would represent actual changes and not merely changes in the volume equations used on each occasion.

\section{LOG GRADE}

In Nebraska the butt $\log$ of every sawtimber tree sampled was graded for quality. Logs were graded on the basis of external characteristics. Hardwood species were graded according to "Hardwood Log Grades for Standard lumber"'. The best 12 -foot section of the lowest 16 -foot hardwood log, or the best 12 -foot upper section if the butt log did not meet minimum log-grade standards, was graded as follows:

'Vaughn, C. L.; Wollin, C. A.; McDonald, K. A.; Bulgrin, E. H. Hardwood log grades for standard lumber. Res. Pap. FPL-63. Madison, WI: U.S. Department of Agriculture, Forest Service, Forest Products Laboratory; 1966.52 p. 
Forest Service standard grades for hardwood factory saw logs

\begin{tabular}{|c|c|c|c|c|c|c|c|c|c|}
\hline \multirow{2}{*}{\multicolumn{2}{|c|}{ Grading factors }} & \multicolumn{8}{|c|}{ Specifications } \\
\hline & & \multicolumn{3}{|c|}{ Log grade 1} & \multicolumn{4}{|c|}{ Log grade 2} & \multirow{2}{*}{\begin{tabular}{|c|} 
Log grade 3 \\
$\begin{array}{c}\text { Butts and } \\
\text { uppers }\end{array}$
\end{tabular}} \\
\hline \multicolumn{2}{|l|}{ Position in tree } & $\begin{array}{l}\text { Butts } \\
\text { only }\end{array}$ & \multicolumn{2}{|c|}{$\begin{array}{c}\text { Butts and } \\
\text { uppers }\end{array}$} & & \multicolumn{3}{|c|}{ Butts and uppers } & \\
\hline \multicolumn{2}{|c|}{ Scaling diameter, inches } & $113-15$ & $16-19$ & $20+$ & ${ }^{2} 11+$ & & $12+$ & & $8+$ \\
\hline \multicolumn{2}{|c|}{ Length without trim, feet } & & $10+$ & & $10+$ & $8-9$ & $10-11$ & $12+$ & $8+$ \\
\hline \multirow{3}{*}{$\begin{array}{l}\text { Required } \\
\text { clear cuttings }{ }^{3} \\
\text { of each of three } \\
\text { best faces }\end{array}$} & Min. length, feet & 7 & 5 & 3 & 3 & 3 & 3 & 3 & 2 \\
\hline & Max. number & 2 & 2 & 2 & 2 & 2 & 2 & 3 & No Limit \\
\hline & $\begin{array}{l}\text { Min. proportion } \\
\text { of log length } \\
\text { required in } \\
\text { clear cutting }\end{array}$ & $5 / 6$ & $5 / 6$ & $5 / 6$ & $2 / 3$ & $3 / 4$ & $2 / 3$ & $2 / 3$ & $1 / 2$ \\
\hline \multirow{2}{*}{$\begin{array}{l}\text { Maximum } \\
\text { sweep and crook } \\
\text { allowance }\end{array}$} & $\begin{array}{l}\text { For logs with less } \\
\text { than one-fourth } \\
\text { of end in sound } \\
\text { defects }\end{array}$ & \multicolumn{3}{|c|}{15 percent } & & \multicolumn{3}{|c|}{30 percent } & 50 percent \\
\hline & $\begin{array}{l}\text { For logs with more } \\
\text { than one-fourth } \\
\text { of end in sound } \\
\text { defects }\end{array}$ & \multicolumn{3}{|c|}{10 percent } & & \multicolumn{3}{|c|}{20 percent } & 35 percent \\
\hline \multicolumn{2}{|c|}{ Maximum scaling deduction } & \multicolumn{3}{|c|}{40 percent $^{5}$} & & \multicolumn{3}{|c|}{50 percent $^{6}$} & 50 percent \\
\hline
\end{tabular}

${ }^{1}$ Ash and basswood butts can be 12 inches if they otherwise meet requirements for small \#1's.

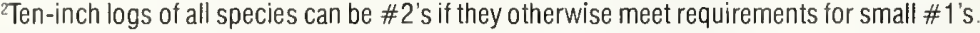

${ }^{3} \mathrm{~A}$ clear cutting is a portion of a face, extending the width of the face, that is free of defects.

${ }^{4} \mathrm{~A}$ face is one-four th of the surface of the log as divided lengthwise.

${ }^{5} 0$ therwise \#1 logs with 41-60 percent deductions can be \#2.

${ }^{6} 0$ therwise \#2 $\log$ s with 51-60 percent deductions can be \#3. 


\section{Forest Service standard specifications for hardwood construction logs (tie and timber logs)}

\begin{tabular}{|c|c|c|}
\hline \multicolumn{2}{|l|}{ Position in tree } & Butt and upper \\
\hline \multicolumn{2}{|l|}{ Min. diameter, small end } & 8 inches + \\
\hline \multicolumn{2}{|c|}{ Min. length, without trim } & 8 feet \\
\hline \multicolumn{2}{|l|}{ Clear cuttings } & No requirements. \\
\hline \multicolumn{2}{|c|}{ Sweep allowance, absolute } & $\begin{array}{l}\text { One-fourth of the diameter at the small end for each } 8 \\
\text { feet of length. }\end{array}$ \\
\hline & Single knots & $\begin{array}{l}\text { Any number, if no one knot has an average diameter } \\
\text { above the callus in excess of one-third of the log diame- } \\
\text { ter at point of occurrence. }\end{array}$ \\
\hline \multirow[t]{2}{*}{ Sound surface defects } & Whorled knots & $\begin{array}{l}\text { Any number if sum of knot diameters above the callus } \\
\text { does not exceed one-third of the log diameter at point of } \\
\text { occurrence. }\end{array}$ \\
\hline & Holes & $\begin{array}{l}\text { Any number provided none has a diameter over one- } \\
\text { third of the log diameter at point of occurrence, and } \\
\text { none extends more than } 3 \text { inches into included timber. }{ }^{2}\end{array}$ \\
\hline \multicolumn{2}{|l|}{ Unsound surface defects } & $\begin{array}{l}\text { Same requirements as for sound defects if they extend } \\
\text { into included timber. }{ }^{2} \text { No limit if they do not. }\end{array}$ \\
\hline & Sound & No requirements. \\
\hline End defects & Unsound & $\begin{array}{l}\text { None allowed; log must be sound internally, but will ad- } \\
\text { mit one shake not to exceed one-fourth the scaling di- } \\
\text { ameter and will admit a longitudinal split not extending } \\
\text { more than } 5 \text { inches into the contained timber. }\end{array}$ \\
\hline
\end{tabular}

'These specifications are minimum for the class. If, from a group of logs, factory logs are selected first, thus leaving only non-factory logs from which to select construction logs, then the quality range of the construction logs so selected is limited, and the class may be considered a grade. If selection of construction logs is given first priority, then it may be necessary to subdivide the class into grades.

2Included timber is always square, and dimension is judged from small end. 
Softwood species were graded according to the following specifications:

\section{LOG GRADES FOR SOFTWOOD LOGS}

\section{Grade 1}

1. Logs must be 16 inches or larger, 10 feet or longer, and with deduction for defect not over 30 percent of gross scale.

2. Logs must be at least 75 percent clear on each of three faces.

3. All knots outside clear cutting must be sound and not over $2-1 / 2$ inches in diameter.

\section{Grade 2}

1. Logs must be 12 inches or larger, 10 feet or longer, and with a net scale after deduction for defect of at least 50 percent of the gross contents of the log.

2. Logs must be at least 50 percent clear on each of three faces or 75 percent clear on two faces.

\section{Grade 3}

Logs must be 6 inches or larger, 8 feet or longer, and with a net scale after deduction for defect of at least 50 percent of the gross contents of the log.

Note: (A) Diameters are diameter inside bark at small end of log.

(B) Percent clear refers to percent clear in one continuous section.

\section{PRINCIPAL TREE SPECIES GROUPS IN NEBRASKA ${ }^{2}$}

\section{Softwoods}

Ponderosa pine

Eastern redcedar Pinus ponderosa

Other softwoods

Rocky mountain juniper.. Juniperus scopulorum Hardwoods

White oak

Bur oak.

Quercus macrocarpa

White oak Quercus alba

Chinkapin oak Quercus muehlenbergii

Post oak Quercus stellata

Red oak

Northern red oak

Black oak. Quercus rubra Hickory

Shagbark hickory

Bitternut hickory.

Carya ovata Carya cordiformis

"The common and scientific names are based on: Little, Elbert L., Jr. Checklist of United States Trees ( $\mathrm{Na}$ tive and Naturalized). Agric. Handb. 541. Washington, DC: U.S. Department of Agriculture, Forest Service; 1979. $375 \mathrm{p}$.
Basswood

American basswood.

Soft maple

Red maple. Tilia americana

Silver maple

Acer rubrum

Boxelder. Acer saccharinum Elm

American elm Ulmus americana Siberian elm Ulmus pumila Slippery elm Ulmus rubra Rock elm Ulmus thomasii Green ash. Cottonwood

Eastern cottonwood Plains cottonwood.

Populus deltoides Populus deltoides var. occidentalis

Black willow Salix nigra

Hackberry. Celtis occidentalis

Black walnut

Other hardwoods

Honeylocust. Juglans nigra

Red mulberry

Gleditsia triacanthos

Paper birch.... Morus rubra

Texas buckeye Betula papyrifera Northern catalpa Kentucky coffeetree Osage orange Black locust.... White mulberry Fraxinus pennsylvanica

\section{METRIC EQUIVALENTS OF UNITS} USED IN THIS REPORT

1 acre $=4,046.86$ square meters or 0.405 hectare. 1,000 acres $=405$ hectares.

1 cubic foot $=0.0283$ cubic meter.

1 foot $=30.48$ centimeters or 0.3048 meter.

1 inch $=25.4$ millimeters, 2.54 centimeters, or 0.0254 meter.

\section{DEFINITION OF TERMS}

Basal area.--The area in square feet of the cross section at breast height of a single tree. When the basal area of all trees in a stand are summed, the result is usually expressed as square feet of basal area per acre.

Biomass.--The above-ground volume of all live trees (including bark and foliage). Biomass is made up of 5 components:

Growing-stock bole.--Biomass of a growing-stock tree from a 1-foot stump to a variable 4 -inch top.

Growing-stock tops and limbs.--Biomass of a growing-stock tree from a 1-foot stump minus the growing-stock bole.

Cull bole.--Biomass of a cull tree from a 1-foot stump to a variable 4 -inch top. 
Cull tops and limbs.--Biomass of a cull tree from a 1-foot stump minus the cull bole.

1- to 5-inch trees.--Biomass of all live trees from 1- to 5 -inches in diameter at breast height.

Commercial forest land.--Forest land producing or capable of producing crops of industrial wood and not withdrawn from timber utilization. (Note: Areas qualifying as commercial forest land are capable of producing more than 20 cubic feet per acre per year of annual growth when managed. Currently inaccessible and inoperable areas are included except when the areas involved are small and unlikely to become suitable for producing industrial wood in the foreseeable future.)

Commercial species.--Tree species presently or prospectively suitable for industrial wood products. (Note: Excludes species of typically small size, poor form, or inferior quality such as hophornbeam and hawthorn.)

County and municipal land.--Land owned by counties and local public agencies or municipalities, or land leased to these governmental units for 50 years or more.

Cull.--Portions of a tree that are unusable for industrial wood products because of rot, form, or other defect.

Diameter classes.--A classification of trees based on diameter outside bark, measured at breast height ( $41 / 2$ feet above the ground). (Note: d.b.h. is the common abbreviation for diameter at breast height. Two-inch diameter classes are commonly used in Forest Survey, with the even inch the approximate midpoint for a class. For example, the 6 -inch class includes trees 5.0 through 6.9 inches d.b.h. inclusive.)

Farm.--Any land from which $\$ 1,000$ or more of agricultural products were produced and sold during the year.

Farmer-owned lands.--Land owned by operators of farms. (Note: Excludes land leased by farm operators from nonfarm owners, such as railroad companies and States.)

Forest industry land.--Land owned by companies or individuals operating primary wood-using plants, except sawmills sawing less than 100,000 board feet annually.

Forest land.--Land at least 16.7 percent stocked by forest trees of any size, or formerly having had such tree cover, and not currently developed for nonforest use. (Note: Stocking is measured by comparison of basal area and/or number of trees, by age or size and spacing with specified standards.) The minimum area for classification of forest land is 1 acre. Roadside, streamside, and shelterbelt strips of timber must have a crown width at least 120 feet wide to qualify as forest land. Unimproved roads and trails, streams, or other bodies of water or clearings in forest areas shall be classes as forest if less than 120 feet wide. Also see definitions of land area, commercial forest land, noncommercial forest land, productive-reserved forest land, stocking, unproductive forest land, nonforest land, and water.

Forest trees.--Woody plants having a well-developed stem and usually more than 12 feet in height at maturity.

Forest types.--A classification of forest land based upon the species forming a plurality of live tree stocking. Major forest types in Nebraska are:

Ponderosa pine.--Forests in which ponderosa pine comprises a plurality of the stocking.

Eastern redcedar-hardwood.--Forests in which hardwoods comprise a plurality of the stocking but in which eastern redcedar comprises 25 percent or more of the stocking. Found on dry uplands, usually abandoned pastures or fields.

Oak-hickory.--Forests in which upland oaks (white, black) or hickory, singly or in combination, comprise a plurality of the stocking, except for stands classed as eastern redcedar-hardwood or as bur oak. Occurs on a variety of soils.

Bur oak.--Forests in which bur oak comprises a majority of the stocking. Occurs on dry uplands and ridges.

Elm-ash-cottonwood.--Lowland forest in which elm, ash, cottonwood, and willow, singly or in combination, comprise a plurality of the stocking, except for those in which cottonwood or willow comprise a majority of the stocking. Found on first or second bottoms of major streams.

Cottonwood.--Forests in which cottonwood comprises a majority of the stocking.

Lowland plains hardwoods.--Forests in which black walnut, hackberry, bur oak, soft maple, and boxelder, singly or in combination, comprise a plurality of the stocking. Commonly found in coves and bottomlands.

Gross area.--The entire area of land and water as determined by the Bureau of the Census, 1970.

Growing-stock trees.--Live trees of commercial species, excluding rough and rotten trees.

Growing-stock volume.--Net volume in cubic feet of growing-stock trees 5 inches d.b.h. and over, from a 1 -foot stump to a minimum 4 inch top diameter outside bark of the central stem or to the point where the central stem breaks into limbs. Cubic feet can be converted to standard cords by dividing by 79. One standard cord is 128 cubic feet of stacked wood, including bark and air.

Hardwoods.--Dicotyledonous trees, usually broadleaved and deciduous.

Idle farmland.--Includes former cropland, orchards, improved pastures, and farm sites not tended within the past 2 years and presently less than 16.7 percent stocked with trees. 
Improved pasture.--Land currently improved for grazing by cultivating, seeding, irrigating, or clearing of trees or brush and less than 16.7 percent stocked with live trees.

Indian land.--All lands held in trust by the United States for individual Indians or tribes, or all lands, titles to which are held by individual Indians or tribes, subject to Federal restrictions against alienation.

Land area.--A. Bureau of the Census.--The area of dry land and land temporarily or partly covered by water such as marshes, swamps, and river flood plains (omitting tidal flats below mean high tide); streams, sloughs, estuaries, and canals less than one-eighth of a statute mile wide; and lakes, reservoirs, and ponds less than 40 acres in area.

B. Forest Inventory and Analysis.--The same as the Bureau of the Census, except minimum width of streams, etc., is 120 feet and minimum size of lakes, etc., is 1 acre.

Live trees.--Growing-stock, rough, and rotten trees 1 inch d.b.h. and larger.

Log grades.--A classification of logs based on external characteristics as indicators of quality or value. (See Appendix for specific grading factors used.)

Logging residues.--The unused growing stock portions of trees cut or killed by logging.

Maintained road.--Any road, hard-topped or other surfaces, that is plowed or graded at least once a year. Includes rights-of-way that are cut or treated to limit herbaceous growth.

Marsh.--Nonforest land that characteristically supports low, generally herbaceous or shrubby vegetation and that is intermittently covered with water.

Merchantable.--Refers to a pulpwood or saw log section that meets pulpwood or saw log specifications, respectively.

Miscellaneous federal land.--Federal land other than National Forest and Indian land.

Miscellaneous private land.--Privately owned land other than forest-industry and farmer-owned land.

Mortality.-- The volume of sound wood in growingstock and sawtimber trees that die annually.

National Forest land.--Federal land that has been legally designated as National forest or purchase units, and other land administered by the USDA Forest Service.

Net annual growth of growing stock.--The annual change in volume of sound wood in live sawtimber and poletimber trees and the total volume of trees entering these classes through ingrowth, less volume losses resulting from natural causes.

Net annual growth of sawtimber.--The annual change in the volume of live sawtimber trees and the total volume of trees reaching sawtimber size, less volume losses resulting from natural causes.
Net volume.--Gross volume less deductions for rot, sweep, or other defect affecting use for timber products.

Noncommercial forest land.--(a) Unproductive forest land and (b) productive-reserved forest land.

Noncommercial species.--Tree species of typically small size, poor form, or inferior quality that normally do not develop into trees suitable for industrial wood products.

Nonforest land.--Land that has never supported forests, and land formerly forested where use for timber management is precluded by development for other uses. (Note: Includes areas used for crops, improved pasture, residential areas, city parks, improved roads of any width and adjoining clearings, powerline clearings of any width, and 1- to 40 -acre areas of water classified by the Bureau of the Census as land. If intermingled in forest areas, unimproved roads and nonforest strips must be more than 120 feet wide and more than 1 acre to qualify as nonforest land.)

a. Nonforest land without trees.--Nonforest land with no live trees present.

b. Nonforest land with trees.--Nonforest land with one or more trees per acre at least 5 inches d.b.h.

Nonstocked land.--Commercial forest land less than 16.7 percent stocked with growing-stock trees.

Other removals.--Growing-stock trees removed but not utilized for products, or trees left standing but "removed" from the commercial forest land classification by land use change. Examples are removals from cultural operations such as timber stand improvement work, land clearing, and changes in land use.

Ownership.--Property owned by one owner, regardless of the number of parcels in a specified area.

Ownership size class.--The amount of commercial forest land owned by one owner, regardless of the number of parcels.

Owner tenure.--The length of time a property has been held by the owner.

Physiographic class.--A measure of soil and water conditions that affect tree growth on a site. The physiographic classes are:

Xeric sites.--Very dry soils where excessive drainage seriously limits both growth and species occurrence. Example: sandy jack pine plains.

Xeromesic sites.--Moderately dry soils where excessive drainage limits growth and species occurrence to some extent. Example: dry oak ridge.

Mesic sites.--Deep, well-drained soils. Growth and species occurrence are limited only by climate.

Hydromesic sites.--Moderately wet soils where insufficient drainage or infrequent flooding limits growth and species occurrence to some extent. Example: better drained bottomland hardwood sites. 
Hydric sites.--Very wet sites where excess water seriously limits both growth and species occurrence. Example: frequently flooded river bottoms and spruce bogs.

Plant residues.-- Wood and bark materials generated at manufacturing plants during production of other products.

Poletimber stands.--(See stand-size class.)

Poletimber trees.--Growing-stock trees of commercial species at least 5 inches d.b.h. but smaller than sawtimber size.

Productive-reserved forest land.--Forest land sufficiently productive to qualify as commercial forest land but withdrawn from timber utilization through statute, administration regulation, designation, or exclusive use for Christmas tree production, as indicated by annual shearing.

Productive-deferred.--Forest land sufficiently productive to qualify as commercial forest land but presently withdrawn from timber utilization because it is being considered for possible inclusion into the Wilderness system.

Rotten trees.--Live trees of commercial species that do not contain at least one 12 -foot saw log or two saw logs 8 feet or longer, now or prospectively, because they do not meet regional specifications for freedom from defect primarily because of rot; that is, when more than 50 percent of extra cull volume in a tree is rotten.

Rough trees.--(a) Live trees of commercial species that do not contain at least one merchantable 12 foot saw $\log$ or two saw logs 8 feet or longer, now or prospectively, because they do not meet regional specifications for freedom from defect primarily because of roughness or poor form, and (b) all live trees of noncommercial species.

Roundwood products.--Logs, bolts, or other round sections (including chips from roundwood) cut from trees for industrial or consumer uses. (Note: Includes saw logs, veneer logs and bolts; cooperage logs and bolts; pulpwood, fuelwood; piling; poles; posts; hewn ties; mine timbers; and various other round, split, or hewn products.

Salvable dead trees.--Standing or down dead trees considered merchantable by regional standards.

Saplings.--Live trees 1 to 5 inches d.b.h.

Sapling-seedling stands.--(See stand-size class.)

Saw log.--A log meeting minimum standards of diameter, length, and defect, including logs at least 8 feet long, sound and straight and with a minimum diameter outside bark (d.o.b.) for softwoods of 7 inches (9 inches for hardwoods) or other combinations of size and defect specified by regional standards.

Saw log portion.--That part of the bole of sawtimber trees between the stump and the saw log top.
Saw $\log$ top.--The point on the bole of sawtimber trees above which a saw $\log$ cannot be produced. The minimum saw log top is 7 inches d.o.b. for softwoods and 9 inches d.o.b. for hardwoods.

Sawtimber stands.--(See stand-size class.)

Sawtimber trees.--Growing-stock trees of commercial species containing at least a 12 -foot saw log or two noncontiguous saw $\operatorname{logs} 8$ feet or longer, and meeting regional specifications for freedom from defect. Softwoods must be at least 9 inches d.b.h. Hardwoods must be at least 11 inches d.b.h.

Saw timber volume.--Net volume of the saw log portion of live sawtimber in board feet, International $1 / 4$-inch rule, from stump to a minimum 7 inches top diameter outside bark (d.o.b.) for softwoods and a minimum 9 inches top d.o.b. for hardwoods.

Seedlings.--Live trees less than 1 inch d.b.h. that are expected to survive. Only softwood seedlings more than 6 inches tall and hardwood seedlings more than 1 foot tall are counted.

Short-log (rough tree).--Sawtimber-size trees of commercial species that contain at least one merchantable 8 - to 11 -foot saw log but not a 12 -foot saw $\log$.

Site class.--A classification of forest land in terms of inherent capacity to grow crops of industrial wood based on fully stocked natural stands.

Site index.--An expression of forest site quality based on the height of a free-growing dominant or codominant tree of a representative species in the forest type at age 50 .

Softwoods.--Coniferous trees, usually evergreen, having needles or scale-like leaves.

Stand.--A growth of trees on a minimum of 1 acre of forest land that is stocked by forest trees of any size.

Stand-age class.--Age of the main stand. Main stand refers to trees of the dominant forest type and stand-size class.

Stand-area class.--The extent of a continuous forested area of the same forest type, stand-size class, and stand-density class.

Stand-size class.--A classification of forest land based on the size class of growing-stock trees on the area; that is, sawtimber, poletimber, or seedlings and saplings.

a. Sawtimber stands.--Stands at least 16.7 percent stocked with growing-stock trees, with half or more of total stocking in sawtimber and/or poletimber trees, and with sawtimber stocking at least equal to poletimber stocking.

b. Poletimber stands.--Stands at least 16.7 percent stocked with growing-stock trees of which half or more of this stocking is in poletimber and/or sawtimber trees, and with poletimber stocking exceeding that of sawtimber.

c. Sapling-seeding stands.--Stands at least 16.7 percent stocked with growing-stock trees of which 
more than half of the stocking is saplings and/or seedlings.

d. Nonstocked stands.--Stands in which stocking of growing-stock trees is less than 16.7 percent.

State land.--Land either owned by States or leased to them, for 50 years more.

Stocking.--The degree of occupancy of land by trees, measured by basal area and/or the number of trees in a stand by size or age and spacing, compared to the basal area and/or number of trees required to fully utilize the growth potential of the land; that is, the stocking standard.

A stocking percent of 100 indicates full utilization of the site and is equivalent to 80 square feet of basal area per acre in trees 5 inches d.b.h. and larger. In a stand of trees less than 5 inches d.b.h., a stocking percent of 100 would indicate that the present number of trees is sufficient to produce 80 square feet of basal area per acre when the trees reach 5 inches d.b.h.

Stands are grouped into the following stocking classes:

Overstocked stands.--Stands in which stocking of trees is 134.0 percent or more.

Fully-stocked stands.--Stands in which stocking of trees is from 101.0 to 133.9 percent.

Medium-stocked stands.--Stands in which stocking of trees is from 61.0 to 100.9 percent.

Poorly-stocked stands.--Stands in which stocking of trees is from 16.7 to 60.9 percent.

Nonstocked areas.--Commercial forest land on which stocking of trees is less than 16.7 percent.

Timber removals from growing stock.--The volume of sound wood in growing-stock trees removed annually for forest products (including roundwood products and logging residues) and for other removals.

Timber removals from sawtimber.--The net board-foot volume of live sawtimber trees removed for forest products annually (including roundwood products and logging residues) and for other removals.

Timber products output.--All timber products cut from roundwood and byproducts of wood manufacturing plants. Roundwood products include logs, bolts, or other round sections cut from growingstock trees, cull trees, salvable dead trees, trees on nonforest land, noncommercial species, sapling-size trees, and limbwood. Byproducts from primary manufacturing plants include slabs, edging, trimmings, miscuts, sawdust, shavings, veneer cores and clippings, and screenings of pulpmills that are used as pulpwood chips or other products.

Tree biomass.--The total aboveground weight (including the bark) of all trees from 1 to 5 inches in d.b.h., and the total aboveground weight (including the bark) from a 1-foot stump for trees more than 5 inches in diameter.

Tree size class.--A classification of trees based on diameter at breast height, including sawtimber trees, poletimber trees, saplings, and seedlings.

Unproductive forest land.--Forest land incapable of producing 20 cubic feet per acre of annual growth or of yielding crops of industrial wood under natural conditions because of adverse site conditions. (Note: Adverse conditions include shallow soil, dry climate, poor drainage, high elevation, steepness, and rockiness.)

Upper stem portion.--That part of the bole of sawtimber trees above the saw log top to a minimum top diameter of 4 inches outside bark or to the point where the central stem breaks into limbs.

Urban and other areas.--Areas within the legal boundaries of cities and towns; suburban areas developed for residential, industrial, or recreational purposes; schoolyards, cemeteries, roads; railroads; airports; beaches; powerlines; and other rights-ofway; or other nonforest land not included in any other specified land use class.

Water.--(a) Bureau of the Census.--Permanent inland water surfaces, such as lakes, reservoirs, and ponds at least 40 acres in area; and streams, sloughs, estuaries, and canals at least one-eighth of a statute mile wide.

(b) Noncensus.--Permanent inland water surfaces, such as lakes, reservoirs, and ponds from 1 to 39.9 acres in area; and streams, sloughs, estuaries, and canals from 120 feet to one-eighth of a statute mile wide.

Windbreak.--A group of trees less than 120 feet wide used for protecting soil, cropfields, and buildings.

Wooded pasture.--Improved pasture with more than 16.7 percent stocking in live trees but less than 25 percent stocking in growing-stock trees. Area is currently improved for grazing or there is other evidence of grazing.

Wooded strip.--An acre of more of natural continuous forest land that would otherwise meet survey standards for commercial forest land except that it is less than 120 feet wide. 
Table 1.--Area of land by land class, Nebraska $\stackrel{1 /}{ }, 1955$ and 1983

$$
\text { (In thousand acres) }
$$

\begin{tabular}{|c|c|c|}
\hline Land class & 1955 & 1983 \\
\hline \multicolumn{3}{|l|}{$\begin{array}{l}\text { Forest land: } \\
\text { Commercial forest land }\end{array}$} \\
\hline $\begin{array}{l}\text { Ponderosa pine } \\
\text { Eastern redcedar-hardwood } \\
\text { 0ak-hickory } \\
\text { Bur oak } \\
\text { Elm-ash-cottonwood } \\
\text { Cottonwood } \\
\text { Lowland plains hardwoods } \\
\text { Nonstocked }\end{array}$ & $\begin{array}{r}147.2 \\
12.5 \\
28.3 \\
45.1 \\
109.5 \\
83.3 \\
68.6 \\
220.2 \\
\end{array}$ & $\begin{array}{r}146.1 \\
42.2 \\
24.7 \\
37.4 \\
122.0 \\
82.8 \\
76.5 \\
6.1 \\
\end{array}$ \\
\hline \multirow{2}{*}{$\begin{array}{l}\text { Subtotal } \\
\text { Noncommercial forest land: } \\
\text { Unproductive } \\
\text { Productive-reserved }\end{array}$} & 714.7 & 537.8 \\
\hline & $\begin{array}{r}174.8 \\
13.8 \\
\end{array}$ & $\begin{array}{r}162.3 \\
18.2 \\
\end{array}$ \\
\hline \multirow{2}{*}{$\begin{array}{l}\text { Subtotal } \\
\text { Total }\end{array}$} & 188.6 & 180.5 \\
\hline & 903.3 & 718.3 \\
\hline Nonforest land: & $48,161.0$ & $48,333.8$ \\
\hline A11 land & $49,064.3^{21}$ & $49,052.13 /$ \\
\hline \multicolumn{3}{|c|}{$\begin{array}{l}\text { 1/ Figures have been adjusted from those published after the } \\
5 \text { survey to conform to } 1983 \text { areas because of changes in surve } \\
\text { initions and procedures. } \\
2 / 1950 \text {, Bureau of Census. } \\
3 / 1980 \text {, Bureau of Census. }\end{array}$} \\
\hline
\end{tabular}


Table 2.--Area by land class and Forest Survey Unit, Nebraska, 1983

(In thousand acres)

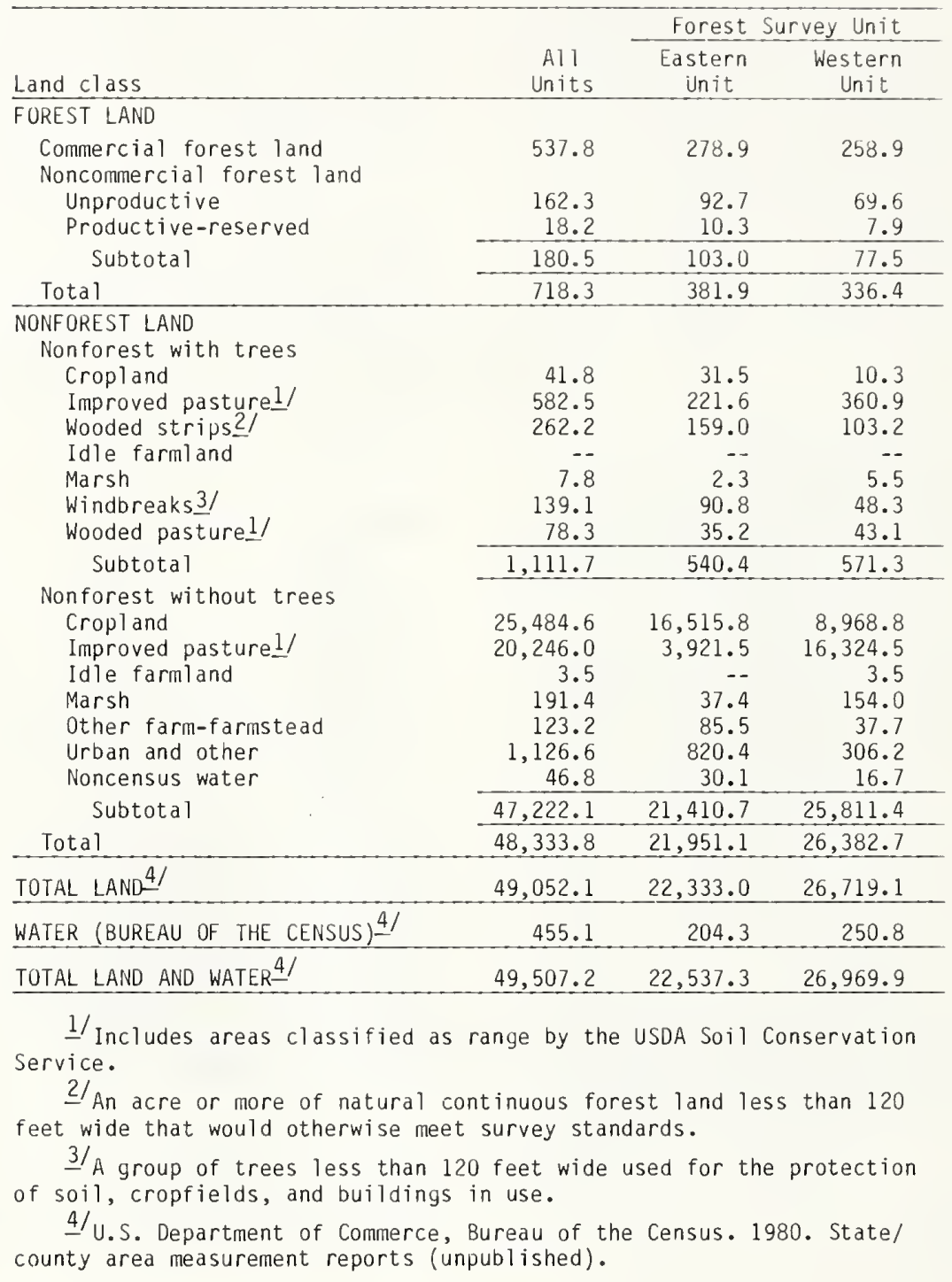




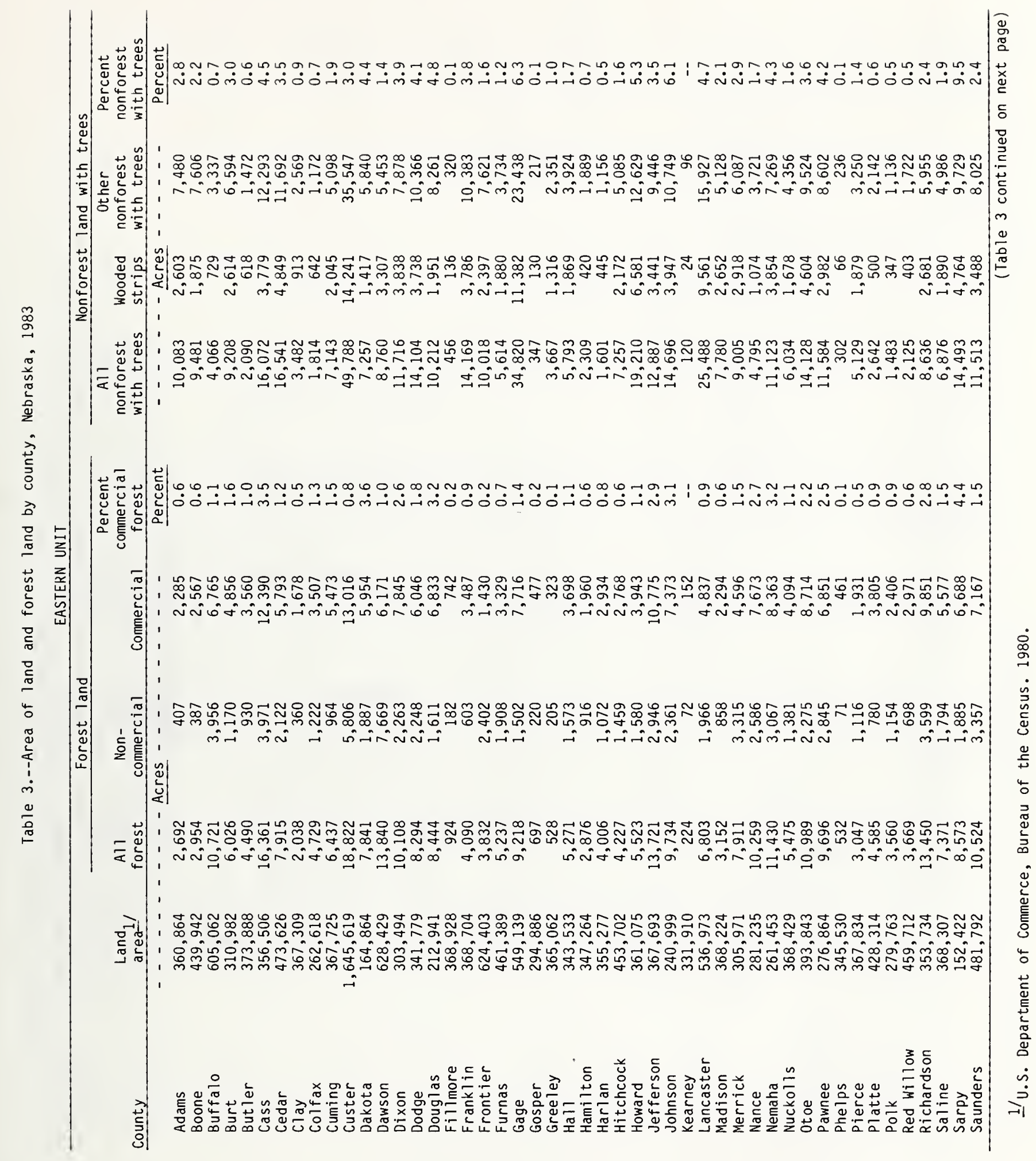




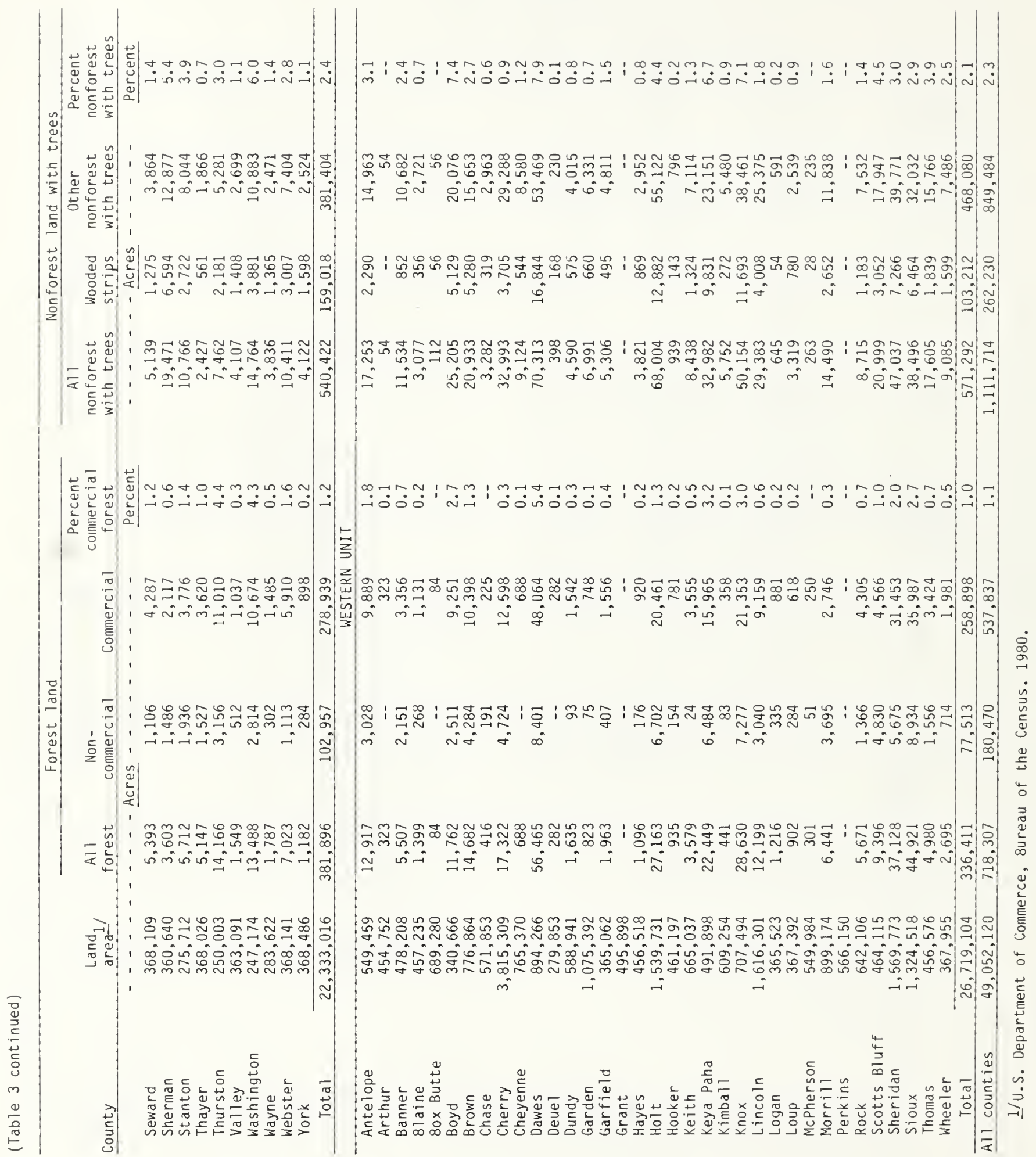



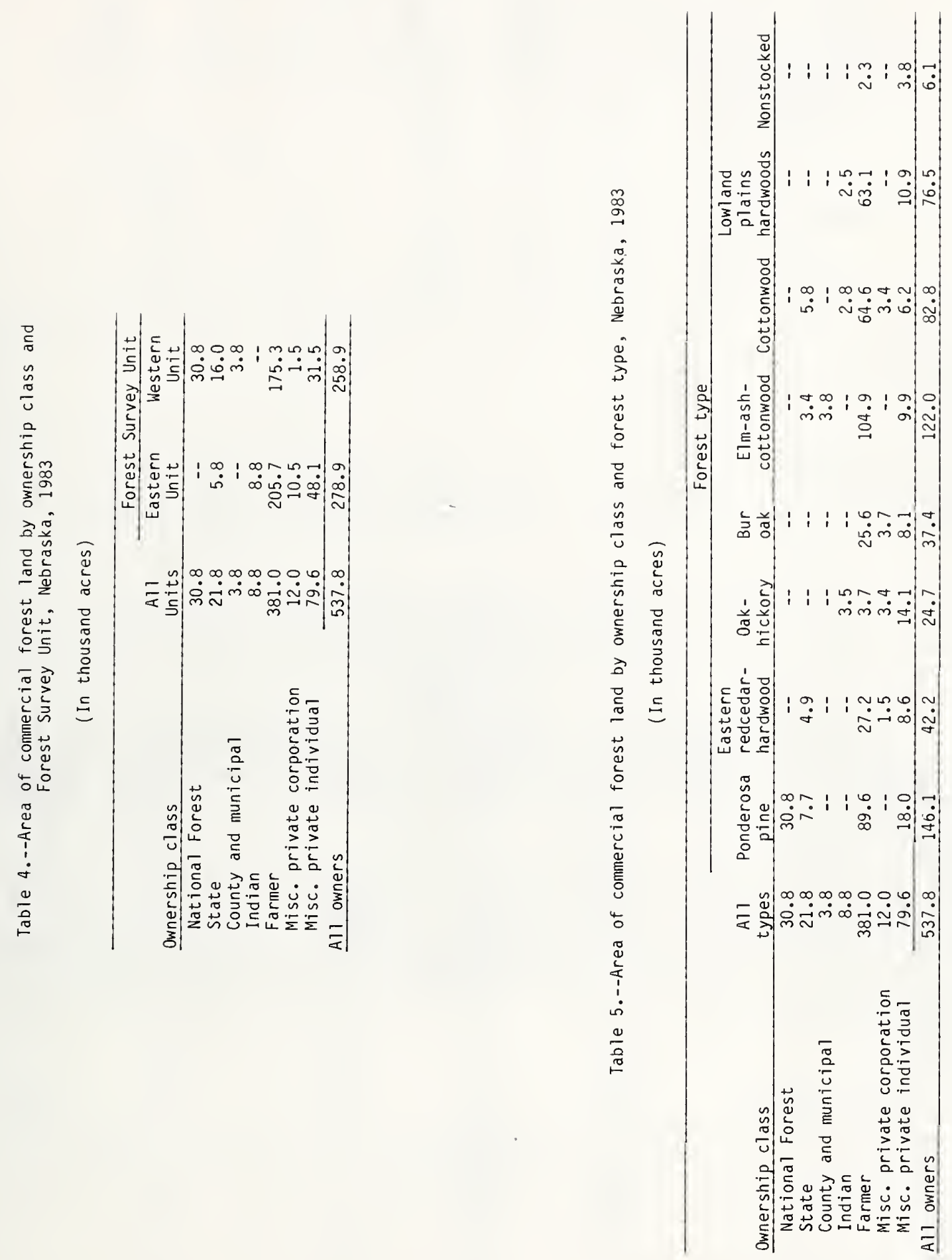
Table 6.--Area of commercial forest land by ownership class and site class, Nebraska, 1983

(In thousand acres)

\begin{tabular}{lrrrrr}
\hline & All & \multicolumn{2}{c}{ Site class (cubic feet of growth/acre/year) } \\
\cline { 3 - 6 } Ownership class & classes & $119+$ & $85-119$ & $50-84$ & $20-49$ \\
\hline National Forest & 30.8 & 2.7 & 16.0 & 9.0 & 3.1 \\
State & 21.8 & -- & 7.7 & -- & 14.1 \\
County and municipal & 3.8 & -- & -- & -- & 3.8 \\
Indian & 8.8 & -- & -- & 3.5 & 5.3 \\
Farmer & 381.0 & 9.1 & 55.6 & 87.2 & 229.1 \\
Misc. private corporation & 12.0 & -- & -- & 3.4 & 8.6 \\
Misc. private individual & 79.6 & -- & 5.9 & 23.1 & 50.6 \\
All owners & 537.8 & 11.8 & 85.2 & 126.2 & 314.6 \\
\hline
\end{tabular}

Table 7.--Area of commercial forest land by ownership class and ownership size class, Nebraska, 1983

(In thousand acres)

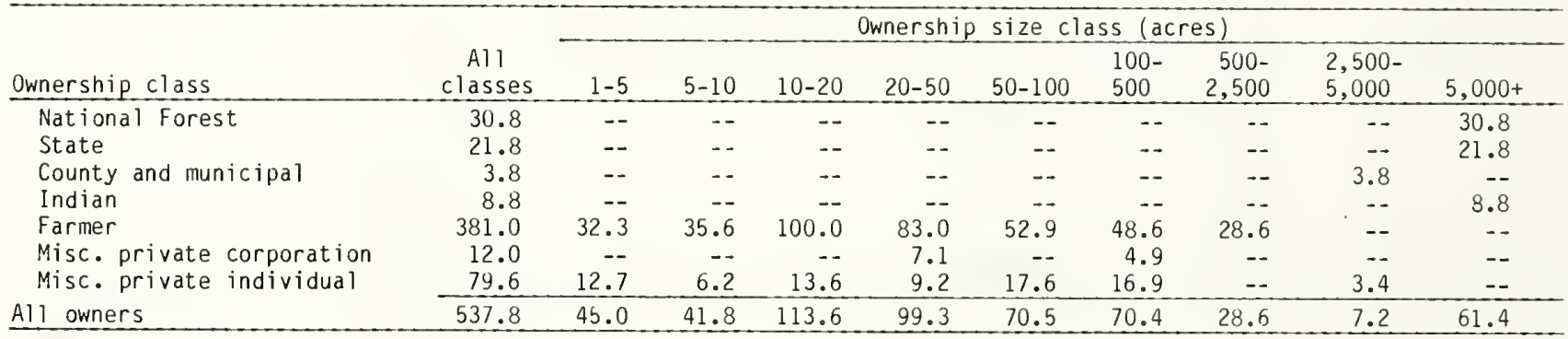

Table 8.--Area of commercial forest land by ownership class, stand-size class, and Forest Survey Unit, Nebraska, 1983

(In thousand acres)

ALL UNITS

\begin{tabular}{|c|c|c|c|c|c|}
\hline \multirow[b]{2}{*}{ Ownership class } & \multirow[b]{2}{*}{$\begin{array}{l}\text { Al } 1 \\
\text { stands }\end{array}$} & \multicolumn{4}{|c|}{ Stand-size class } \\
\hline & & $\begin{array}{c}\text { Sawt imber } \\
\text { stands }\end{array}$ & $\begin{array}{c}\text { Poletimber } \\
\text { stands }\end{array}$ & $\begin{array}{c}\text { Sapling and } \\
\text { seedling stands }\end{array}$ & $\begin{array}{c}\text { Nonstocked } \\
\text { areas }\end{array}$ \\
\hline National Forest & 30.8 & 27.7 & - & 3.1 & - \\
\hline State & 21.8 & 18.4 & -- & 3.4 & $-\rightarrow$ \\
\hline County and municipal & 3.8 & 3.8 & -- & -- & -- \\
\hline Indian & 8.8 & -- & 6.0 & 2.8 & -- \\
\hline Farmer & 381.0 & 261.0 & 57.5 & 60.2 & 2.3 \\
\hline Misc. private corporation & 12.0 & 8.6 & 3.4 & -. & $\ldots$ \\
\hline Misc. private individual & 79.6 & 50.4 & 22.0 & 3.4 & 3.8 \\
\hline All owners & 537.8 & 369.9 & 88.9 & 72.9 & 6.1 \\
\hline \multicolumn{6}{|c|}{ EASTERN UNIT } \\
\hline National Forest & - & - & - & -- & -- \\
\hline State & 5.8 & 5.8 & -- & -- & -- \\
\hline County and municipal & - & -- & -- & -- & - \\
\hline Indian & 8.8 & -- & 6.0 & 2.8 & -- \\
\hline Farmer & 205.7 & 128.7 & 28.4 & 48.6 & -- \\
\hline Misc. private corporation & 10.5 & 7.1 & 3.4 & -- & -- \\
\hline Misc. private individual & 48.1 & 27.6 & 13.3 & 3.4 & 3.8 \\
\hline A11 owners & 278.9 & 169.2 & 51.1 & 54.8 & 3.8 \\
\hline \multicolumn{6}{|c|}{ WESTERN UNIT } \\
\hline National Forest & 30.8 & 27.7 & - & 3.1 & -- \\
\hline State & 16.0 & 12.6 & -- & 3.4 & - \\
\hline County and municipal & 3.8 & 3.8 & -- & -- & -- \\
\hline Indian & -- & $\cdots$ & -- & -- & -- \\
\hline Farmer & 175.3 & 132.3 & 29.1 & 11.6 & 2.3 \\
\hline Misc. private corporation & 1.5 & 1.5 & -- & -- & - \\
\hline Misc. private individual & 31.5 & 22.8 & 8.7 & -- & $\therefore$ \\
\hline All owners & 258.9 & 200.7 & 37.8 & 18.1 & 2.3 \\
\hline
\end{tabular}


Table 9.--Area of commercial forest land by ownership class and area-condition class, Nebraska, 1983

(In thousand acres)

\begin{tabular}{|c|c|c|c|c|c|}
\hline \multirow[b]{2}{*}{ Ownership class } & \multirow[b]{2}{*}{$\begin{array}{c}\text { All } \\
\text { classes }\end{array}$} & \multicolumn{4}{|c|}{ Area-condition class } \\
\hline & & 70 & 60 & 50 & $\begin{array}{l}40 \text { or } \\
\text { better }\end{array}$ \\
\hline National Forest & 30.8 & 5.9 & 17.7 & 7.2 & -- \\
\hline State & 21.8 & 6.3 & 12.6 & 2.9 & -- \\
\hline County and municipal & 3.8 & 3.8 & -- & -- & -- \\
\hline Indian & 8.8 & 5.3 & 3.5 & _- & -. \\
\hline Farmer & 381.0 & 149.1 & 185.3 & 46.6 & $\ldots$ \\
\hline Misc. private corporation & 12.0 & 3.7 & 4.9 & 3.4 & -- \\
\hline Misc. private individual & 79.6 & 33.3 & 37.0 & 9.3 & -- \\
\hline All owners & 537.8 & 207.4 & 261.0 & 69.4 & -. \\
\hline
\end{tabular}

Table 10.--Area of commercial forest land by ownership class and stand-volume class, Nebraska, 1983

(In thousand acres)

\begin{tabular}{|c|c|c|c|c|}
\hline \multirow[b]{2}{*}{ Ownership class } & \multirow[b]{2}{*}{$\begin{array}{c}\text { All } \\
\text { classes } \\
\end{array}$} & \multicolumn{3}{|c|}{ Stand-volume class (board feet $1 /$ per acre) } \\
\hline & & $\begin{array}{c}\text { Less than } \\
1,500\end{array}$ & $\begin{array}{c}1,500 \text { to } \\
5,000\end{array}$ & $5,000+$ \\
\hline National Forest & 30.8 & 5.9 & 13.0 & 11.9 \\
\hline State & 21.8 & 3.4 & 7.8 & 10.6 \\
\hline County and municipal & 3.8 & 3.8 & - & $-\infty$ \\
\hline Indian & 8.8 & 8.8 & -- & -. \\
\hline Farmer & 381.0 & 146.2 & 181.4 & 53.4 \\
\hline Misc. private corporation & 12.0 & 8.6 & 3.4 & -- \\
\hline Misc. private individual & 79.6 & 43.3 & 36.3 & -- \\
\hline All owners & 537.8 & 220.0 & 241.9 & 75.9 \\
\hline
\end{tabular}

$\underline{1}$ International $1 / 4$-inch rule. 
Table 11.--Area of commercial forest land by county and forest type, Nebraska, 1983

(In acres)

EASTERN UNIT

\begin{tabular}{|c|c|c|c|c|c|c|c|c|c|}
\hline \multirow[b]{2}{*}{ County } & \multirow[b]{2}{*}{$\begin{array}{c}\text { Al1 } \\
\text { types }\end{array}$} & \multicolumn{8}{|c|}{ Forest type } \\
\hline & & $\begin{array}{l}\text { Ponderosa } \\
\text { pine }\end{array}$ & $\begin{array}{l}\text { Eastern } \\
\text { redcedar- } \\
\text { hardwood }\end{array}$ & $\begin{array}{c}\text { Oak- } \\
\text { hickory }\end{array}$ & $\begin{array}{l}\text { Bur } \\
\text { oak }\end{array}$ & $\begin{array}{c}\text { Elm-ash- } \\
\text { cottonwood }\end{array}$ & Cottonwood & $\begin{array}{c}\text { Lowland } \\
\text { plains } \\
\text { hardwoods }\end{array}$ & Nonstocked \\
\hline Adams & 2,285 & -- & 68 & 250 & 346 & 966 & 341 & 306 & 8 \\
\hline Boone & 2,567 & -- & 4 & 323 & 306 & 1,145 & 214 & 571 & 4 \\
\hline Buffalo & 6,765 & -- & 641 & 26 & 263 & 1,872 & 2,337 & 1,470 & 156 \\
\hline Burt & 4,856 & -- & 77 & 655 & 775 & 1,404 & 745 & 1,192 & 8 \\
\hline Butler & 3,560 & -- & 144 & 557 & 194 & 1,294 & 682 & 637 & 52 \\
\hline Cass & 12,390 & -- & 673 & 941 & 1,001 & 4,789 & 2,041 & 2,812 & 133 \\
\hline Cedar & 5,793 & -- & 74 & 192 & 1,104 & 1,805 & 328 & 2,274 & 16 \\
\hline Clay & 1,678 & -- & 40 & 303 & 211 & 611 & 360 & 149 & 4 \\
\hline Colfax & 3,507 & -- & 82 & 625 & 115 & 1,134 & 746 & 723 & 82 \\
\hline Cuming & 5,473 & -- & 362 & 574 & 416 & 2,108 & 1,027 & 950 & 36 \\
\hline Custer & 13,016 & -- & 357 & 724 & 647 & 5,151 & 1,680 & 4,273 & 184 \\
\hline Dakota. & 5,954 & -- & 164 & 442 & 1,251 & 1,990 & 694 & 1,365 & 48 \\
\hline Dawson & 6,171 & -- & 453 & 260 & 138 & 975 & 1,982 & 2,264 & 99 \\
\hline Dixon & 7,845 & -- & 43 & 246 & 1,872 & 2,437 & 240 & 2,996 & 11 \\
\hline Dodge & 6,046 & -- & 544 & 73 & 316 & 2,428 & 1,334 & 1,244 & 107 \\
\hline Dougl as & 6,833 & -- & 31 & 982 & 1,520 & 1,977 & 771 & 1,533 & 19 \\
\hline Fillmore & 742 & -- & 34 & 28 & 37 & 313 & 83 & 235 & 12 \\
\hline Frankl in & 3,487 & -- & 70 & 378 & 476 & 1,362 & 393 & 796 & 12 \\
\hline Frontier & 1,430 & -- & 20 & 172 & 200 & 632 & 155 & 243 & 8 \\
\hline Furnas & 3,329 & -- & 282 & 146 & 161 & 1,081 & 791 & 811 & 57 \\
\hline Gage & 7,716 & -- & 30 & 900 & 1,250 & 2,933 & 632 & 1,963 & 8 \\
\hline Gosper & 477 & -- & 16 & 45 & 16 & 163 & 84 & 137 & 16 \\
\hline Greeley & 323 & -- & 12 & -- & 12 & 115 & 36 & 136 & 12 \\
\hline $\mathrm{Hall}$ & 3,698 & -- & 396 & -- & 153 & 1,062 & 1,419 & 617 & 51 \\
\hline Hamilton & 1,960 & -- & 94 & 225 & 53 & 595 & 530 & 427 & 36 \\
\hline Harlan & 2,934 & -- & 89 & 445 & 89 & 976 & 623 & 623 & 89 \\
\hline Hitchcock & 2,768 & -- & 50 & 460 & 313 & 1,084 & 430 & 415 & 16 \\
\hline Howard & 3,943 & -- & 125 & 510 & 125 & 1,327 & 783 & 948 & 125 \\
\hline Jefferson & 10,775 & -- & 318 & 865 & 895 & 4,385 & 1,173 & 3,043 & 96 \\
\hline Johnson & 7,373 & -- & 305 & 731 & 490 & 2,966 & 1,387 & 1,397 & 97 \\
\hline Kearney & 152 & -- & 8 & -- & 8 & 56 & 24 & 48 & 8 \\
\hline Lancaster & 4,837 & -- & 249 & 585 & 293 & 2,005 & 861 & 777 & 67 \\
\hline Madison & 2,294 & -- & 66 & 355 & 66 & 762 & 482 & 497 & 66 \\
\hline Merrick & 4,596 & -- & 414 & 196 & 197 & 1,006 & 1,605 & 1,125 & 53 \\
\hline Nance & 7,673 & -- & 411 & 826 & 414 & 2,779 & 1,502 & 1,576 & 165 \\
\hline Nemaha & 8,363 & -- & 216 & 1,345 & 282 & 2,739 & 1,724 & 1,841 & 216 \\
\hline Nuckolls & 4,094 & -- & 361 & 233 & 253 & 1,443 & 983 & 770 & 51 \\
\hline Otoe & 8,714 & -- & 326 & 1,618 & 502 & 2,960 & 1,904 & 1,312 & 92 \\
\hline Pawnee & 6,851 & -- & 570 & 194 & 382 & 2,553 & 1,533 & 1,498 & $12:$ \\
\hline Phelps & 461 & -- & 22 & - & 153 & 143 & 33 & 110 & -- \\
\hline Pierce & 1,931 & -- & 82 & -- & 82 & 697 & 246 & 742 & 82 \\
\hline Platte & 3,805 & -- & 269 & 315 & 342 & 1,494 & 738 & 606 & 41 \\
\hline Polk & 2,406 & -- & 166 & 140 & 69 & 823 & 515 & 672 & 21 \\
\hline Red Willow & 2,971 & -- & 235 & 198 & 185 & 1,149 & 615 & 538 & 51 \\
\hline Richardson & 9,851 & -- & 291 & 1,530 & 291 & 3,267 & 2,097 & 2,084 & 291 \\
\hline Saline & 5,577 & -- & 325 & 597 & 380 & 2,111 & 988 & 1,093 & 83 \\
\hline Sarpy & 6,688 & -- & 91 & 416 & 1,753 & 1,877 & 552 & 1,972 & 27 \\
\hline
\end{tabular}




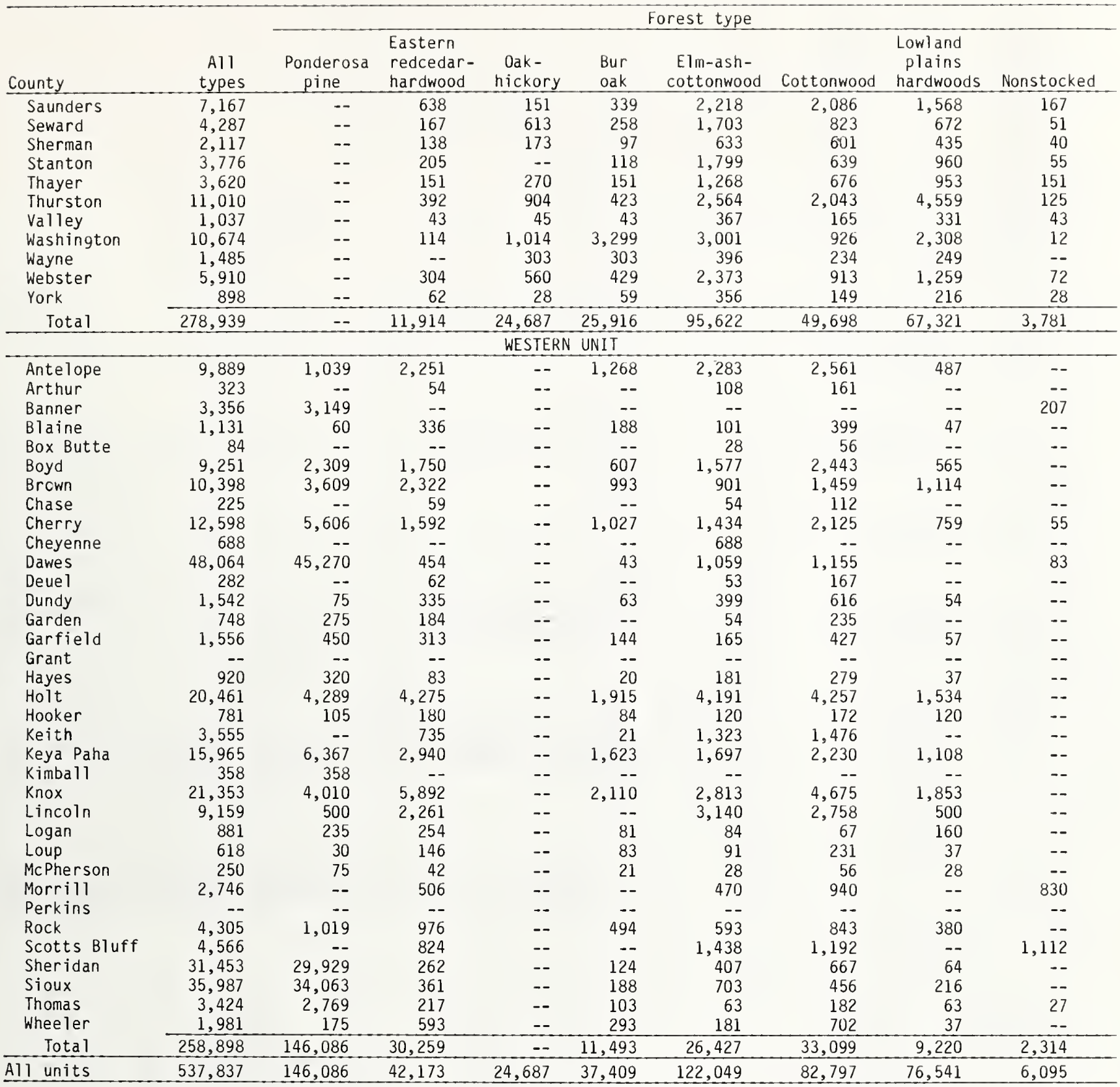




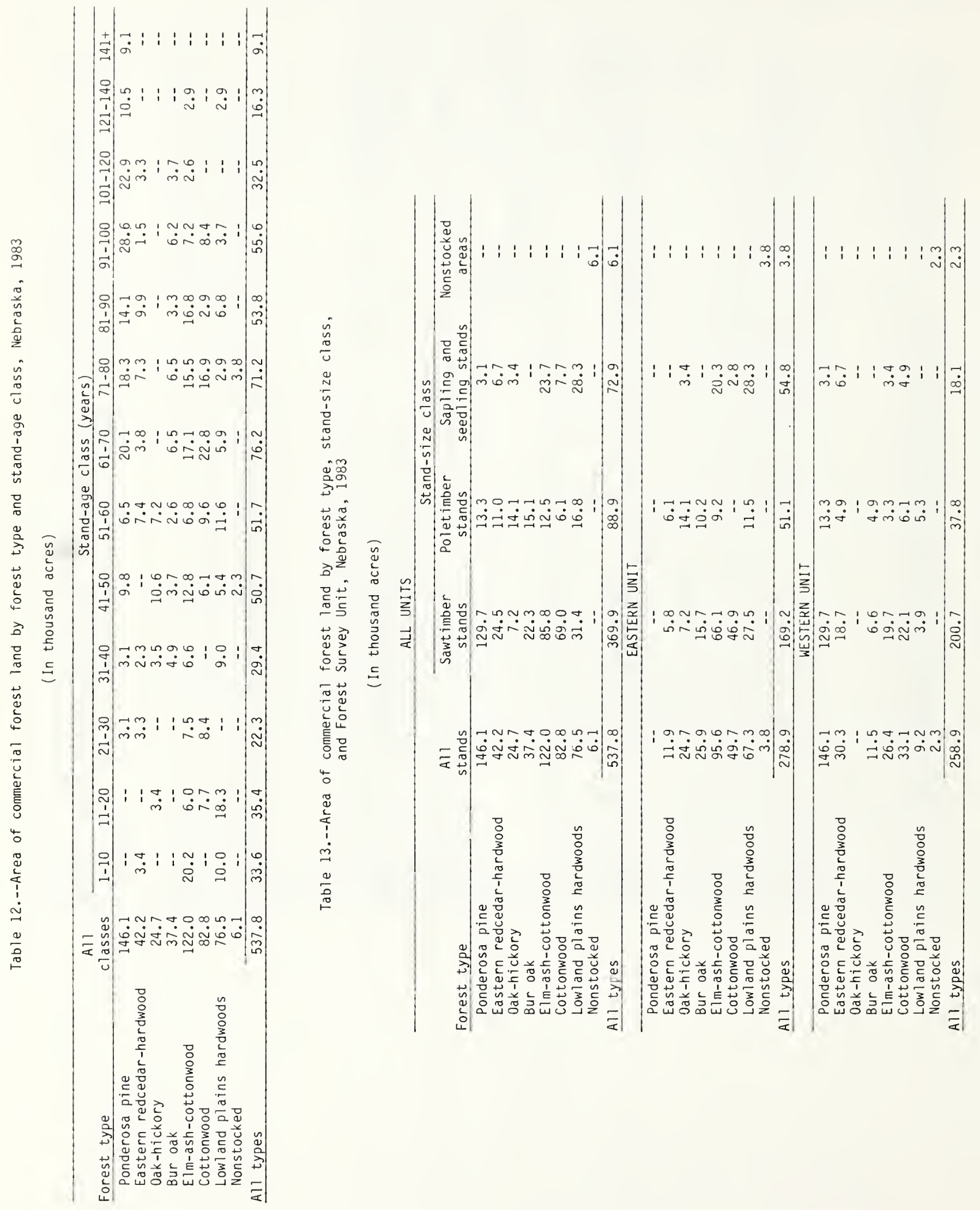


Table 14.--Area of commercial forest land by forest type, stand-size class, and site class, Nebraska, 1983

(In thousand acres)

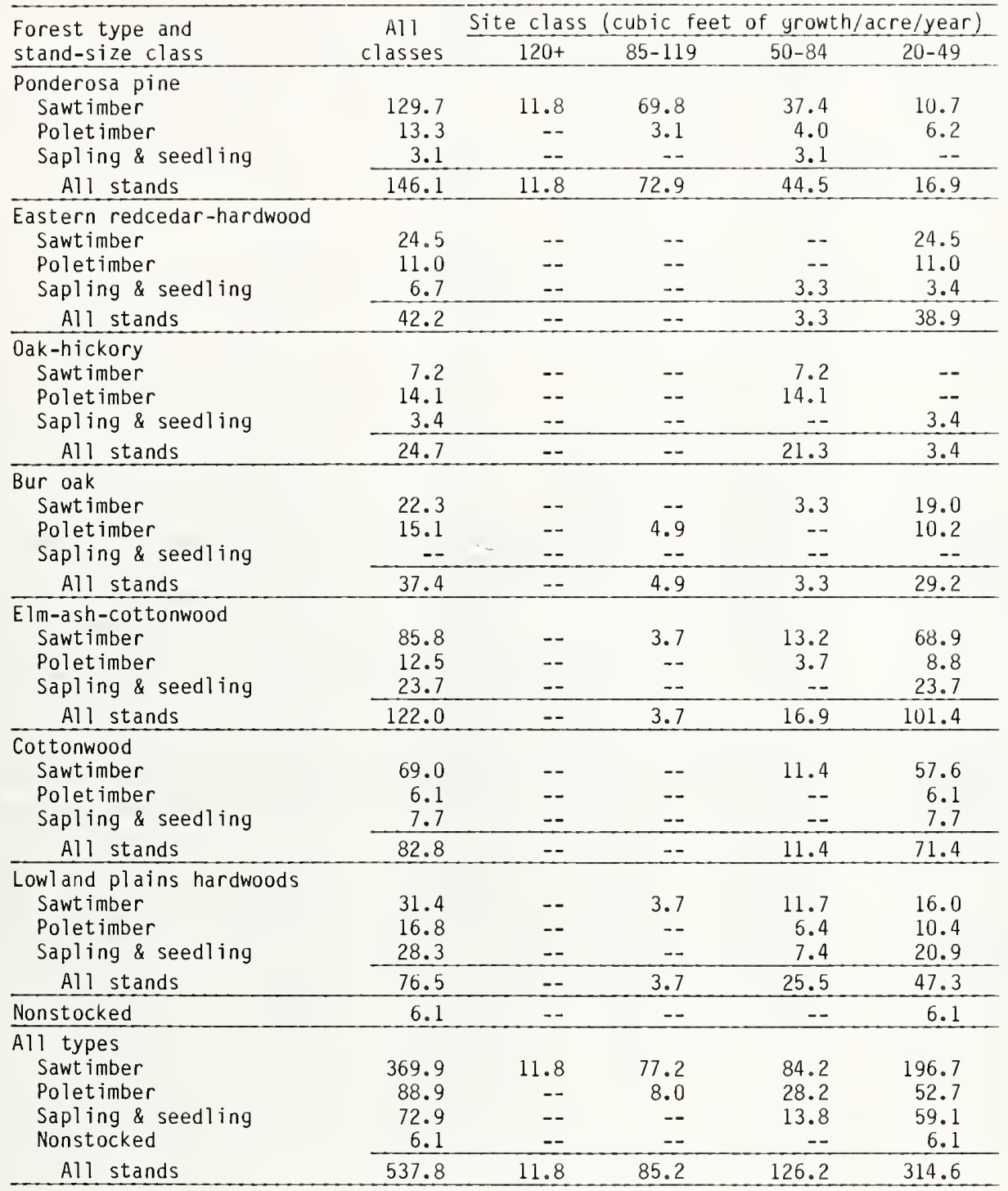


Table 15.--Area of commercial forest land by forest type, stand-size class, and stocking percent, Nebraska, 1983

(In thousand acres)

\begin{tabular}{|c|c|c|c|c|c|c|}
\hline \multirow[b]{2}{*}{$\begin{array}{l}\text { Forest type and } \\
\text { stand-size class }\end{array}$} & \multirow[b]{2}{*}{$\begin{array}{c}\text { All } \\
\text { classes }\end{array}$} & \multicolumn{5}{|c|}{ Stocking percent of growing-stock trees } \\
\hline & & $\begin{array}{l}\text { Less than } \\
16.7\end{array}$ & $16.7-60$ & $61-100$ & $101-133$ & $134+$ \\
\hline \\
\hline Sawt imber & 129.7 & -- & 36.8 & 72.9 & 20.0 & -- \\
\hline Poletimber & 13.3 & -- & 7.1 & 6.2 & -- & -- \\
\hline Sapling \& seedling & 3.1 & - & -- & -- & 3.1 & -- \\
\hline All stands & 146.1 & -- & 43.9 & 79.1 & 23.1 & -- \\
\hline \multicolumn{7}{|c|}{ Eastern redcedar-hardwood } \\
\hline Sawtimber & 24.5 & -- & 10.2 & 14.3 & -- & -- \\
\hline Poletimber & 11.0 & -- & 7.2 & 3.8 & -- & $-\infty$ \\
\hline Sapling \& seedling & 6.7 & $=-$ & 6.7 & $\ldots$ & $=$ & -- \\
\hline All stands & 42.2 & -- & 24.1 & 18.1 & -- & -- \\
\hline \multicolumn{7}{|l|}{ 0ak-hickory } \\
\hline Sawtimber & 7.2 & -- & -- & 3.7 & 3.5 & -- \\
\hline Poletimber & 14.1 & -- & -- & 10.7 & 3.4 & -- \\
\hline Sapling \& seedling & 3.4 & -- & 3.4 & - & -- & -- \\
\hline All stands & 24.7 & -- & 3.4 & 14.4 & 6.9 & -- \\
\hline \multicolumn{7}{|l|}{ Bur oak } \\
\hline Sawt imber & 22.3 & -- & 12.5 & 9.8 & -- & -- \\
\hline Poletimber & 15.1 & -- & -- & 15.1 & -- & -- \\
\hline Sapling \& seedling & -- & -- & -- & -- & - & -- \\
\hline All stands & 37.4 & $=-$ & 12.5 & 24.9 & -- & -- \\
\hline \multicolumn{7}{|l|}{ Elm-ash-cottonwood } \\
\hline Sawtimber & 85.8 & -- & 47.7 & 28.6 & 9.5 & -- \\
\hline Poletimber & 12.5 & -- & 5.9 & 6.6 & - & -- \\
\hline Sapling \& seedling & 23.7 & $\therefore$ & 17.4 & 3.4 & 2.9 & -- \\
\hline All stands & 122.0 & -- & 71.0 & 38.6 & 12.4 & -- \\
\hline \multicolumn{7}{|l|}{ Cottonwood } \\
\hline Sawtimber & 69.0 & -- & 12.7 & 38.1 & 12.4 & 5.8 \\
\hline Poletimber & 6.1 & -- & 3.7 & 2.4 & -- & -- \\
\hline Sapling \& seedling & 7.7 & -- & 2.8 & 4.9 & -- & -- \\
\hline All stands & 82.8 & $=-$ & 19.2 & 45.4 & 12.4 & 5.8 \\
\hline \multicolumn{7}{|c|}{ Lowl and plains hardwoods } \\
\hline Sawtimber & 31.4 & -- & 7.6 & 22.2 & 1.6 & -- \\
\hline Poletimber & 16.8 & -- & 10.0 & 6.8 & -- & -- \\
\hline Sapling \& seedling & 28.3 & -- & 15.6 & 12.7 & -- & -- \\
\hline All stands & 76.5 & -- & 33.2 & 41.7 & 1.6 & -- \\
\hline Nonstocked & 6.1 & 6.1 & -- & -- & $\because$ & -- \\
\hline \multicolumn{7}{|l|}{ All types } \\
\hline Sawt imber & 369.9 & -- & 127.5 & 189.6 & 47.0 & 5.8 \\
\hline Poletimber & 88.9 & -- & 33.9 & 51.6 & 3.4 & -- \\
\hline Sapling \& seedling & 72.9 & -- & 45.9 & 21.0 & 6.0 & -- \\
\hline Nonstocked & 6.1 & 6.1 & -- & $=$ & -- & -- \\
\hline All stands & 537.8 & 6.1 & 207.3 & 262.2 & 56.4 & 5.8 \\
\hline
\end{tabular}


Table 16.--Area of commercial forest land by forest type, site-index class, and Forest Survey Unit, Nebraska, 1983

(In thousand acres)

\begin{tabular}{|c|c|c|c|c|c|c|c|c|c|}
\hline \multirow[b]{2}{*}{ Forest type } & \multirow{2}{*}{$\begin{array}{c}\text { All } \\
\text { classes }\end{array}$} & \multicolumn{8}{|c|}{ Site-index class (feet) } \\
\hline & & $21-30$ & $31-40$ & $41-50$ & $51-60$ & $61-70$ & $71-80$ & $81-90$ & $91+$ \\
\hline $\begin{array}{l}\text { Ponderosa pine } \\
\text { Eastern redcedar-hardwood } \\
\text { Oak-hickory } \\
\text { Bur oak } \\
\text { Elm-ash-cottonwood } \\
\text { Cottonwood } \\
\text { Lowland plains hardwoods } \\
\text { Nonstocked }\end{array}$ & $\begin{array}{r}146.1 \\
42.2 \\
24.7 \\
37.4 \\
122.0 \\
82.8 \\
76.5 \\
6.1 \\
\end{array}$ & $\begin{array}{r}-- \\
10.2 \\
-- \\
-- \\
-- \\
-- \\
--\end{array}$ & $\begin{array}{r}3.1 \\
11.8 \\
-- \\
15.5 \\
22.3 \\
14.6 \\
11.5 \\
2.3 \\
\end{array}$ & $\begin{array}{r}28.8 \\
6.7 \\
-- \\
7.2 \\
40.1 \\
20.3 \\
19.0 \\
3.8 \\
\end{array}$ & $\begin{array}{r}29.5 \\
10.2 \\
10.3 \\
6.5 \\
28.0 \\
24.1 \\
16.8 \\
\ldots\end{array}$ & $\begin{array}{r}64.5 \\
3.3 \\
14.4 \\
-- \\
13.9 \\
18.6 \\
14.0 \\
-=\end{array}$ & $\begin{array}{r}14.4 \\
-- \\
-- \\
8.2 \\
14.0 \\
5.2 \\
15.2 \\
-.\end{array}$ & $\begin{array}{c}5.8 \\
-- \\
-- \\
-- \\
-- \\
-- \\
-- \\
\end{array}$ & $\begin{array}{l}-- \\
-- \\
-- \\
3.7 \\
-- \\
--\end{array}$ \\
\hline All types & 537.8 & 10.2 & 81.1 & 125.9 & 125.4 & 128.7 & 57.0 & 5.8 & 3.7 \\
\hline \multicolumn{10}{|c|}{ EASTERN UNIT } \\
\hline $\begin{array}{l}\text { Ponderosa pine } \\
\text { Eastern redcedar-hardwood } \\
\text { Oak-hickory } \\
\text { Bur oak } \\
\text { Elm-ash-cottonwood } \\
\text { Cottonwood } \\
\text { Lowland plains hardwoods } \\
\text { Nonstocked }\end{array}$ & $\begin{array}{r}-- \\
11.9 \\
24.7 \\
25.9 \\
95.6 \\
49.7 \\
67.3 \\
3.8 \\
\end{array}$ & $\begin{array}{l}-- \\
3.8 \\
-- \\
-- \\
-- \\
-- \\
-- \\
--\end{array}$ & $\begin{array}{r}-- \\
2.9 \\
-- \\
15.5 \\
11.8 \\
7.3 \\
11.5 \\
--\end{array}$ & $\begin{array}{r}-- \\
-- \\
-- \\
3.9 \\
32.8 \\
15.4 \\
13.7 \\
3.8\end{array}$ & $\begin{array}{r}5 .- \\
10.3 \\
6.5 \\
19.4 \\
15.5 \\
13.5 \\
---\end{array}$ & $\begin{array}{r}-- \\
-- \\
14.4 \\
-- \\
13.9 \\
6.3 \\
14.0 \\
--\end{array}$ & $\begin{array}{r}-- \\
-- \\
-- \\
-- \\
14.0 \\
5.2 \\
14.6 \\
--\end{array}$ & $\begin{array}{l}-- \\
-- \\
-- \\
-- \\
-- \\
-- \\
-- \\
--\end{array}$ & $\begin{array}{l}-- \\
-- \\
-- \\
3.7 \\
-- \\
-- \\
-\end{array}$ \\
\hline All types & 278.9 & 3.8 & 49.0 & 69.6 & 70.4 & 48.6 & 33.8 & $\because$ & 3.7 \\
\hline \multicolumn{10}{|c|}{ WESTERN UNIT } \\
\hline $\begin{array}{l}\text { Ponderosa pine } \\
\text { Eastern redcedar-hardwood } \\
\text { Oak-hickory } \\
\text { Bur oak } \\
\text { Elm-ash-cottonwood } \\
\text { Cottonwood } \\
\text { Lowl and plains hardwoods } \\
\text { Nonstocked }\end{array}$ & $\begin{array}{r}146.1 \\
30.3 \\
-- \\
11.5 \\
26.4 \\
33.1 \\
9.2 \\
2.3\end{array}$ & $\begin{array}{r}-- \\
6.4 \\
-- \\
-- \\
-- \\
-- \\
-- \\
--\end{array}$ & $\begin{array}{r}3.1 \\
8.9 \\
-- \\
-- \\
10.5 \\
7.3 \\
-- \\
2.3\end{array}$ & $\begin{array}{r}28.8 \\
6.7 \\
-- \\
3.3 \\
7.3 \\
4.9 \\
5.3 \\
-. \\
\end{array}$ & $\begin{array}{r}29.5 \\
5.0 \\
-- \\
-- \\
8.6 \\
8.6 \\
3.3 \\
\ldots\end{array}$ & $\begin{array}{r}64.5 \\
3.3 \\
-- \\
-- \\
-- \\
12.3 \\
-- \\
--\end{array}$ & $\begin{array}{r}14.4 \\
-- \\
-- \\
8.2 \\
-- \\
-- \\
0.6 \\
-- \\
\end{array}$ & $\begin{array}{c}5.8 \\
-- \\
-- \\
-- \\
-- \\
-- \\
-- \\
-- \\
\end{array}$ & $\begin{array}{l}-- \\
- \\
- \\
- \\
- \\
-- \\
-- \\
--\end{array}$ \\
\hline All types & 258.9 & 6.4 & 32.1 & 56.3 & 55.0 & 80.1 & 23.2 & 5.8 & $\because$ \\
\hline
\end{tabular}




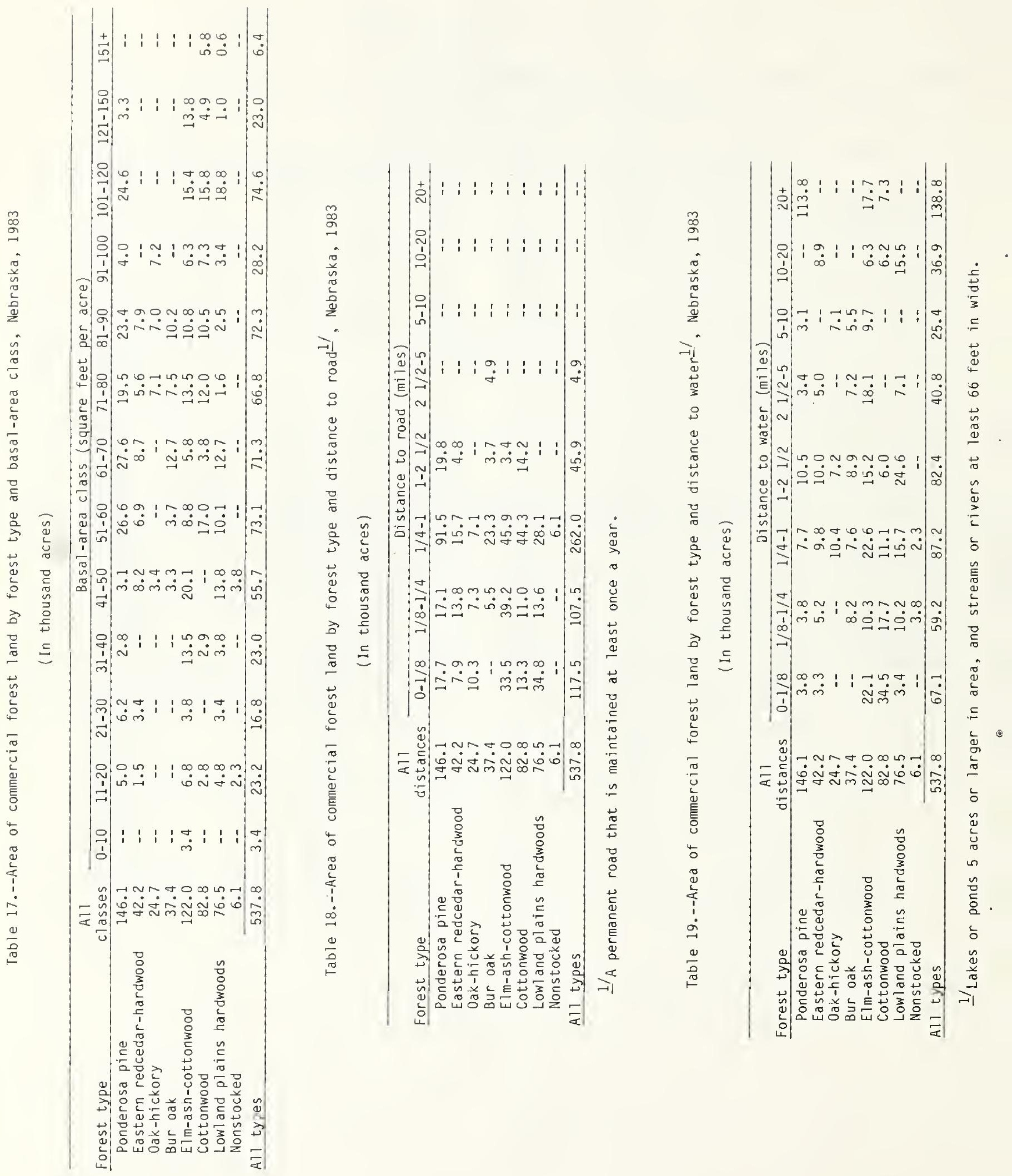



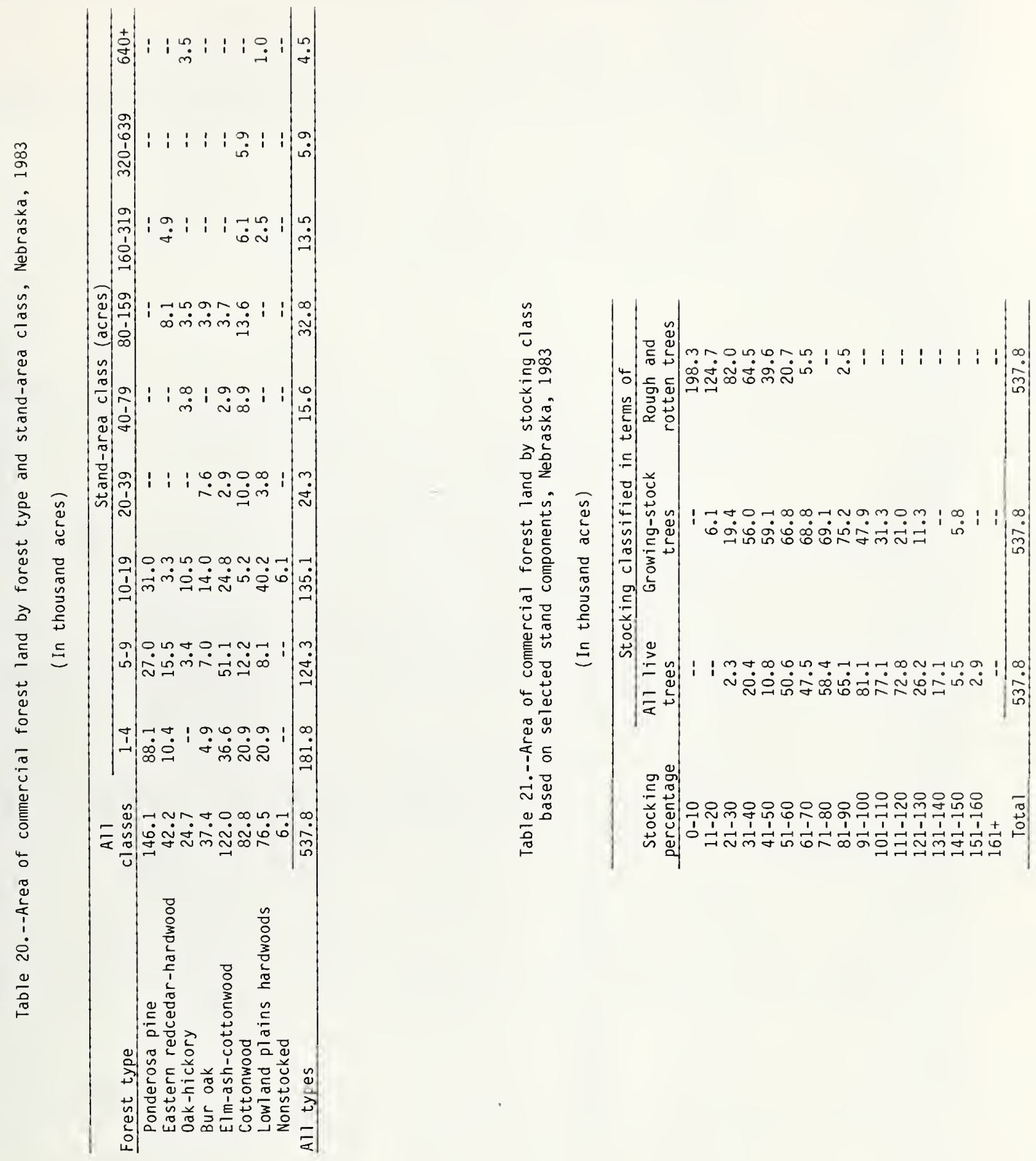
Table 22.--Area of commercial forest land by forest type, physiographic class, and ownership class, Nebraska, 1983

(In thousand acres)

\begin{tabular}{|c|c|c|c|c|c|c|c|c|}
\hline \multirow[b]{2}{*}{$\begin{array}{l}\text { Forest type and } \\
\text { physiographic class }\end{array}$} & \multirow[b]{2}{*}{$\begin{array}{c}\text { All } \\
\text { classes }\end{array}$} & \multicolumn{7}{|c|}{ Ownership class } \\
\hline & & $\begin{array}{c}\text { National } \\
\text { Forest } \\
\end{array}$ & State & $\begin{array}{l}\text { County and } \\
\text { municipal }\end{array}$ & Indian & Farmer & $\begin{array}{c}\text { Misc. } \\
\text { private } \\
\text { coporation }\end{array}$ & $\begin{array}{l}\text { Misc. } \\
\text { private } \\
\text { individual }\end{array}$ \\
\hline \multicolumn{9}{|l|}{ Ponderosa pine } \\
\hline Xeric & 14.7 & -- & -- & -- & -- & 14.7 & - & -- \\
\hline Xeromesic & 111.5 & 30.8 & -- & -- & -- & 65.8 & -- & 14.9 \\
\hline Mesic & 19.9 & -- & 7.7 & -- & -- & 9.1 & -- & 3.1 \\
\hline Hydromesic & -- & -- & -- & -- & -- & -- & -- & -- \\
\hline Hydric & $\because$ & -- & -- & $\because-$ & $\therefore$ & $=$ & $\therefore$ & -- \\
\hline All classes & 146.1 & 30.8 & 7.7 & -- & -- & 89.6 & -- & 18.0 \\
\hline \multicolumn{9}{|c|}{ Eastern redcedar-hardwood } \\
\hline Xeric & 8.2 & -- & -- & -- & -- & 8.2 & -- & -- \\
\hline Xeromesic & 10.7 & -- & -- & -- & -- & 6.7 & 1.5 & 2.5 \\
\hline Mesic & 12.3 & -- & -- & -- & -- & 12.3 & -- & -- \\
\hline Hydromesic & 11.0 & -- & 4.9 & -- & -- & -- & -- & 6.1 \\
\hline Hydric & -- & -- & -- & -- & - & -- & -- & -- \\
\hline All classes & 42.2 & - & 4.9 & -- & -- & 27.2 & 1.5 & 8.6 \\
\hline \multicolumn{9}{|l|}{ 0ak-hickory } \\
\hline Xeric & -- & -- & -- & -- & -- & -- & -- & -- \\
\hline Xeromesic & -- & -- & -- & -- & -- & -- & -- & -- \\
\hline Mesic & 24.7 & -- & -- & -- & 3.5 & 3.7 & 3.4 & 14.1 \\
\hline Hydromesic & -- & -- & -- & -- & -- & -- & -- & -- \\
\hline Hydric & -- & -- & - & -- & $=$ & -- & -- & -- \\
\hline All classes & 24.7 & $\therefore$ & - & - & 3.5 & 3.7 & 3.4 & 14.1 \\
\hline \multicolumn{9}{|l|}{ Bur oak } \\
\hline Xeric & 4.9 & -- & -- & -- & -- & 4.9 & -- & -- \\
\hline Xeromesic & 10.1 & -- & -- & -- & -- & 7.2 & -- & 2.9 \\
\hline Mesic & 18.5 & -- & -- & -- & -- & 9.6 & 3.7 & 5.2 \\
\hline Hyd romesic & 3.9 & -- & -- & -- & -- & 3.9 & -- & -- \\
\hline Hydric & -- & $\therefore$ & $\because-$ & -- & $=$ & $\ldots$ & -- & -- \\
\hline All classes & 37.4 & $=$ & $=$ & $=$ & -- & 25.6 & 3.7 & 8.1 \\
\hline \multicolumn{9}{|l|}{ Elm-ash-cottonwood } \\
\hline Xeric & -- & -- & -- & -- & -- & -- & -- & -- \\
\hline Xeromesic & 3.4 & -- & -- & -- & -- & 3.4 & -- & -- \\
\hline Mesic & 60.3 & -- & 3.4 & -- & -- & 56.9 & -- & -- \\
\hline Hydromesic & 54.9 & -- & -- & 3.8 & -- & 41.2 & -- & 9.9 \\
\hline Hydric & 3.4 & $\because$ & $\because$ & -- & $\because-$ & 3.4 & -- & $\because$ \\
\hline All classes & 122.0 & $=$ & 3.4 & 3.8 & -- & 104.9 & $=$ & 9.9 \\
\hline \multicolumn{9}{|l|}{ Cottonwood } \\
\hline Xeric & 3.8 & -- & -- & -- & -- & 3.8 & -- & -- \\
\hline Xeromesic & -- & -- & -- & -- & -- & -- & -- & -- \\
\hline Mesic & 32.4 & -- & 2.9 & -- & -- & 25.7 & -- & 3.8 \\
\hline Hydromesic & 43.2 & -- & 2.9 & -- & 2.8 & 35.1 & -- & 2.4 \\
\hline Hydric & 3.4 & - & -- & -- & -- & -- & 3.4 & -- \\
\hline All classes & 82.8 & -- & 5.8 & -- & 2.8 & 64.6 & 3.4 & 6.2 \\
\hline \multicolumn{9}{|c|}{ Lowland plains hardwoods } \\
\hline Xeric & -- & -- & -- & -- & -- & -- & -- & -- \\
\hline Xeromesic & 8.3 & -- & -- & -- & -- & 3.4 & -- & 4.9 \\
\hline Mesic & 60.0 & -- & -- & -- & 2.5 & 51.5 & -- & 6.0 \\
\hline Hydromesic & 8.2 & -- & -- & -- & -- & 8.2 & -- & -- \\
\hline Hydric & -- & $\therefore$ & $\because$ & - & $=$ & -- & -- & -- \\
\hline All classes & 76.5 & -- & -- & -- & 2.5 & 63.1 & -- & 10.9 \\
\hline \multicolumn{9}{|l|}{ Nonstocked } \\
\hline Xeric & 2.3 & -- & -- & -- & -- & 2.3 & -- & -- \\
\hline Xeromesic & -- & -- & -- & -- & -- & -- & -- & -- \\
\hline Mesic & -- & -- & -- & -- & -- & -- & -- & -- \\
\hline Hydromesic & 3.8 & -- & -- & -- & -- & -- & -- & 3.8 \\
\hline Hydric & $\because$ & $=$ & $=$ & -- & $=$ & -- & - & -- \\
\hline All classes & 6.1 & -- & -- & $=-$ & $=$ & 2.3 & $=$ & 3.8 \\
\hline \multicolumn{9}{|l|}{ All types } \\
\hline Xeric & 33.9 & -- & -- & -- & -- & 33.9 & -- & -- \\
\hline Xeromesic & 144.0 & 30.8 & -- & -- & -- & 86.5 & 1.5 & 25.2 \\
\hline Mesic & 228.1 & -- & 14.0 & -- & 6.0 & 168.8 & 7.1 & 32.2 \\
\hline Hydromesic & 125.0 & -- & 7.8 & 3.8 & 2.8 & 88.4 & -- & 22.2 \\
\hline Hydric & 6.8 & $=$ & $=$ & -- & -- & 3.4 & 3.4 & -- \\
\hline Al1 classes & 537.8 & 30.8 & 21.8 & 3.8 & 8.8 & 381.0 & 12.0 & 79.6 \\
\hline
\end{tabular}


Table 23.--Area of noncommercial forest land by ownership class, Nebraska, 1983

(In thousand acres)

\begin{tabular}{lrrr}
\hline Ownership class & Total & Unproductive & $\begin{array}{c}\text { Productive- } \\
\text { reserved }\end{array}$ \\
\hline National Forest & 3.1 & 3.1 & -- \\
Miscellaneous federal & 5.1 & 3.8 & 1.3 \\
State & 28.3 & 11.8 & 16.5 \\
County and municipal & 0.4 & -.5 & 0.4 \\
Indian & 3.5 & 3.5 & -- \\
Farmer & 104.7 & 104.7 & -- \\
Misc. private corporation & 8.4 & 8.4 & $\ldots$ \\
Misc. private individual & 27.0 & 27.0 & 18.2 \\
All owners & 180.5 & 162.3 & $-\ldots$ \\
\hline
\end{tabular}

Table 24.--Area of noncommercial forest land by forest type and Forest Survey Unit, Nebraska, 1983

(In thousand acres)

ALL UNITS

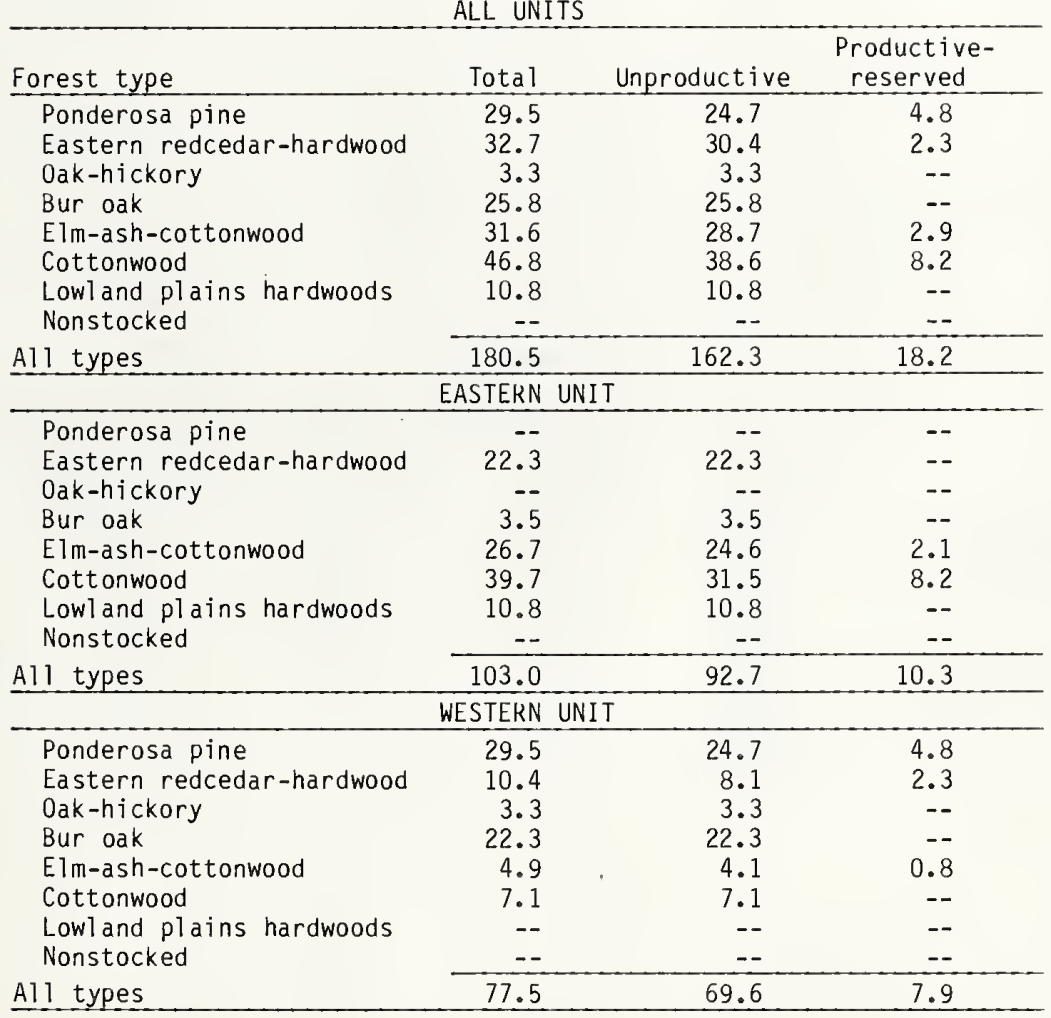


Table 25.--Area of nonforest land with trees by land use, forest type, and Forest Survey Unit, Nebraska, 1983

(In thousand acres)

ALL UNITS

\begin{tabular}{|c|c|c|c|c|c|c|c|c|c|}
\hline \multirow[b]{2}{*}{ Land use } & \multirow[b]{2}{*}{$\begin{array}{l}\text { All } \\
\text { types }\end{array}$} & \multicolumn{8}{|c|}{ Forest type } \\
\hline & & $\begin{array}{l}\text { Ponderosa } \\
\text { pine }\end{array}$ & $\begin{array}{l}\text { Eastern } \\
\text { redcedar- } \\
\text { hardwood }\end{array}$ & $\begin{array}{c}\text { Oak- } \\
\text { hickory }\end{array}$ & $\begin{array}{l}\text { Bur } \\
\text { oak }\end{array}$ & $\begin{array}{c}\text { Elm-ash- } \\
\text { cottonwood }\end{array}$ & Cottonwood & $\begin{array}{l}\text { Lowland } \\
\text { plains } \\
\text { hardwoods }\end{array}$ & Nonstocked \\
\hline Cropland & 41.8 & 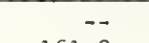 & -- & 3.5 & 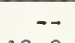 & 34.9 & 3.4 & -- & -- \\
\hline Improved pasture & 582.5 & 161.3 & 79.4 & 6.8 & 13.9 & 215.0 & 78.7 & 10.2 & 17.2 \\
\hline Wooded strips & 262.2 & 25.3 & 13.2 & 10.2 & 20.5 & 113.9 & 68.9 & 10.2 & -- \\
\hline Marsh & 7.8 & -- & - & -- & -- & 7.8 & -- & -- & -- \\
\hline Windbreaks & 139.1 & -- & 21.3 & 6.9 & -- & 82.1 & 25.3 & 3.5 & -- \\
\hline Wooded pasture & 78.3 & 6.2 & -- & 3.8 & 16.0 & 10.5 & 18.9 & 5.3 & 17.6 \\
\hline All uses & $1,111.7$ & 192.8 & 113.9 & 31.2 & 50.4 & 464.2 & 195.2 & 29.2 & 34.8 \\
\hline \multicolumn{10}{|c|}{ EASTERN UNIT } \\
\hline Cropland & 31.5 & -- & -- & -- & - & 31.5 & -- & -- & -- \\
\hline Improved pasture & 221.6 & -- & 38.7 & 3.4 & -- & 135.2 & 34.1 & 10.2 & -- \\
\hline Wooded strips & 159.0 & -- & 9.8 & 3.4 & 6.8 & 89.2 & 43.0 & 6.8 & -- \\
\hline Marsh & 2.3 & -- & -- & -- & -- & 2.3 & -- & $\rightarrow$ & -- \\
\hline Windbreaks & 90.8 & -- & 12.8 & 6.9 & -- & 57.6 & 13.5 & -- & -- \\
\hline Wooded pasture & 35.2 & $\therefore$ & -- & 3.8 & 9.3 & 10.5 & 2.9 & 2.9 & 5.8 \\
\hline All uses & 540.4 & $\cdots$ & 61.3 & 17.5 & 16.1 & 326.3 & 93.5 & 19.9 & 5.8 \\
\hline \multicolumn{10}{|c|}{ WESTERN UNIT } \\
\hline Cropl and & 10.3 & -- & -- & 3.5 & -- & 3.4 & 3.4 & -- & -- \\
\hline Improved pasture & 360.9 & 161.3 & 40.7 & 3.4 & 13.9 & 79.8 & 44.6 & -- & 17.2 \\
\hline Wooded strips & 103.2 & 25.3 & 3.4 & 6.8 & 13.7 & 24.7 & 25.9 & 3.4 & -- \\
\hline Marsh & 5.5 & -- & -- & -- & -- & 5.5 & -. & -- & -- \\
\hline Windbreaks & 48.3 & -- & 8.5 & -- & -- & 24.5 & 11.8 & 3.5 & -- \\
\hline Wooded pasture & 43.1 & 6.2 & -- & -- & 6.7 & -- & 16.0 & 2.4 & 11.8 \\
\hline All uses & 571.3 & 192.8 & 52.6 & 13.7 & 34.3 & 137.9 & 101.7 & 9.3 & 29.0 \\
\hline
\end{tabular}

Table 26.--Area of nonforest land with trees by forest type and stand-size class, Nebraska, 1983

(In thousand acres)

\begin{tabular}{|c|c|c|c|c|c|}
\hline \multirow[b]{2}{*}{ Forest type } & \multirow[b]{2}{*}{$\begin{array}{l}\text { All } \\
\text { stands }\end{array}$} & \multicolumn{4}{|c|}{ Stand-size class } \\
\hline & & $\begin{array}{c}\text { Sawt imber } \\
\text { stands }\end{array}$ & $\begin{array}{c}\text { Poletimber } \\
\text { stands }\end{array}$ & $\begin{array}{l}\text { Sapling and } \\
\text { seedling stands }\end{array}$ & $\begin{array}{c}\text { Nonstocked } \\
\text { areas }\end{array}$ \\
\hline $\begin{array}{l}\text { Ponderosa pine } \\
\text { Eastern redcedar-hardwood } \\
\text { 0ak-hickory } \\
\text { Bur oak } \\
\text { Elm-ash-cottonwood } \\
\text { Cottonwood } \\
\text { Lowland plains hardwoods } \\
\text { Nonstocked }\end{array}$ & $\begin{array}{r}192.8 \\
113.9 \\
31.2 \\
50.4 \\
464.2 \\
195.2 \\
29.2 \\
34.8\end{array}$ & $\begin{array}{r}93.4 \\
6.6 \\
6.9 \\
20.2 \\
149.6 \\
121.7 \\
13.6 \\
--\end{array}$ & $\begin{array}{r}72.8 \\
45.7 \\
13.7 \\
24.2 \\
240.8 \\
63.3 \\
10.3 \\
--\end{array}$ & $\begin{array}{r}26.6 \\
61.6 \\
10.6 \\
6.0 \\
73.8 \\
10.2 \\
5.3 \\
--\end{array}$ & $\begin{array}{l}-- \\
-- \\
-- \\
-- \\
-- \\
-- \\
-- \\
34.8\end{array}$ \\
\hline All types & $1,111.7$ & 412.0 & 470.8 & 194.1 & 34.8 \\
\hline
\end{tabular}


Table 27.--Area of windbreaks by forest type, stand-size class, and Forest Survey Unit, Nebraska, 1983

(In thousand acres)

ALL UNITS

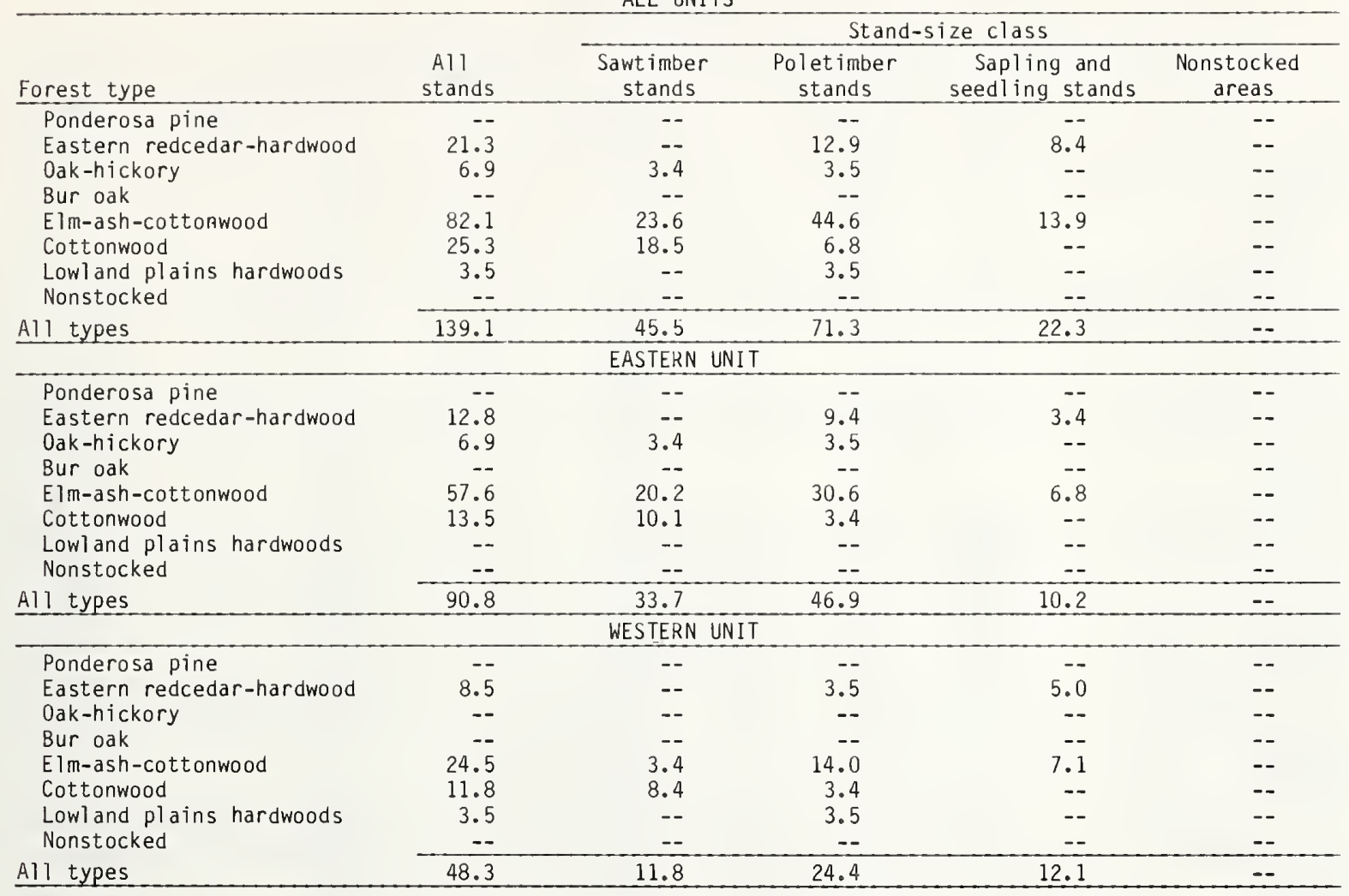


Table 28.--Area of wooded strips by forest type, stand-size class, and ownership class, Nebraska, 1983

(In thousand acres)

\begin{tabular}{|c|c|c|c|c|c|c|c|c|}
\hline \multirow[b]{2}{*}{$\begin{array}{l}\text { Forest type and } \\
\text { stand-size class }\end{array}$} & \multirow[b]{2}{*}{$\begin{array}{c}\text { All } \\
\text { classes }\end{array}$} & \multicolumn{7}{|c|}{ Ownership class } \\
\hline & & $\begin{array}{c}\text { National } \\
\text { Forest }\end{array}$ & State & $\begin{array}{l}\text { County and } \\
\text { municipal }\end{array}$ & Indian & Farmer & $\begin{array}{l}\text { Misc. } \\
\text { private } \\
\text { coporation }\end{array}$ & $\begin{array}{l}\text { Misc. } \\
\text { private } \\
\text { individual }\end{array}$ \\
\hline \multicolumn{9}{|l|}{ Ponderosa pine } \\
\hline Sawtimber & 15.3 & -- & -- & -- & -- & 15.3 & -- & -- \\
\hline Poletimber & 10.0 & 3.6 & -- & -- & -- & 6.4 & -- & -- \\
\hline Sapling \& seedling & $\ldots$ & $\therefore-$ & $=-$ & -- & $=-$ & $\therefore$ & -- & -- \\
\hline All stands & 25.3 & 3.6 & - & -- & $\cdots$ & 21.7 & -- & $\because$ \\
\hline \multicolumn{9}{|c|}{ Eastern redcedar-hardwood } \\
\hline Sawt imber & -- & -- & -- & -- & -- & -- & -- & -- \\
\hline Poletimber & 13.2 & -- & -- & -- & -- & 13.2 & -- & -- \\
\hline Sapling \& seedling & $=$ & - & - & $\because$ & - & -- & $\because$ & -- \\
\hline All stands & 13.2 & $=$ & $=$ & $=$ & $\because$ & 13.2 & - & -- \\
\hline \multicolumn{9}{|l|}{ 0ak-hickory } \\
\hline Sawt imber & -- & -- & -- & -- & -- & -- & -- & - \\
\hline Poletimber & 6.8 & -- & -- & -- & -- & 6.8 & -- & -- \\
\hline Sapling \& seedling & 3.4 & -- & -- & $=$ & -- & -- & -- & 3.4 \\
\hline All stands & 10.2 & -- & -- & -- & -- & 6.8 & -- & 3.4 \\
\hline \multicolumn{9}{|l|}{ Bur oak } \\
\hline Sawt imber & 6.8 & -- & 3.4 & -- & -- & 3.4 & -- & -- \\
\hline Polet imber & 13.7 & -- & -- & -- & -- & 13.7 & -- & -- \\
\hline Sapling \& seedling & $=-$ & -- & -- & -- & -- & -- & -- & -- \\
\hline All stands & 20.5 & -- & 3.4 & -- & -- & 17.1 & -- & -- \\
\hline \multicolumn{9}{|l|}{ Elm-ash-cottonwood } \\
\hline Sawt imber & 34.4 & -- & -- & -- & -- & 34.4 & -- & -- \\
\hline Poletimber & 69.2 & -- & -- & -- & -- & 69.2 & -- & -- \\
\hline Sapling \& seedling & 10.3 & -- & -- & -- & -- & 10.3 & -- & -- \\
\hline All stands & 113.9 & $=-$ & $=$ & $=$ & $\because-$ & 113.9 & -- & -- \\
\hline \multicolumn{9}{|l|}{ Cottonwood } \\
\hline Sawtimber & 33.2 & -- & -- & -- & -- & 30.3 & -- & 2.9 \\
\hline Poletimber & 32.3 & -- & -- & -- & -- & 28.8 & -- & 3.5 \\
\hline Sapling \& seedling & 3.4 & -- & -- & $=$ & -- & 3.4 & -- & $\therefore$ \\
\hline Al1 stands & 68.9 & $\because$ & -- & -- & -- & 62.5 & -- & 6.4 \\
\hline \multicolumn{9}{|c|}{ Lowl and plains hardwoods } \\
\hline Sawtimber & 3.4 & -- & -- & -- & -- & 3.4 & -- & -- \\
\hline Poletimber & 6.8 & -- & -- & -- & -- & 3.4 & -- & 3.4 \\
\hline Sapling \& seedling & -- & -- & - & -- & -- & - & -- & -- \\
\hline All stands & 10.2 & -- & -- & -- & $=$ & 6.8 & -- & 3.4 \\
\hline Nonst ocked & -- & -- & -- & $=$ & -- & - & $=-$ & $\because$ \\
\hline \multicolumn{9}{|l|}{ A11 types } \\
\hline Sawt imber & 93.1 & -- & 3.4 & -- & -- & 86.8 & -- & 2.9 \\
\hline Poletimber & 152.0 & 3.6 & -- & -- & -- & 141.5 & -- & 6.9 \\
\hline Sapling \& seedling & 17.1 & -- & -- & -- & -- & 13.7 & -- & 3.4 \\
\hline Nonstocked & -- & -- & - & -- & -- & $=-$ & -- & - \\
\hline Al1 stands & 262.2 & 3.6 & 3.4 & -- & $\therefore$ & 242.0 & - & 13.2 \\
\hline
\end{tabular}



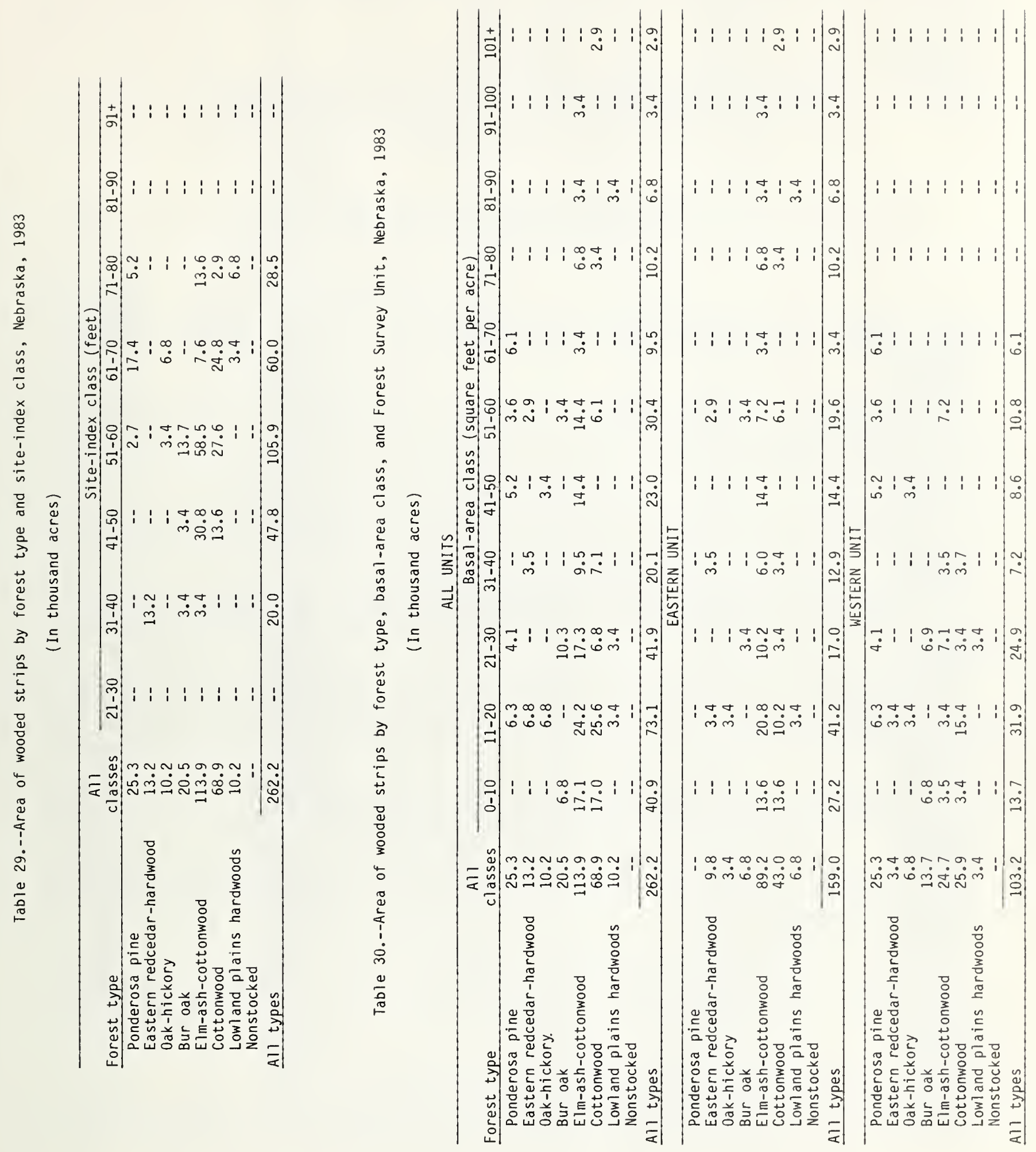
Table 31.--Area of wooded strips by forest type and stand-age class, Nebraska, 1983

(In thousand acres)

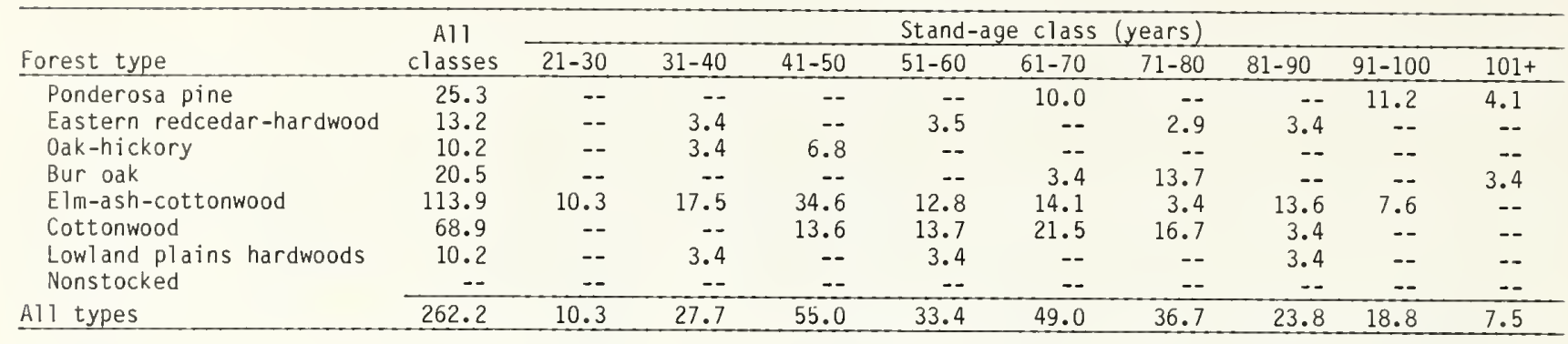

Table 32.--Area of wooded strips by forest type, stand-size class, and ownership class, Nebraska, 1983

(In thousand acres)

\begin{tabular}{|c|c|c|c|c|c|c|c|c|}
\hline \multirow[b]{2}{*}{$\begin{array}{l}\text { Forest type and } \\
\text { stand-size class }\end{array}$} & \multirow[b]{2}{*}{$\begin{array}{c}\text { All } \\
\text { classes }\end{array}$} & \multicolumn{7}{|c|}{ Ownership class } \\
\hline & & $\begin{array}{l}\text { National } \\
\text { Forest }\end{array}$ & State & $\begin{array}{l}\text { County and } \\
\text { municipal }\end{array}$ & Indian & Farmer & $\begin{array}{l}\text { Misc. } \\
\text { private } \\
\text { coporation }\end{array}$ & $\begin{array}{l}\text { Misc. } \\
\text { private } \\
\text { individual }\end{array}$ \\
\hline \multicolumn{9}{|l|}{ Ponderosa pine } \\
\hline Sawt imber & 15.3 & -- & -- & -- & -- & 15.3 & -- & -- \\
\hline Poletimber & 10.0 & 3.6 & -- & -- & -- & 6.4 & -- & -- \\
\hline Sapling \& seedling & -- & -- & -- & -- & -- & - & -- & -- \\
\hline Al1 stands & 25.3 & 3.6 & $\therefore-$ & $\therefore$ & $\because$ & 21.7 & -- & -- \\
\hline \multicolumn{9}{|c|}{ Eastern redcedar-hardwood } \\
\hline Sawtimber & -- & -- & -- & -- & -- & -- & -- & -- \\
\hline Poletimber & 13.2 & -- & -- & -. & -- & 13.2 & -- & -- \\
\hline Sapling \& seedling & -- & -- & -- & $\cdots$ & -- & -- & -- & -- \\
\hline A11 stands & 13.2 & $\because-$ & $\therefore$ & -- & -- & 13.2 & -- & -- \\
\hline \multicolumn{9}{|l|}{ oak-hickory } \\
\hline Sawtimber & -- & - & -- & - & - & -- & -- & -- \\
\hline Poletimber & 6.8 & -- & -- & -- & -- & 6.8 & -- & -- \\
\hline Sapling \& seedling & 3.4 & -- & -- & -- & -- & -- & - & 3.4 \\
\hline A11 stands & 10.2 & -- & $=-$ & $\therefore$ & $\therefore$ & 6.8 & $\therefore$ & 3.4 \\
\hline \multicolumn{9}{|l|}{ Bur oak } \\
\hline Sawt imber & 6.8 & -- & 3.4 & -- & -- & 3.4 & -- & -- \\
\hline Poletimber & 13.7 & -- & -- & -- & -- & 13.7 & -- & -- \\
\hline Sapling \& seedling & $=-$ & -- & -- & -- & -- & -- & -- & -- \\
\hline A11 stands & 20.5 & - & 3.4 & $-=$ & - & 17.1 & -- & -- \\
\hline \multirow{2}{*}{\multicolumn{9}{|c|}{ Elm-ash-cottonwood }} \\
\hline & 34.4 & -- & -- & -- & -- & 34.4 & -- & -. \\
\hline Poletimber & 69.2 & -- & -- & -- & -- & 69.2 & -- & -- \\
\hline Sapling \& seedling & 10.3 & -- & -- & $=$ & - & 10.3 & -- & -- \\
\hline All stands & 113.9 & -- & -- & -- & $\cdots$ & 113.9 & -- & -- \\
\hline \multicolumn{9}{|l|}{ Cottonwood } \\
\hline Sawt imber & 33.2 & -- & -- & -- & -- & 30.3 & -- & 2.9 \\
\hline Poletimber & 32.3 & -- & -- & -- & -- & 28.8 & -- & 3.5 \\
\hline Sapling \& seedling & 3.4 & -- & -- & -- & -- & 3.4 & -- & $\ldots$ \\
\hline All stands & 68.9 & -- & $\cdots$ & -- & -- & 62.5 & -- & 6.4 \\
\hline \multicolumn{9}{|c|}{ Lowland plains hardwoods } \\
\hline Sawt imber & 3.4 & -- & -- & -- & -- & 3.4 & -- & -- \\
\hline Poletimber & 6.8 & -- & -- & -- & -- & 3.4 & -- & 3.4 \\
\hline Sapling \& seedling &.- & $\cdots$ & $\therefore$ & -- & - & $\because$ & -- & -- \\
\hline A11 stands & 10.2 & $=$ & -- & $=$ & -- & 6.8 & $=$ & 3.4 \\
\hline Nonstocked & $=-$ & -- & $=$ & -- & $=$ & -- & $\because$ & -- \\
\hline \multicolumn{9}{|l|}{ All types } \\
\hline Sawt imber & 93.1 & -- & 3.4 & -- & -- & 86.8 & -- & 2.9 \\
\hline Poletimber & 152.0 & 3.6 & -- & -- & -- & 141.5 & -- & 6.9 \\
\hline Sapling \& seedling & 17.1 & -- & -- & -- & -- & 13.7 & -- & 3.4 \\
\hline Nonstocked & -- & -- & - & -- & -- & -- & $=-$ & -- \\
\hline All stands & 262.2 & 3.6 & 3.4 & $\cdots$ & -- & 242.0 & - & 13.2 \\
\hline
\end{tabular}


Table 33.--Area of wooded strips in private ownership by ownership class, owner tenure, and size of holding, Nebraska, 1983

(In thousand acres)

ALL UNITS

\begin{tabular}{|c|c|c|c|c|c|c|}
\hline \multirow{2}{*}{$\begin{array}{l}\text { Ownership class } \\
\text { and owner tenure }\end{array}$} & \multirow{2}{*}{$\begin{array}{c}\text { All } \\
\text { sizes }\end{array}$} & \multicolumn{5}{|c|}{ Size of holding (acres) } \\
\hline & & $1-5$ & $5-10$ & $10-20$ & $20-50$ & $50+$ \\
\hline $\begin{array}{l}\text { Farmer } \\
1-4 \text { years } \\
5-9 \text { years } \\
10-19 \text { years } \\
20+\text { years }\end{array}$ & $\begin{array}{r}214.2 \\
-- \\
3.5 \\
24.3 \\
\end{array}$ & $\begin{array}{r}63.9 \\
-- \\
-- \\
7.2 \\
\end{array}$ & $\begin{array}{r}79.0 \\
-- \\
-- \\
5.3\end{array}$ & $\begin{array}{r}54.3 \\
\cdots \\
\cdots \\
-- \\
\end{array}$ & $\begin{array}{r}17.0 \\
-- \\
3.5 \\
11.8 \\
\end{array}$ & $\begin{array}{l}-- \\
-- \\
-- \\
--\end{array}$ \\
\hline & 242.0 & 71.1 & 84.3 & 54.3 & 32.3 & -- \\
\hline $\begin{array}{c}\text { Miscellaneous pri } \\
\text { corporation } \\
1-4 \text { years } \\
5-9 \text { years } \\
10-19 \text { years } \\
20+\text { years }\end{array}$ & $\begin{array}{l}-- \\
-- \\
-- \\
--\end{array}$ & $\begin{array}{l}-- \\
-- \\
-\end{array}$ & $\begin{array}{l}-- \\
-- \\
-- \\
--\end{array}$ & $\begin{array}{l}-- \\
-- \\
-- \\
-- \\
\end{array}$ & $\begin{array}{l}-- \\
-- \\
-- \\
--\end{array}$ & $\begin{array}{l}-- \\
-- \\
--\end{array}$ \\
\hline Total & $=$ & $=$ & $=$ & $\because$ & -- & $\therefore-$ \\
\hline $\begin{array}{l}\text { Miscellaneous pri } \\
\text { individual } \\
1-4 \text { years } \\
5-9 \text { years } \\
10-19 \text { years } \\
20+\text { years }\end{array}$ & $\begin{array}{r}9.8 \\
-- \\
3.4 \\
-- \\
\end{array}$ & $\begin{array}{l}-- \\
-- \\
-- \\
--\end{array}$ & $\begin{array}{c}6.4 \\
-- \\
-- \\
-- \\
\end{array}$ & $\begin{array}{r}3.4 \\
-- \\
3.4 \\
-2 \\
\end{array}$ & $\begin{array}{l}-- \\
-- \\
-- \\
-- \\
\end{array}$ & $\begin{array}{l}-- \\
-- \\
-- \\
--\end{array}$ \\
\hline Total & 13.2 & $=-$ & 6.4 & 6.8 & -- & - \\
\hline $\begin{array}{l}\text { A11 owners } \\
1-4 \text { years } \\
5-9 \text { years } \\
10-19 \text { years } \\
20+\text { years }\end{array}$ & $\begin{array}{r}224.0 \\
-- \\
6.9 \\
24.3 \\
\end{array}$ & $\begin{array}{r}63.9 \\
-- \\
-- \\
7.2 \\
\end{array}$ & $\begin{array}{r}85.4 \\
-- \\
-- \\
5.3 \\
\end{array}$ & $\begin{array}{r}57.7 \\
-- \\
3.4 \\
-- \\
\end{array}$ & $\begin{array}{r}17.0 \\
-- \\
3.5 \\
11.8 \\
\end{array}$ & $\begin{array}{l}-- \\
-- \\
-- \\
--\end{array}$ \\
\hline Total & 255.2 & 71.1 & 90.7 & 61.1 & 32.3 & $=$ \\
\hline \multicolumn{7}{|c|}{ EASTERN UNIT } \\
\hline $\begin{array}{l}\text { Farmer } \\
1-4 \text { years } \\
5-9 \text { years } \\
10-19 \text { years } \\
20+\text { years }\end{array}$ & $\begin{array}{r}139.8 \\
-- \\
-- \\
9.4 \\
\end{array}$ & $\begin{array}{r}41.1 \\
-- \\
-- \\
3.4 \\
\end{array}$ & $\begin{array}{r}64.5 \\
-- \\
-- \\
2.6 \\
\end{array}$ & $\begin{array}{r}24.0 \\
-- \\
-- \\
-- \\
\end{array}$ & $\begin{array}{r}10.2 \\
-- \\
-- \\
3.4 \\
\end{array}$ & $\begin{array}{l}-- \\
-- \\
-- \\
--\end{array}$ \\
\hline Total & 149.2 & 44.5 & 67.1 & 24.0 & 13.6 & - \\
\hline $\begin{array}{c}\text { Miscellaneous pri } \\
\text { corporation } \\
1-4 \text { years } \\
5-9 \text { years } \\
10-19 \text { years } \\
20+\text { years }\end{array}$ & $\begin{array}{l}-- \\
-- \\
-- \\
-- \\
\end{array}$ & $\begin{array}{l}-- \\
-- \\
-- \\
\end{array}$ & $\begin{array}{l}-- \\
-- \\
-- \\
\end{array}$ & $\begin{array}{l}-- \\
-- \\
-- \\
-\end{array}$ & $\begin{array}{l}-- \\
-- \\
-- \\
--\end{array}$ & $\begin{array}{l}-- \\
-- \\
-- \\
--\end{array}$ \\
\hline Total & -- & $=$ & -- & $=$ & -- & $\cdots$ \\
\hline $\begin{array}{l}\text { Miscellaneous pri } \\
\text { individual } \\
1-4 \text { years } \\
5-9 \text { years } \\
10-19 \text { years } \\
20+\text { years }\end{array}$ & $\begin{array}{c}9.8 \\
-- \\
-- \\
-- \\
\end{array}$ & $\begin{array}{l}-- \\
-- \\
-- \\
\end{array}$ & $\begin{array}{r}6.4 \\
-- \\
-- \\
-- \\
\end{array}$ & $\begin{array}{c}3.4 \\
-- \\
-- \\
-- \\
\end{array}$ & $\begin{array}{l}-- \\
-- \\
-- \\
--\end{array}$ & $\begin{array}{l}-- \\
-- \\
--\end{array}$ \\
\hline Total & 9.8 & -- & 6.4 & 3.4 & -- & -- \\
\hline $\begin{array}{l}\text { A11 owners } \\
1-4 \text { years } \\
5-9 \text { years } \\
10-19 \text { years } \\
20+\text { years }\end{array}$ & $\begin{array}{r}149.6 \\
-- \\
-- \\
9.4 \\
\end{array}$ & $\begin{array}{r}41.1 \\
-- \\
-- \\
3.4 \\
\end{array}$ & $\begin{array}{r}70.9 \\
-- \\
-- \\
2.6 \\
\end{array}$ & $\begin{array}{r}27.4 \\
-- \\
-- \\
-- \\
\end{array}$ & $\begin{array}{r}10.2 \\
-- \\
-- \\
3.4 \\
\end{array}$ & $\begin{array}{l}-- \\
-- \\
-- \\
--\end{array}$ \\
\hline Total & 159.0 & 44.5 & 73.5 & 27.4 & 13.6 & $=-$ \\
\hline
\end{tabular}


(Table 33 continued)

WESTERN UNIT

\begin{tabular}{|c|c|c|c|c|c|c|}
\hline \multirow{2}{*}{$\begin{array}{l}\text { Ownership class } \\
\text { and owner tenure }\end{array}$} & \multirow{2}{*}{$\begin{array}{c}\text { All } \\
\text { sizes }\end{array}$} & \multicolumn{5}{|c|}{ Size of holding (acres) } \\
\hline & & $1-5$ & $5-10$ & $10-20$ & $20-50$ & $50+$ \\
\hline \multicolumn{7}{|l|}{ Farmer } \\
\hline $1-4$ years & 74.4 & 22.8 & 14.5 & 30.3 & 6.8 & -- \\
\hline $5-9$ years & & -- & -- & - & -- & - \\
\hline $10-19$ years & 3.5 & -- & -- & -- & 3.5 & -- \\
\hline $20+$ years & 14.9 & 3.8 & 2.7 & -- & 8.4 & -- \\
\hline Total & 92.8 & 26.6 & 17.2 & 30.3 & 18.7 & -- \\
\hline \multicolumn{7}{|c|}{ Miscellaneous private } \\
\hline \multicolumn{7}{|c|}{ corporation } \\
\hline $1-4$ years & -- & -- & -- & -- & -- & - \\
\hline $5-9$ years & -- & -- & -- & -- & -- & - \\
\hline $10-19$ years & -- & -- & -- & -- & -- & -- \\
\hline $20+$ years & -- & -- & -- & -- & -- & -- \\
\hline Total & -- & -- & -- & -- & -- & -- \\
\hline \multicolumn{7}{|c|}{$\begin{array}{l}\text { Miscellaneous private } \\
\text { individual }\end{array}$} \\
\hline $1-4$ years & -- & -- & -- & -- & -- & -- \\
\hline $5-9$ years & -- & -- & -- & -- & -- & -- \\
\hline $10-19$ years & 3.4 & -- & -- & 3.4 & -- & - \\
\hline $20+$ years & -- & -- & -- & -- & -- & -- \\
\hline Total & 3.4 & -- & -- & 3.4 & -- & -- \\
\hline \multicolumn{7}{|l|}{ All owners } \\
\hline $1-4$ years & 74.4 & 22.8 & 14.5 & 30.3 & 6.8 & -- \\
\hline $5-9$ years & -- & -- & -- & -- & -- & -- \\
\hline $10-19$ years & 6.9 & -- & -- & 3.4 & 3.5 & -- \\
\hline $20+$ years & 14.9 & 3.8 & 2.7 & -- & 8.4 & - \\
\hline Total & 96.2 & 26.6 & 17.2 & 33.7 & 18.7 & -- \\
\hline
\end{tabular}




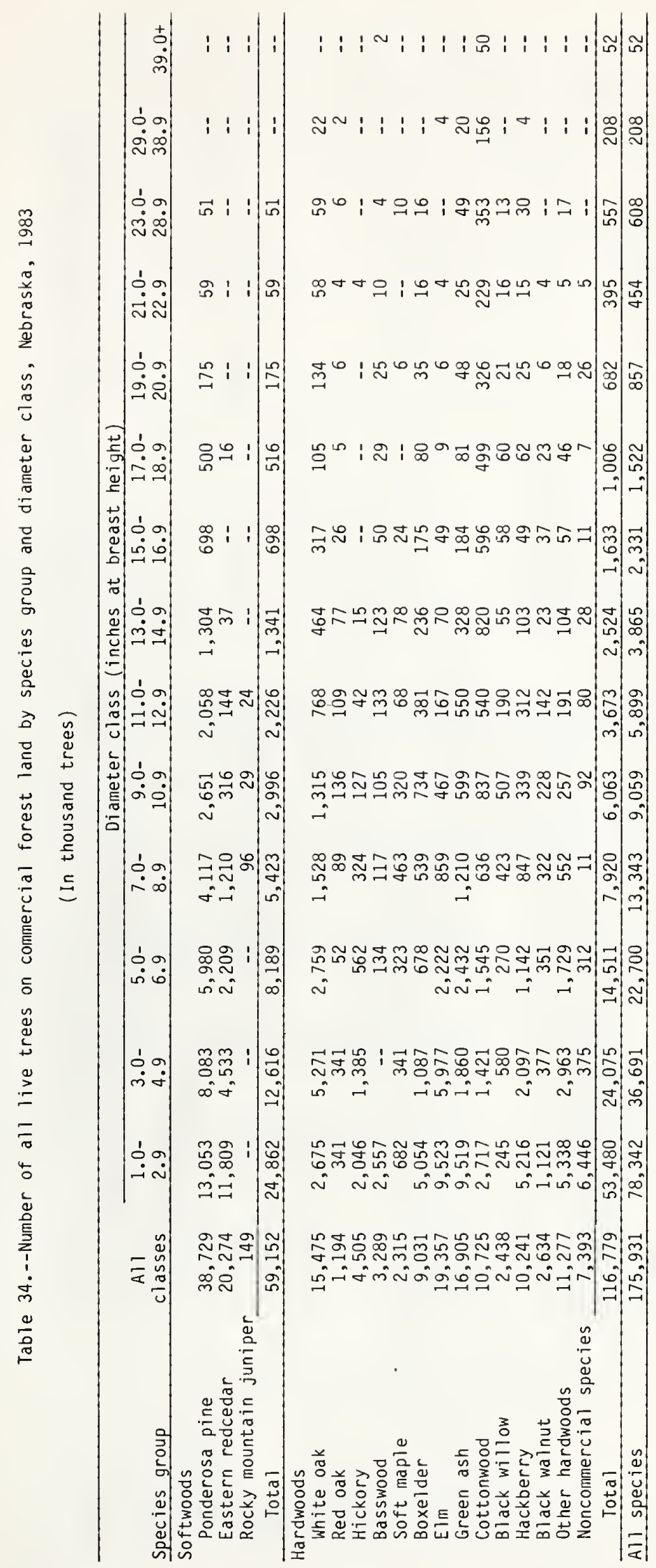




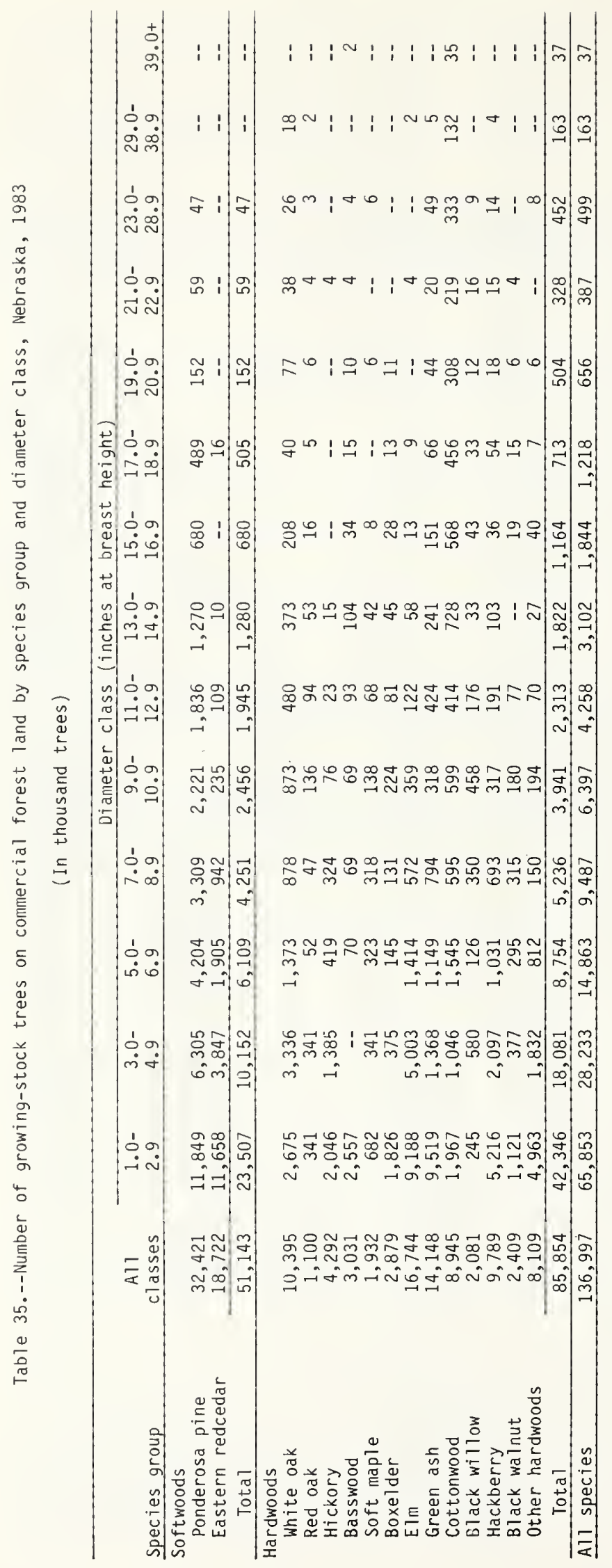


Table 36.--Number of short-log trees on commercial forest land by species group and diameter class, Nebraska, 1983

(In thousand trees)

\begin{tabular}{|c|c|c|c|c|c|c|c|c|c|c|c|}
\hline \multirow[b]{2}{*}{ Species group } & \multirow[b]{2}{*}{$\begin{array}{c}\text { All } \\
\text { classes }\end{array}$} & \multicolumn{10}{|c|}{ Diameter class (inches at breast height) } \\
\hline & & $\begin{array}{c}9.0- \\
10.9 \\
\end{array}$ & $\begin{array}{l}11.0- \\
12.9\end{array}$ & $\begin{array}{l}13.0- \\
14.9\end{array}$ & $\begin{array}{l}15.0- \\
16.9 \\
\end{array}$ & $\begin{array}{l}17.0- \\
18.9 \\
\end{array}$ & $\begin{array}{l}19.0- \\
20.9 \\
\end{array}$ & $\begin{array}{l}21.0- \\
22.9 \\
\end{array}$ & $\begin{array}{l}23.0- \\
28.9 \\
\end{array}$ & $\begin{array}{l}29.0- \\
38.9 \\
\end{array}$ & $39.0+$ \\
\hline \multicolumn{12}{|l|}{ Softwoods } \\
\hline Ponderosa pine & 367 & 171 & 148 & 24 & 18 & 6 & -- & -- & -- & -- & -- \\
\hline Eastern redcedar & 93 & 45 & 35 & 13 & -- & -- & -- & $\therefore-$ & -- & $\because$ & $=$ \\
\hline Total & 460 & 216 & 183 & 37 & 18 & 6 & -- & - & -- & $=-$ & -- \\
\hline \multicolumn{12}{|l|}{ Hardwoods } \\
\hline White oak & 309 & -- & 132 & 72 & 52 & 14 & 23 & 11 & 5 & -- & -- \\
\hline Red oak & 37 & -- & 15 & 12 & 10 & -- & -- & -- & -- & -- & -- \\
\hline Hickory & -- & -- & -- & -- & -- & -- & -- & -- & -- & -- & -- \\
\hline Basswood & 26 & -- & 24 & -- & -- & 2 & -- & -- & -- & -- & -- \\
\hline Soft maple & 48 & -- & -- & 36 & 8 & -- & -- & -- & 4 & -- & -- \\
\hline Boxelder & 94 & -- & 49 & -- & 29 & 8 & 8 & -- & -- & -- & -- \\
\hline Elm & 50 & -- & 19 & 12 & 17 & $-\rightarrow$ & -- & -- & -- & 2 & -- \\
\hline Green ash & 176 & -- & 85 & 49 & 27 & 15 & -- & -- & -- & -- & -- \\
\hline Cottonwood & 215 & -- & 63 & 68 & -- & 24 & 18 & 7 & 14 & 16 & 5 \\
\hline Black willow & 35 & -- & 14 & -- & 15 & 6 & -- & -- & -- & -- & -- \\
\hline Hackberry & 106 & -- & 74 & -- & 13 & -- & 7 & -- & 12 & -- & -- \\
\hline Black walnut & 41 & -- & 31 & -- & 10 & -- & -- & -- & -- & -- & -- \\
\hline Other hardwoods & 58 & $=-$ & 34 & 13 & $=-$ & 8 & -- & -- & 3 & -- & -- \\
\hline Total & 1,195 & $\cdots$ & 540 & 262 & 181 & 77. & 56 & 18 & 38 & 18 & 5 \\
\hline All species & 1,655 & 216 & 723 & 299 & 199 & 83 & 56 & 18 & 38 & 18 & 5 \\
\hline
\end{tabular}

Table 37.--Net volume of growing stock on commercial

forest land by species group, Nebraska, 1955 and 19831/

(In thousand cubic feet)

\begin{tabular}{lrr}
\hline & \multicolumn{2}{c}{ Growing stock } \\
\cline { 2 - 3 } Species group & 1955 & 1983 \\
\hline Softwoods & & \\
Ponderosa pine & 79,800 & 160,316 \\
Eastern redcedar & 3,800 & 9,940 \\
Jack pine & 1,000 & 170,256 \\
\cline { 2 - 3 } Total & 84,600 & \\
\hline Hardwoods & & 39,400 \\
White oak & 31,900 & 5,167 \\
Red oak & 3,400 & 4,135 \\
Hickory & 1,400 & 5,992 \\
Basswood & 5,800 & 5,381 \\
Soft maple & 1,700 & 5,294 \\
Boxelder & 1,600 & 12,413 \\
Elm & 51,300 & 25,900 \\
Green ash & 27,800 & 142,218 \\
Cottonwood & 140,100 & 11,060 \\
Black willow & 6,400 & 16,516 \\
Hackberry & 6,500 & 5,131 \\
Black walnut & 3,400 & 7,148 \\
Other hardwoods & 4,300 & 285,755 \\
$\quad$ Total & 285,600 & 456,011 \\
\hline All species & 370,200 & \\
\hline & &
\end{tabular}




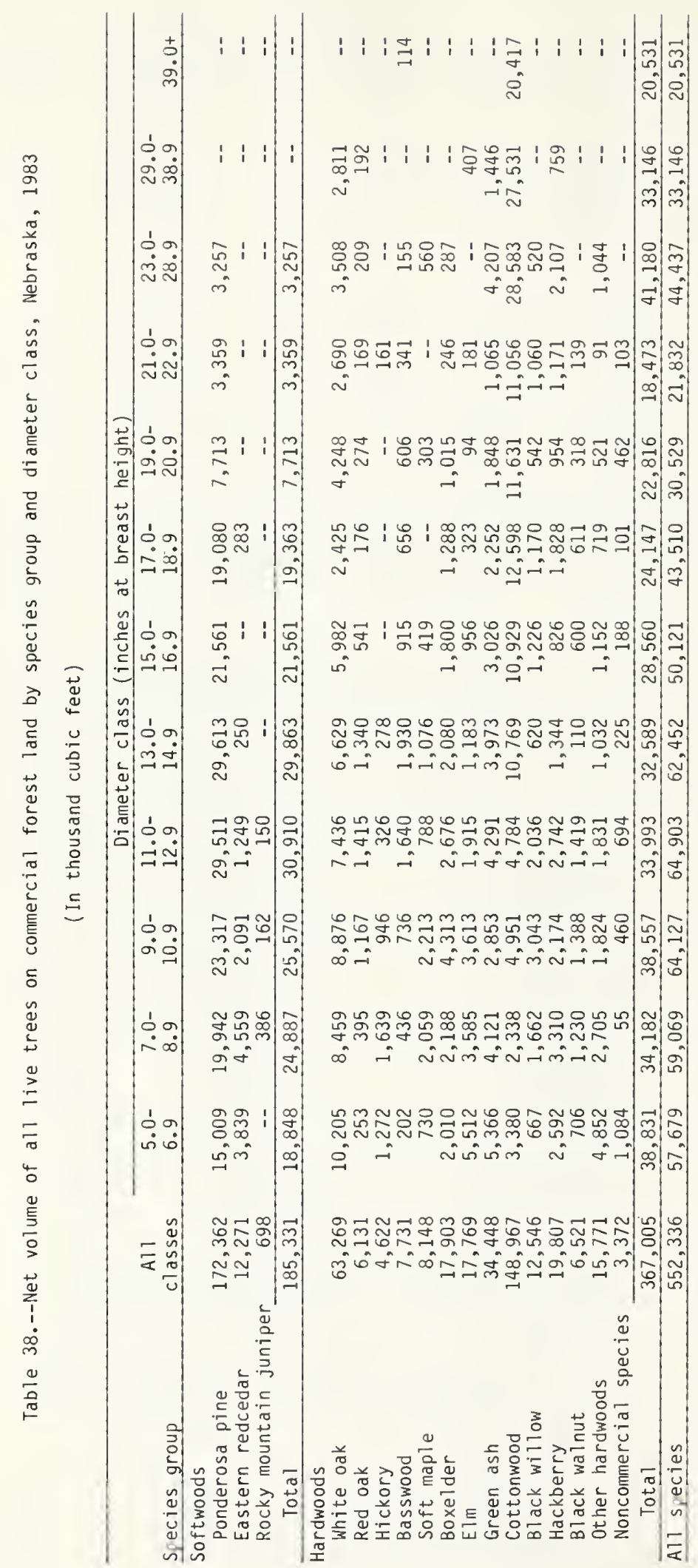


Table 39.--Net volume of timber on commercial forest land by class of timber and softwoods and hardwoods, Nebraska, 1983

(In thousand cubic feet)

\begin{tabular}{|c|c|c|c|}
\hline Class of timber & $\begin{array}{c}\text { All } \\
\text { species }\end{array}$ & Softwoods & Hardwoods \\
\hline \multicolumn{4}{|l|}{ LIVE TREES } \\
\hline \multicolumn{4}{|l|}{ Growing-stock trees } \\
\hline Saw log portion & 291,381 & 131,017 & 160,364 \\
\hline Upper stem portion & 61,667 & 5,053 & 56,614 \\
\hline Subtota 1 & 353,048 & 136,070 & 216,978 \\
\hline Poletimber & 102,963 & 34,186 & 68,777 \\
\hline Total growing stock & 456,011 & 170,256 & 285,755 \\
\hline \multicolumn{4}{|l|}{ Cull trees } \\
\hline \multicolumn{4}{|c|}{ Rough and rotten cull trees } \\
\hline Sawtimber & 24,498 & 2,841 & 21,657 \\
\hline Poletimber & 52,342 & 9,549 & 42,793 \\
\hline Subtotal & 76,840 & 12,390 & 64,450 \\
\hline Short-log trees & 19,485 & 2,685 & 16,800 \\
\hline Total cul1 & 96,325 & 15,075 & 81,250 \\
\hline TOTAL LIVE TREES & 552,336 & 185,331 & 367,005 \\
\hline SALVABLE DEAD TREES & 9,295 & 1,316 & 7,979 \\
\hline ALL CLASSES & 561,631 & 186,647 & 374,984 \\
\hline
\end{tabular}

Table 40.--Net volume of growing stock, sawtimber, short-log, and rough and rotten trees on commercial forest land by individual species, Nebraska, 1983

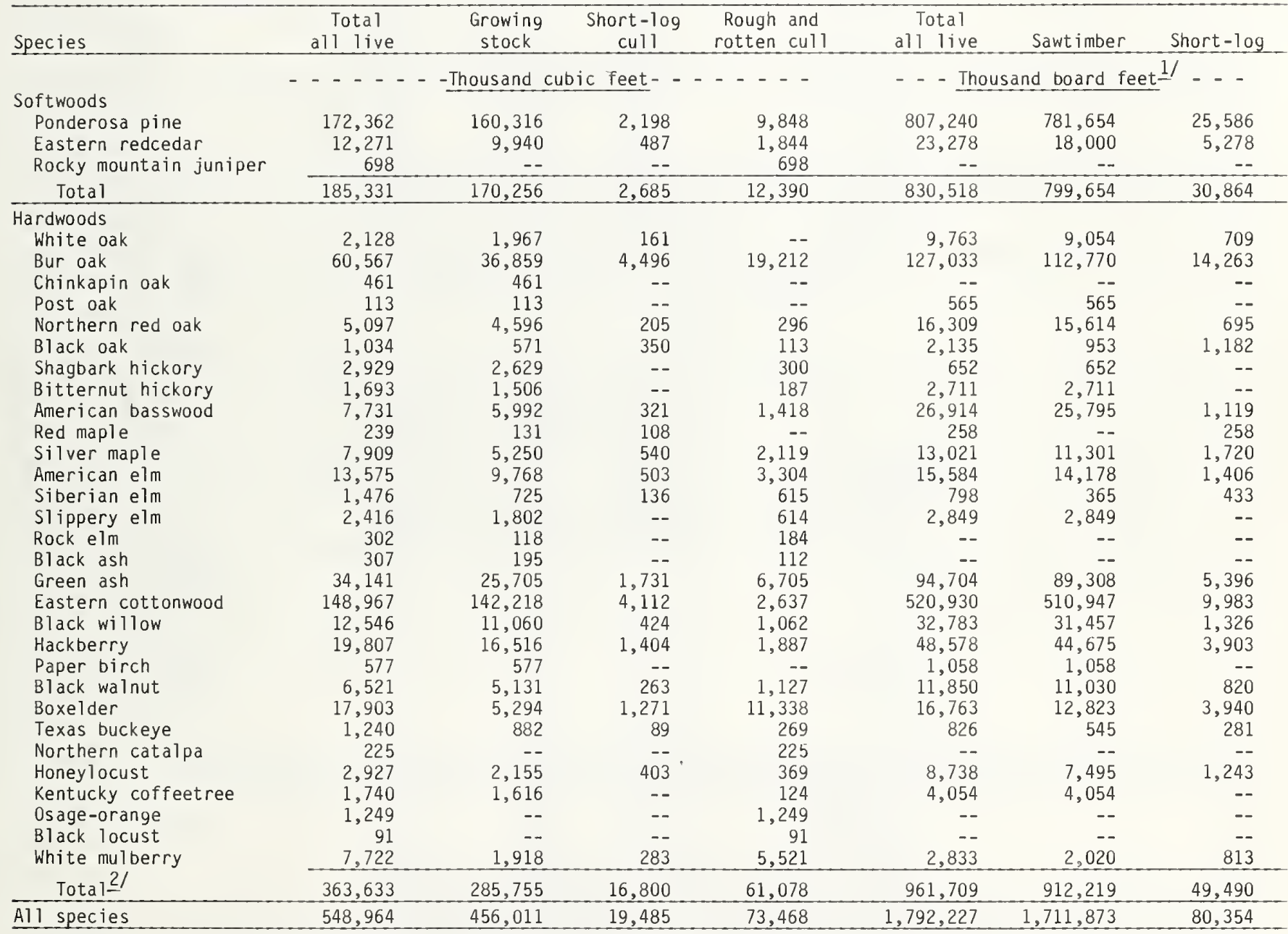

1/ International $1 / 4$-inch rule.

2I Does not include noncommercial species. See Table 41 for volumes of noncommercial species. 
Table 41.--Net volume of noncommercial species (nongrowing-stock volume) on commercial forest 1 and by individual species, Nebraska, 1983

(In thousand cubic feet)

\begin{tabular}{lc}
\hline Species & $\begin{array}{c}\text { Nongrowing-stock } \\
\text { (rough tree) volume }\end{array}$ \\
\hline Peachleaf willow & 158 \\
Diamond willow & 2,075 \\
Eastern hophornbeam & 1,139 \\
\hline All species & 3,372 \\
\hline
\end{tabular}

Table 42.--Net volume of growing stock on commercial forest land, by species group and Forest Survey Unit, Nebraska, 1983

(In thousand cubic feet)

\begin{tabular}{|c|c|c|c|}
\hline \multirow[b]{2}{*}{ Species group } & \multirow[b]{2}{*}{$\begin{array}{c}\text { All } \\
\text { Units }\end{array}$} & \multicolumn{2}{|c|}{ Forest Survey Unit } \\
\hline & & $\begin{array}{c}\text { Eastern } \\
\text { Unit }\end{array}$ & $\begin{array}{c}\text { Western } \\
\text { Unit }\end{array}$ \\
\hline $\begin{array}{l}\text { Softwoods } \\
\text { Ponderosa pine } \\
\text { Eastern redcedar }\end{array}$ & $\begin{array}{r}160,316 \\
9,940 \\
\end{array}$ & $6,17 \overline{2}$ & $\begin{array}{r}160,316 \\
3,768 \\
\end{array}$ \\
\hline Total & 170,256 & 6,172 & 164,084 \\
\hline $\begin{array}{l}\text { Hardwoods } \\
\text { White oak } \\
\text { Red oak } \\
\text { Hickory } \\
\text { Basswood } \\
\text { Soft maple } \\
\text { Boxelder } \\
\text { Elm } \\
\text { Green ash } \\
\text { Cottonwood } \\
\text { Black willow } \\
\text { Hackberry } \\
\text { Black walnut } \\
\text { Other hardwoods }\end{array}$ & $\begin{array}{r}39,400 \\
5,167 \\
4,135 \\
5,992 \\
5,381 \\
5,294 \\
12,413 \\
25,900 \\
142,218 \\
11,060 \\
16,516 \\
5,131 \\
7,148 \\
\end{array}$ & $\begin{array}{r}28,691 \\
5,167 \\
4,135 \\
5,159 \\
5,381 \\
4,872 \\
11,317 \\
19,768 \\
100,334 \\
7,846 \\
15,617 \\
4,917 \\
6,372 \\
\end{array}$ & $\begin{array}{r}10,709 \\
-- \\
-- \\
833 \\
-- \\
422 \\
1,096 \\
6,132 \\
41,884 \\
3,214 \\
899 \\
214 \\
776 \\
\end{array}$ \\
\hline Total & 285,755 & 219,576 & 66,179 \\
\hline Al 1 species & 456,011 & 225,748 & 230,263 \\
\hline
\end{tabular}

Table 43.--Net volume of sawtimber on commercial forest 1 and, by species group and Forest Survey Unit, Nebraska, 1983

(In thousand board feet) $\underline{1 /}$

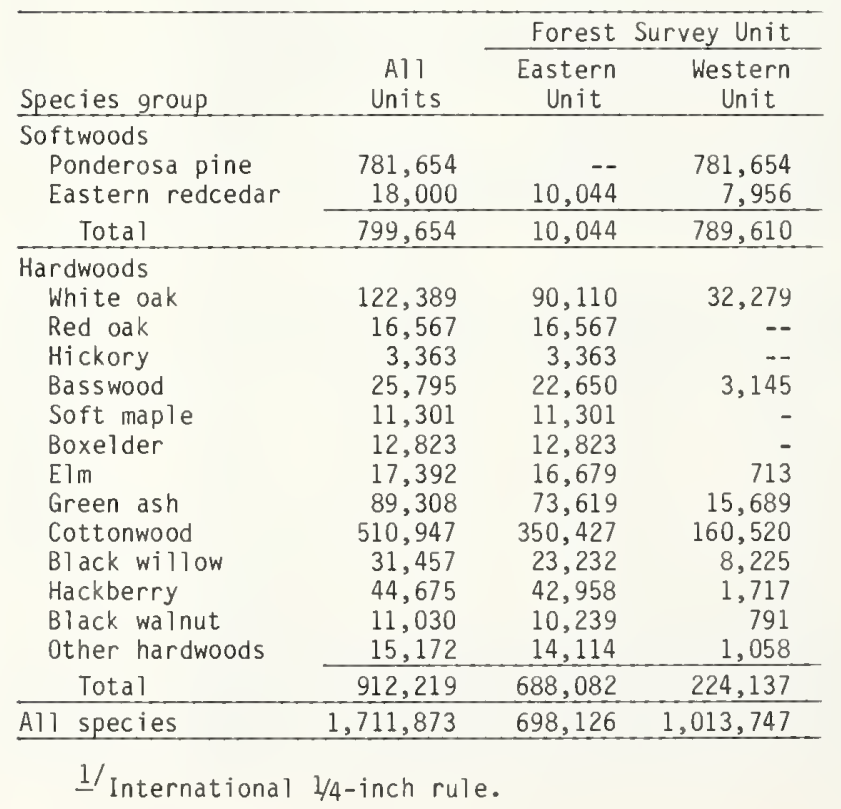




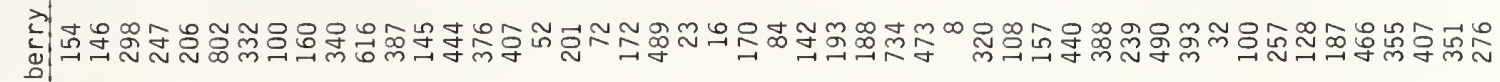

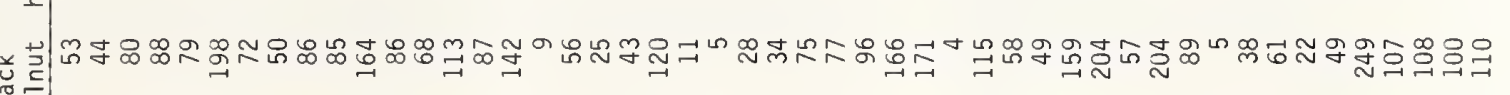
$\frac{\pi}{3}$ :

包 茰.

음

'́

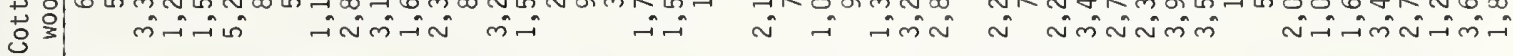

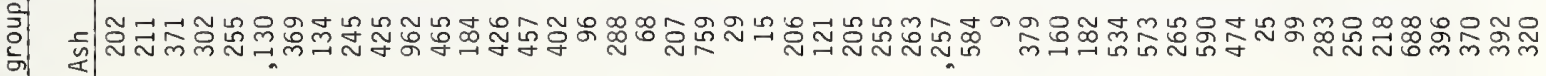

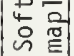

E

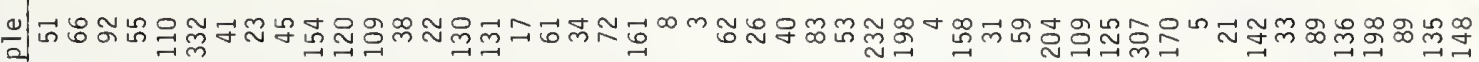

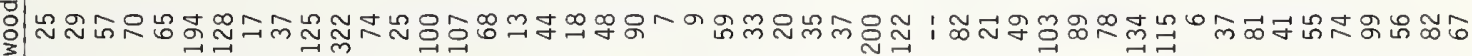
(1)

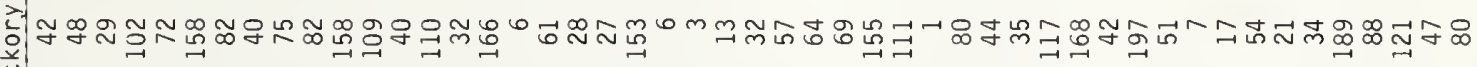
:

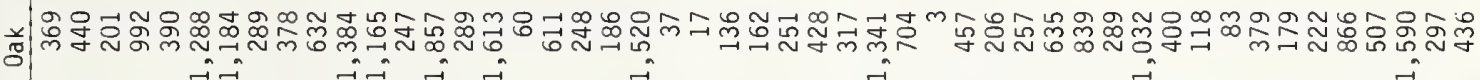

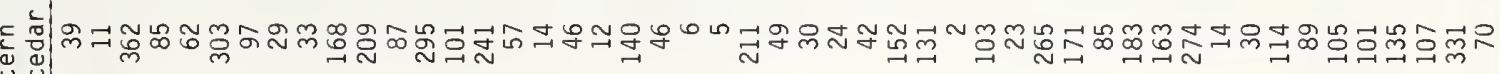

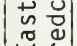

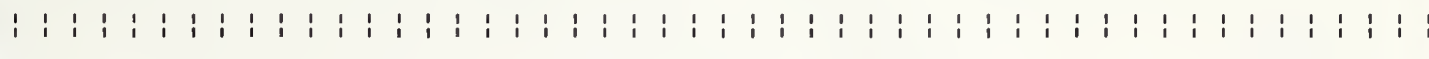
등

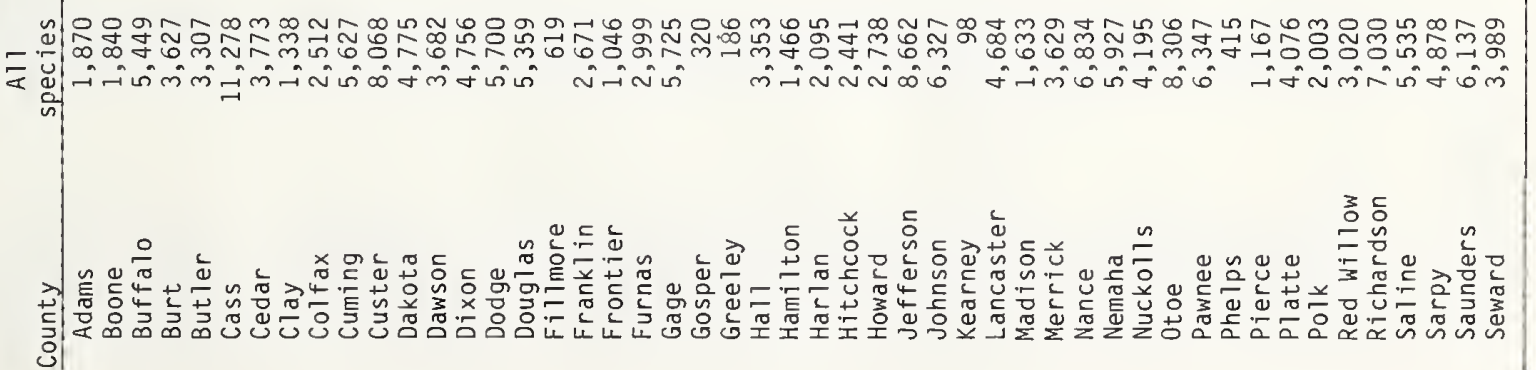




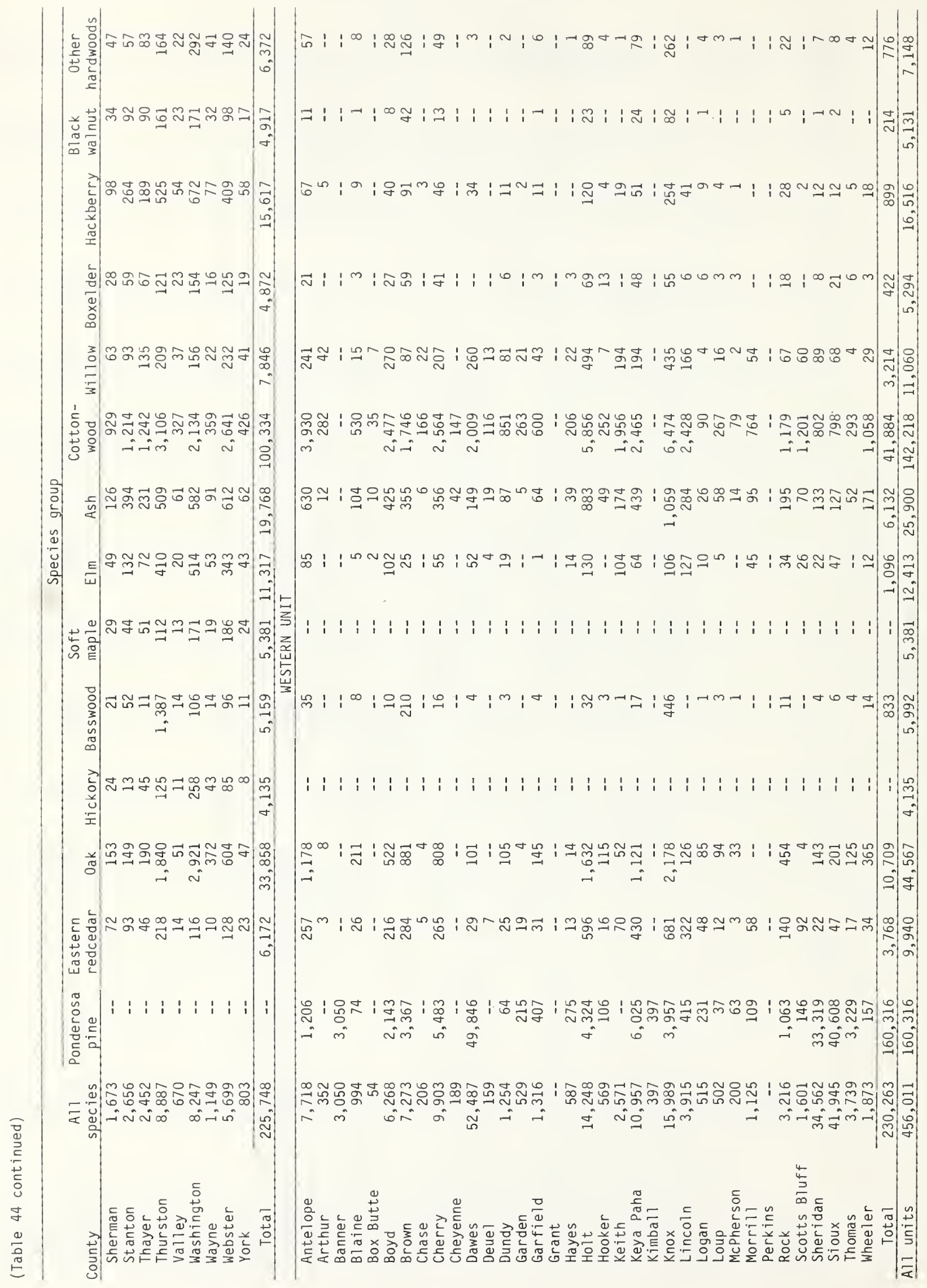




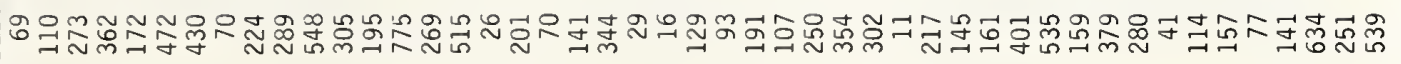

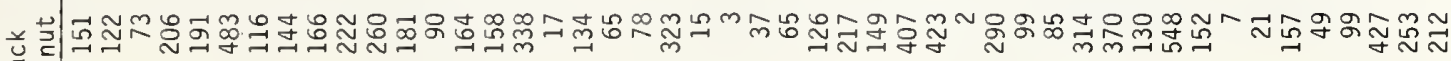
$\frac{\pi}{3}$

곤

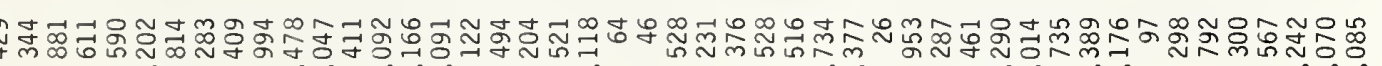

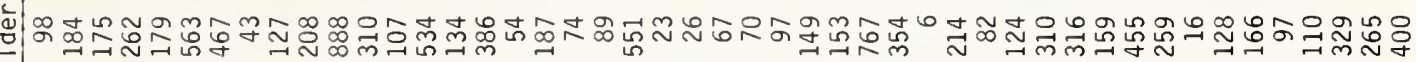

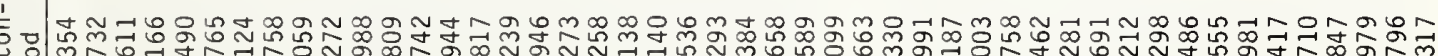

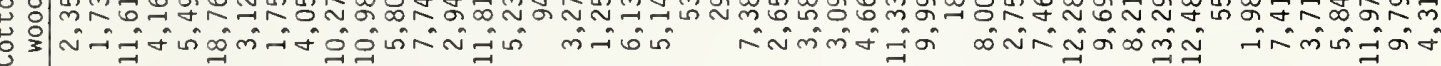

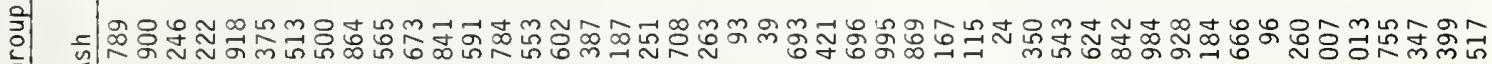
it

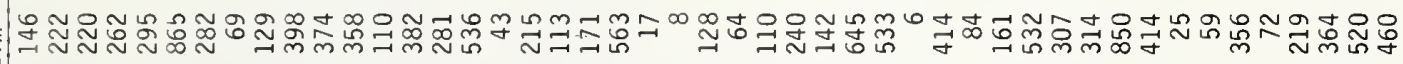

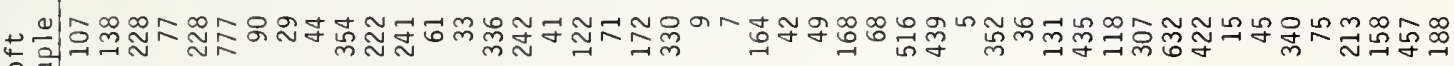
贾

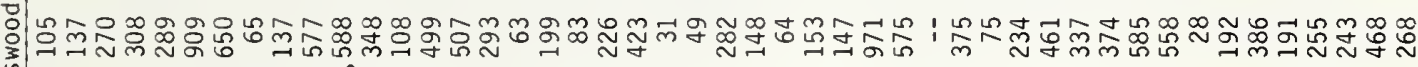
๓.

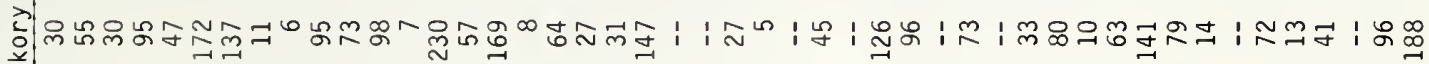
.

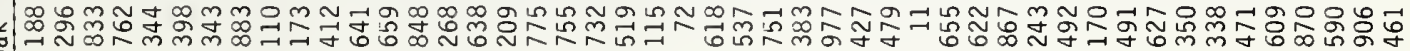

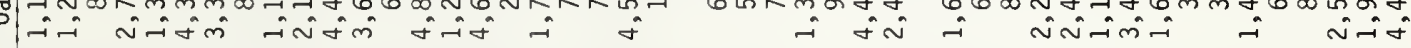

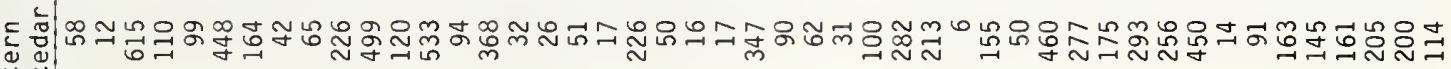

\% 这. 동

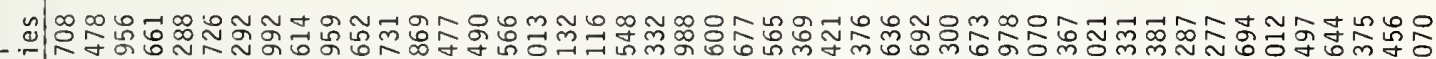
区 


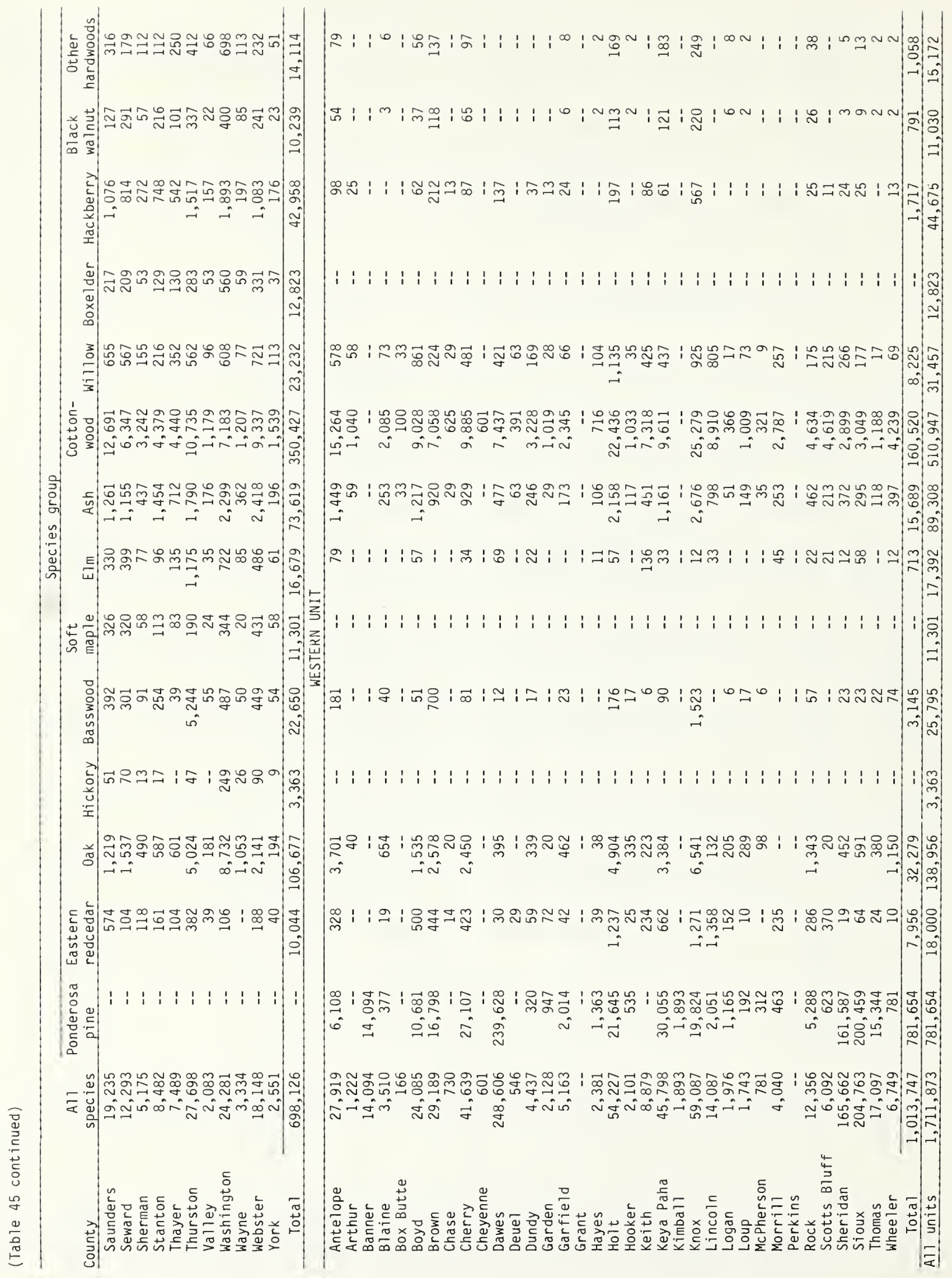




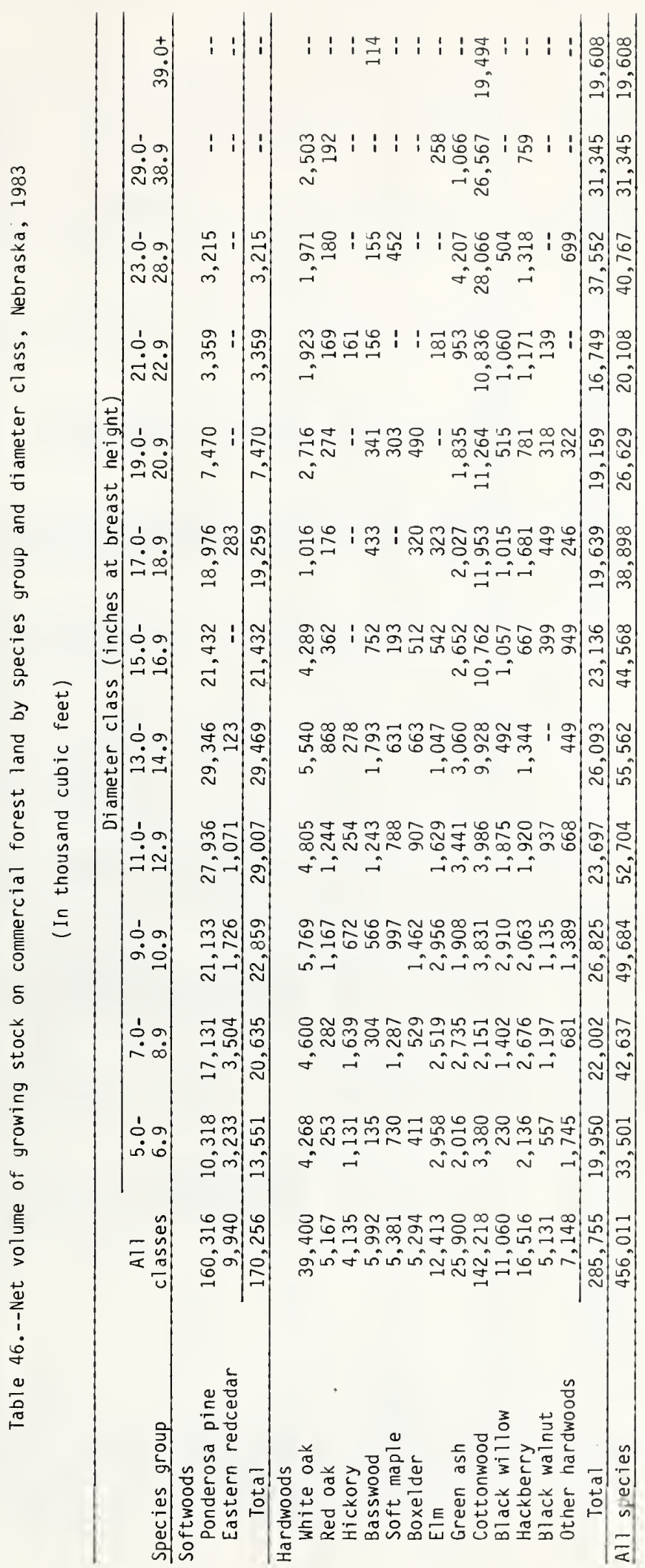




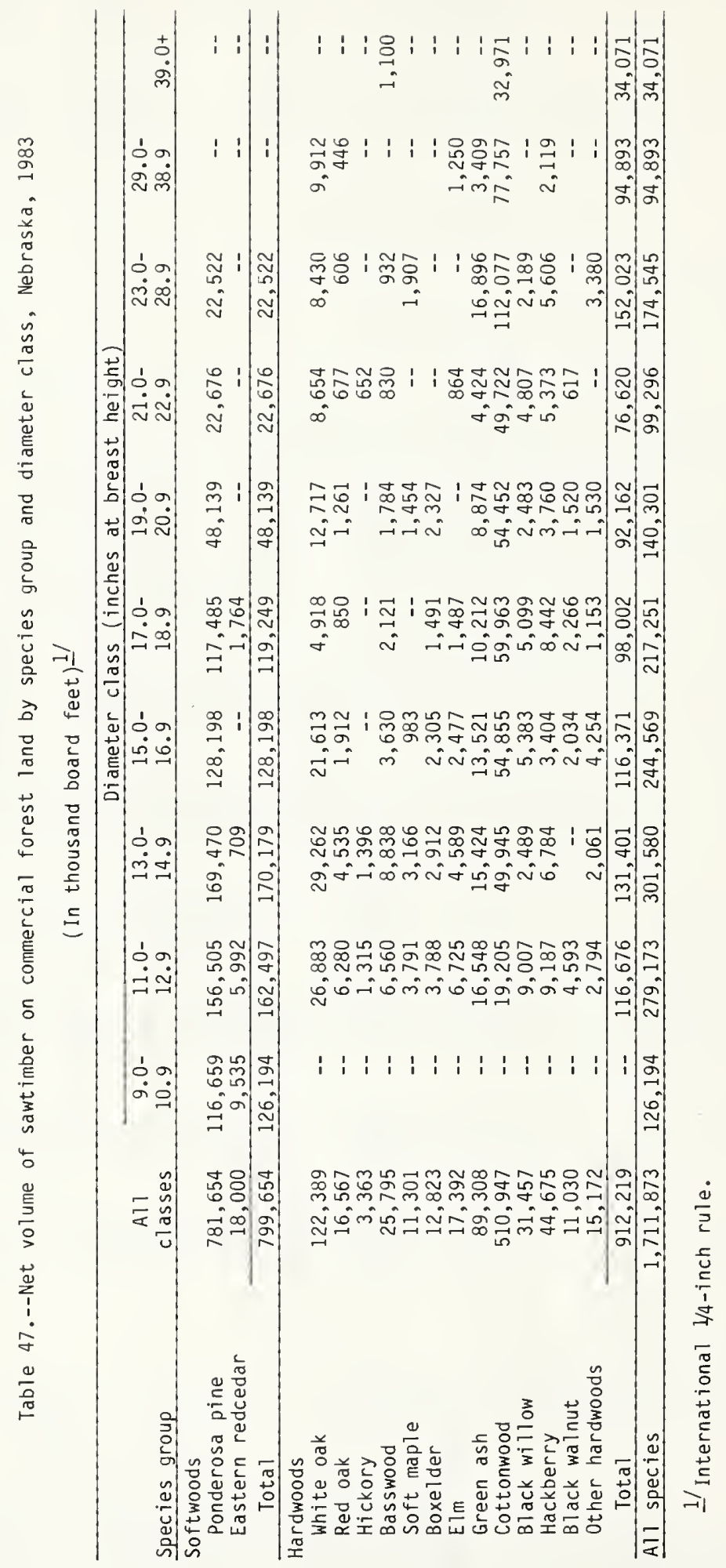


Table 48.--Net volume of growing stock on commercial forest land by species group and forest type, Nebraska, 1983

(In thousand cubic feet)

\begin{tabular}{|c|c|c|c|c|c|c|c|c|c|}
\hline \multirow[b]{2}{*}{ Species group } & \multirow[b]{2}{*}{$\begin{array}{c}\text { All } \\
\text { types }\end{array}$} & \multicolumn{8}{|c|}{ Forest type } \\
\hline & & $\begin{array}{c}\text { Ponderosa } \\
\text { pine }\end{array}$ & $\begin{array}{l}\text { Eastern } \\
\text { redcedar- } \\
\text { hardwood }\end{array}$ & $\begin{array}{c}\text { 0ak- } \\
\text { hickory }\end{array}$ & $\begin{array}{l}\text { Bur } \\
\text { oak }\end{array}$ & $\begin{array}{l}\text { Elm-ash- } \\
\text { cottonwood }\end{array}$ & Cottonwood & $\begin{array}{l}\text { Lowl and } \\
\text { plains } \\
\text { hardwoods }\end{array}$ & Nonstocked \\
\hline \multirow{3}{*}{$\begin{array}{l}\text { Softwoods } \\
\text { Ponderosa pine } \\
\text { Eastern redcedar } \\
\text { Total }\end{array}$} & & & & & & & & & \\
\hline & $\begin{array}{r}160,316 \\
9,940 \\
\end{array}$ & $\begin{array}{r}156,470 \\
-- \\
\end{array}$ & $\begin{array}{r}488 \\
6,378 \\
\end{array}$ & -- & $\begin{array}{r}1,471 \\
84 \\
\end{array}$ & $\begin{array}{l}287 \\
712 \\
\end{array}$ & 1,890 & $\begin{array}{r}1,295 \\
876 \\
\end{array}$ & $\begin{array}{r}305 \\
-- \\
\end{array}$ \\
\hline & 170,256 & 156,470 & 6,866 & -- & 1,555 & 999 & 1,890 & 2,171 & 305 \\
\hline \multicolumn{10}{|l|}{ Hardwoods } \\
\hline White oak & 39,400 & 260 & 3,057 & 6,457 & 16,504 & 2,717 & -- & 10,405 & -- \\
\hline Red oak & 5,167 & -- & 349 & 3,753 & -- & 340 & -- & 725 & -- \\
\hline Hickory & 4,135 & -- & -- & 2,670 & 653 & 161 & -- & 651 & -- \\
\hline Basswood & 5,992 & -- & 676 & 1,314 & -- & 118 & -- & 3,884 & -- \\
\hline Soft maple & 5,381 & -- & -- & - & -- & 5,237 & -- & 144 & -- \\
\hline Boxelder & 5,294 & .- & _- & -- & -- & 4,025 & -- & 1,269 & -- \\
\hline Elm & 12,413 & -- & 155 & 703 & 89 & 8,632 & 712 & 2,122 & -- \\
\hline Green ash & 25,900 & -- & 962 & 508 & 1,353 & 15,953 & 2,236 & 4,888 & -- \\
\hline Cottonwood & 142,218 & -- & 4,477 & 299 & -- & 36,968 & 96,407 & 2,993 & 1,074 \\
\hline Black willow & 11,060 & -- & 127 & -- & -- & 7,830 & 2,857 & 246 & -- \\
\hline Hackberry & 16,516 & _. & 87 & 514 & 1,203 & 2,211 & 249 & 12,252 & -- \\
\hline $\mathrm{Bl}$ ack walnut & 5,131 & -- & 139 & 1,138 & 121 & 937 & -- & 2,796 & -- \\
\hline 0ther hardwoods & 7,148 & $=-$ & 293 & 246 & 241 & 2,362 & - & 4,006 & - \\
\hline Total & 285,755 & 260 & 10,322 & 17,602 & 20,164 & 87,491 & 102,461 & 46,381 & 1,074 \\
\hline All species & 456,011 & 156,730 & 17,188 & 17,602 & 21,719 & 88,490 & 104,351 & 48,552 & $\overrightarrow{1,379}$ \\
\hline
\end{tabular}

Table 49.--Net volume of sawtimber on commercial forest land by species group and forest type, Nebraska, 1983 (In thousand board feet) 1 -

\begin{tabular}{|c|c|c|c|c|c|c|c|c|c|}
\hline \multirow[b]{2}{*}{ Species group } & \multirow[b]{2}{*}{$\begin{array}{c}\text { All } \\
\text { types }\end{array}$} & \multicolumn{8}{|c|}{ Forest type } \\
\hline & & $\begin{array}{c}\text { Ponderosa } \\
\text { pine }\end{array}$ & $\begin{array}{l}\text { Eastern } \\
\text { redcedar- } \\
\text { hardwood }\end{array}$ & $\begin{array}{c}\text { Oak- } \\
\text { hickory }\end{array}$ & $\begin{array}{l}\text { Bur } \\
\text { oak } \\
\end{array}$ & $\begin{array}{c}\text { Elm-ash- } \\
\text { cottonwood }\end{array}$ & Cottonwood & $\begin{array}{l}\text { Lowl and } \\
\text { plains } \\
\text { hardwoods }\end{array}$ & Nonstocked \\
\hline \multirow{3}{*}{$\begin{array}{l}\text { Softwoods } \\
\text { Ponderosa pine } \\
\text { Eastern redcedar } \\
\text { Total }\end{array}$} & & & & & & & & & \\
\hline & $\begin{array}{r}781,654 \\
18,000 \\
\end{array}$ & $\begin{array}{r}761,990 \\
- \\
-\end{array}$ & $\begin{array}{r}3,030 \\
12,339 \\
\end{array}$ & $=-$ & $\begin{array}{r}6,042 \\
-- \\
\end{array}$ & $\begin{array}{l}1,754 \\
1,625 \\
\end{array}$ & 2,705 & $\begin{array}{l}7,604 \\
1,331 \\
\end{array}$ & $\begin{array}{r}1,234 \\
-= \\
\end{array}$ \\
\hline & 799,654 & 761,990 & 15,369 & -- & 6,042 & 3,379 & 2,705 & 8,935 & 1,234 \\
\hline \multicolumn{10}{|l|}{ Hardwoods } \\
\hline White oak & 122,389 & -- & 14,395 & 22,227 & 42,454 & 12,355 & -- & 30,958 & -- \\
\hline Red oak & 16,567 & $\cdots$ & 1,283 & 11,075 & & 1,715 & -- & 2,494 & -- \\
\hline Hickory & 3,363 & -- & -- & 1,396 & 1,315 & 652 & -- & -- & -- \\
\hline Basswood & 25,795 & - & 2,841 & 5,033 & -- & 582 & - & 17,339 & -- \\
\hline Soft maple & 11,301 & -- & -- & -- & -- & 11,301 & -- & -- & $=$ \\
\hline Boxelder & 12,823 & -- & -- & -- & -- & 9,891 & -- & 2,932 & -- \\
\hline Elm & 17,392 & -- & -- & 896 & 365 & 11,140 & 812 & 4,179 & -- \\
\hline Green ash & 89,308 & -- & 3,367 & 1,226 & 3,256 & 56,467 & 6,463 & 18,529 & -- \\
\hline Cottonwood & 510,947 & -- & 18,698 & 850 & -- & 118,506 & 363,057 & 6,447 & 3,389 \\
\hline Black willow & 31,457 & -- & -- & -- & -- & 22,144 & 8,237 & 1,076 & -- \\
\hline Hackberry & 44,675 & -- & -- & -- & 3,187 & 7,277 & 1,252 & 32,959 & -- \\
\hline Black walnut & 11,030 & -- & 617 & 3,255 & 596 & 905 & -- & 5,657 & -- \\
\hline Other hardwoods & 15,172 & -- & -- & 1,153 & 545 & 3,999 & -- & 9,475 & -- \\
\hline Total & 912,219 & -- & 41,201 & 47,111 & 51,718 & 256,934 & 379,821 & 132,045 & 3,389 \\
\hline All species & $1,711,873$ & 761,990 & 56,570 & 47,111 & 57,760 & 260,313 & 382,526 & 140,980 & 4,623 \\
\hline
\end{tabular}


Table 50.--Net volume of growing stock on commercial forest land by species group and ownership class, Nebraska, 1983

(In thousand cubic feet)

\begin{tabular}{|c|c|c|c|c|c|c|c|c|}
\hline \multirow[b]{2}{*}{ Species group } & \multirow[b]{2}{*}{$\begin{array}{c}\text { All } \\
\text { classes }\end{array}$} & \multicolumn{7}{|c|}{ Ownership class } \\
\hline & & $\begin{array}{c}\text { National } \\
\text { Forest }\end{array}$ & State & $\begin{array}{c}\text { County and } \\
\text { municipal }\end{array}$ & Indian & Farmer & $\begin{array}{c}\text { Misc. } \\
\text { private } \\
\text { coporation }\end{array}$ & $\begin{array}{l}\text { Misc. } \\
\text { private } \\
\text { individual }\end{array}$ \\
\hline \multirow{4}{*}{$\begin{array}{l}\text { Softwoods } \\
\text { Ponderosa pine } \\
\text { Eastern redcedar } \\
\text { Total }\end{array}$} & & & & & & & & \\
\hline & 160,316 & 37,438 & 13,721 & -- & -- & 97,160 & -- & \\
\hline & 9,940 & -- & 2,105 & -- & $=$ & 3,729 & 470 & 3,636 \\
\hline & 170,256 & 37,438 & 15,826 & - & - & 100,889 & 470 & 15,633 \\
\hline \multicolumn{9}{|l|}{ Hardwoods } \\
\hline White oak & 39,400 & -- & - & -- & 1,441 & 28,721 & 1,238 & 8,000 \\
\hline Red oak & 5,167 & -- & -- & -- & 556 & 1,414 & - & 3,197 \\
\hline Hickory & 4,135 & -- & -- & -- & -- & 1,489 & 1,161 & 1,485 \\
\hline Basswood & 5,992 & -- & $-\infty$ & -- & 860 & 5,132 & - & -- \\
\hline Soft maple & 5,381 & -- & -- & -- & -- & 5,381 & -- & -- \\
\hline Boxelder & 5,294 & -- & -- & -- & - & 5,294 & - & -- \\
\hline Elm & 12,413 & -- & -- & 118 & 207 & 11,255 & -- & 833 \\
\hline Green ash & 25,900 & -- & -- & 1,343 & -- & 20,644 & 231 & 3,682 \\
\hline Cottonwood & 142,218 & -- & 9,678 & -- & 263 & 116,011 & 2,735 & 13,531 \\
\hline Black willow & 11,060 & -- & -- & -- & $\ldots$ & 7,576 & 997 & 2,487 \\
\hline Hackberry & 16,516 & - & -- & -- & 151 & 15,009 & -- & 1,356 \\
\hline Black walnut & 5,131 & -- & -- & -- & -- & 3,015 & -- & 2,116 \\
\hline Other hardwoods & 7,148 & -- & -- & $\therefore$ & $=$ & 5,533 & 241 & 1,374 \\
\hline Total & 285,755 & -- & 9,678 & 1,461 & 3,478 & 226,474 & 6,603 & 38,061 \\
\hline All species & 456,011 & 37,438 & 25,504 & 1,461 & 3,478 & 327,363 & 7,073 & 53,694 \\
\hline
\end{tabular}

Table 51.--Net volume of sawtimber on commercial forest land by species group and ownership class, Nebraska, 1983

(In thousand board feet) 1 /

\begin{tabular}{|c|c|c|c|c|c|c|c|c|}
\hline \multirow[b]{2}{*}{ Species group } & \multirow[b]{2}{*}{$\begin{array}{c}\text { Al1 } \\
\text { classes }\end{array}$} & \multicolumn{7}{|c|}{ Ownership class } \\
\hline & & $\begin{array}{l}\text { National } \\
\text { Forest }\end{array}$ & State & $\begin{array}{l}\text { County and } \\
\text { municipal }\end{array}$ & Indian & Farmer & $\begin{array}{c}\text { Misc. } \\
\text { private } \\
\text { coporation }\end{array}$ & $\begin{array}{c}\text { Misc. } \\
\text { private } \\
\text { individual }\end{array}$ \\
\hline \multirow{4}{*}{$\begin{array}{l}\text { Softwoods } \\
\text { Ponderosa pine } \\
\text { Eastern redcedar } \\
\text { Total }\end{array}$} & & & & & & & & \\
\hline & 781,654 & 173,169 & 79,275 & - & - & 475,849 & -- & 53,361 \\
\hline & 18,000 & $=$ & 4,011 & -- & -- & 5,984 & 1,932 & 6,073 \\
\hline & 799,654 & 173,169 & 83,286 & -- & - & 481,833 & 1,932 & 59,434 \\
\hline \multicolumn{9}{|l|}{ Hardwoods } \\
\hline White oak & 122,389 & -- & -- & -- & 1,587 & 95,487 & 4,762 & 20,553 \\
\hline Red oak & 16,567 & -- & -- & -- & 1,261 & 5,492 & -- & 9,814 \\
\hline Hickory & 3,363 & -- & -- & -- & - & 2,048 & -- & 1,315 \\
\hline Basswood & 25,795 & -- & -- & -- & 2,833 & 22,962 & -- & - \\
\hline Soft maple & 11,301 & -- & $=-$ & - & -. & 11,301 & -- & -- \\
\hline Boxelder & 12,823 & -- & -- & -- & -- & 12,823 & -- & -- \\
\hline $\mathrm{E} \operatorname{lm}$ & 17,392 & -- & -- & -- & 896 & 16,131 & -- & 365 \\
\hline Green ash & 89,308 & -- & -- & 4,410 & -- & 71,487 & 84 & 13,327 \\
\hline Cottonwood & 510,947 & -- & 46,308 & -- & - & 404,406 & 10,391 & 49,842 \\
\hline Black willow & 31,457 & -- & -- & -- & -- & 26,757 & 2,894 & 1,806 \\
\hline Hackberry & 44,675 & -- & -- & -- & -- & 42,596 & -- & 2,079 \\
\hline Black walnut & 11,030 & -- & -- & -- & -- & 3,856 & -- & 7,174 \\
\hline 0ther hardwoods & 15,172 & -- & -- & $=$ & -- & 11,038 & 545 & 3,589 \\
\hline Total & 912,219 & -- & 46,308 & 4,410 & 6,577 & 726,384 & 18,676 & 109,864 \\
\hline A11 species & $1,711,873$ & 173,169 & 129,594 & 4,410 & 6,577 & $1,208,217$ & 20,608 & 169,298 \\
\hline
\end{tabular}

1/ International 1/4-inch rule. 

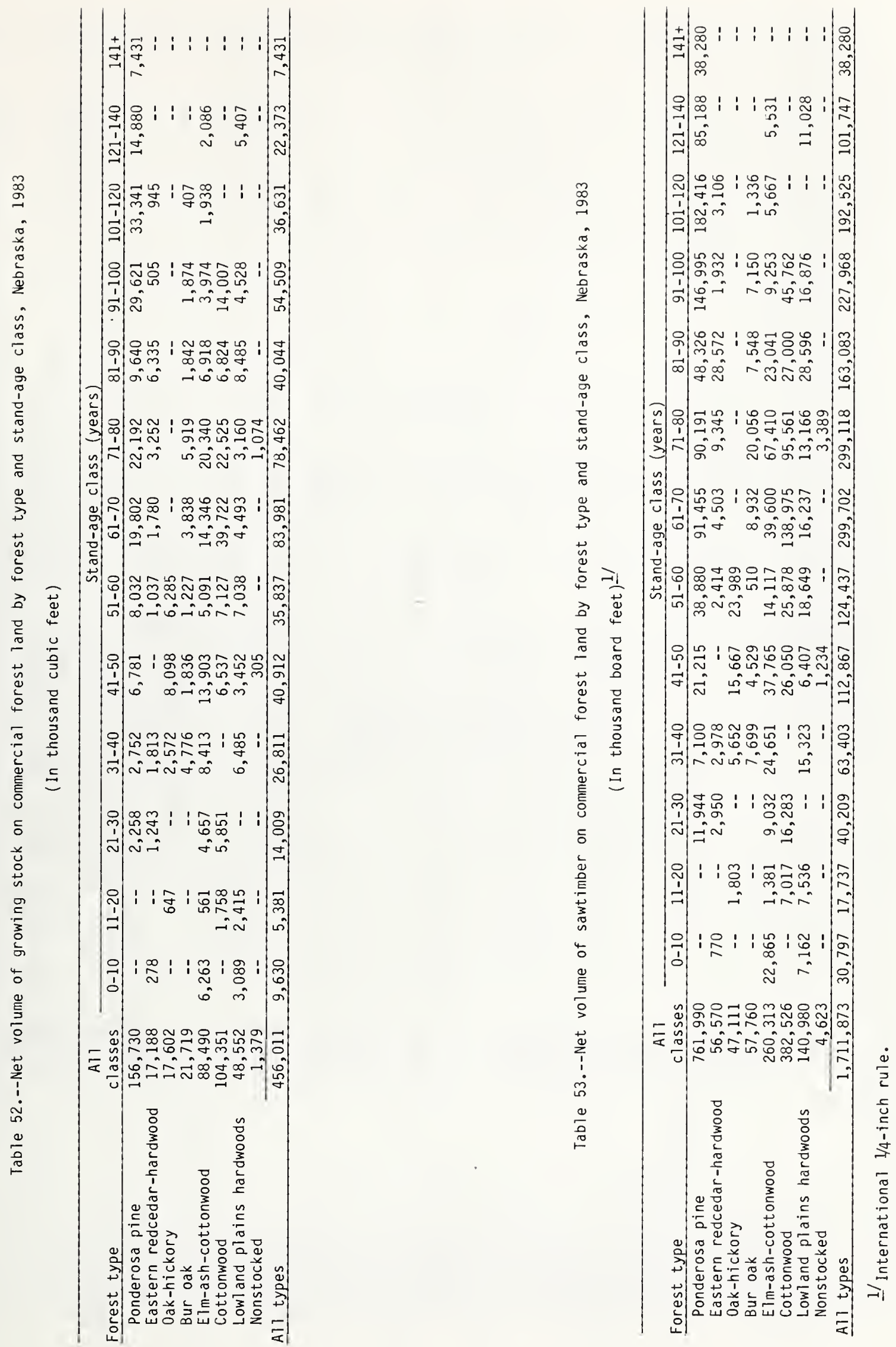


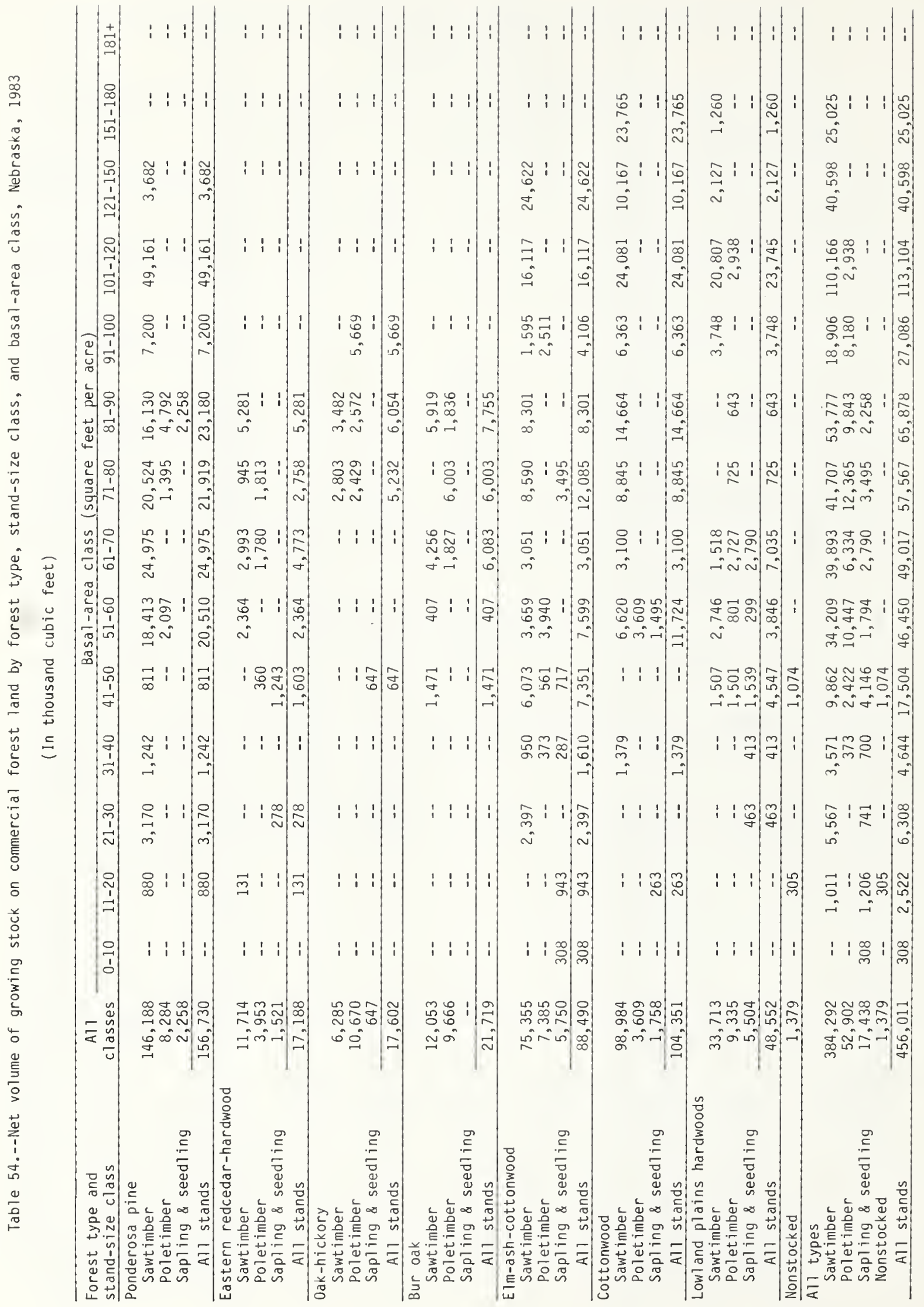




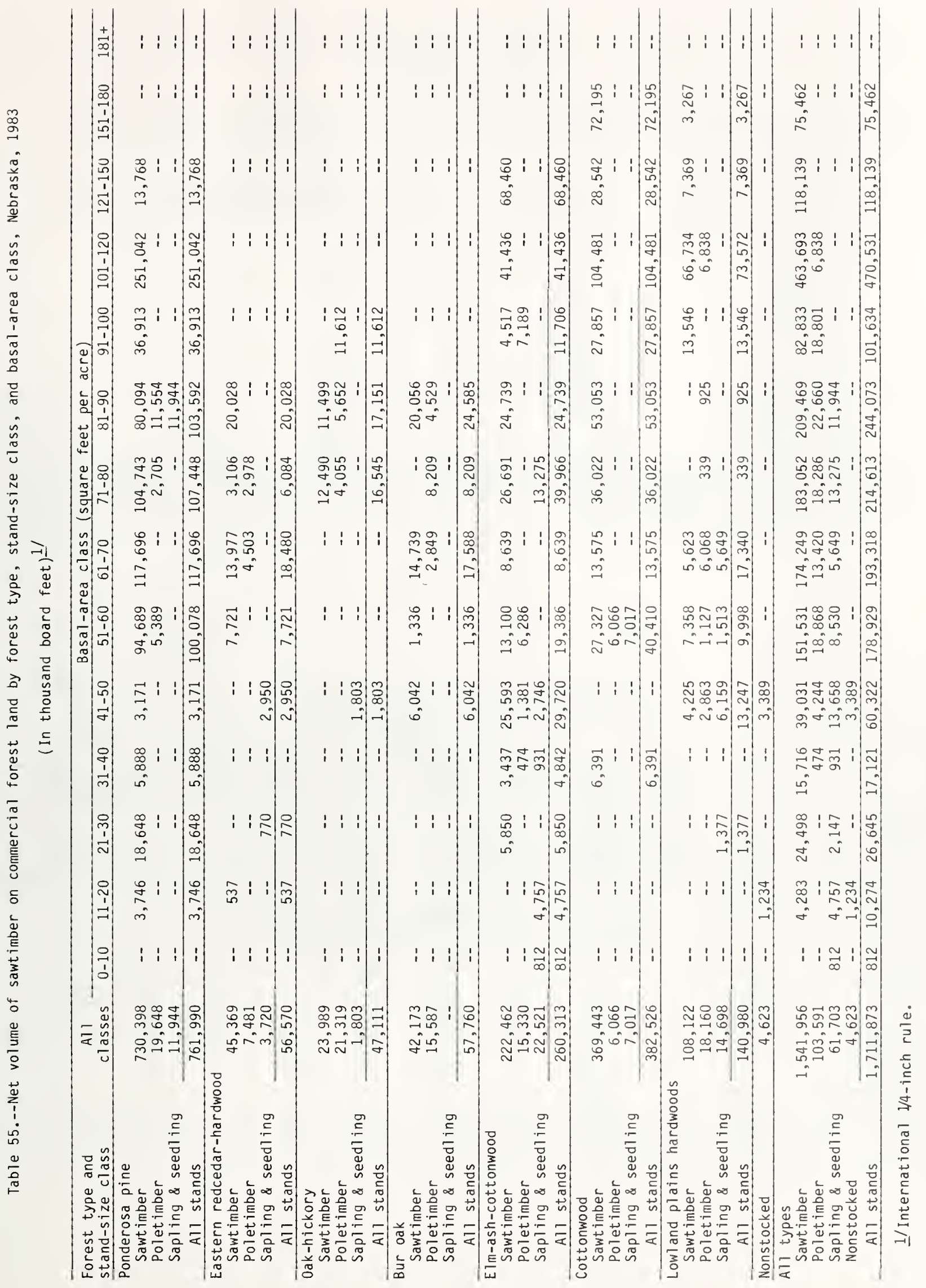


Table 56.--Net volume of sawtimber on commercial forest land by species group and log-grade, Nebraska, 1983

(In thousand board feet) 1 '

\begin{tabular}{|c|c|c|c|c|c|}
\hline \multirow[b]{2}{*}{ Species group } & \multirow{2}{*}{$\begin{array}{l}\text { All } \\
\text { grades }\end{array}$} & \multicolumn{4}{|c|}{ Log grade } \\
\hline & & 1 & 2 & 3 & Tie and timber \\
\hline \multicolumn{6}{|l|}{ Softwoods } \\
\hline Ponderosa pine & 781,654 & 3,844 & 31,988 & 745,822 & -- \\
\hline Eastern redcedar & 18,000 & $\ldots$ & $=$ & 18,000 & -- \\
\hline Total & 799,654 & 3,844 & 31,988 & 763,822 & -- \\
\hline \multicolumn{6}{|l|}{ Hardwoods } \\
\hline White oak & 122,389 & 3,706 & 37,872 & 61,503 & 19,308 \\
\hline Red oak & 16,567 & -- & 2,767 & 13,800 & -- \\
\hline Hickory & 3,363 & -- & 1,306 & 2,057 & -- \\
\hline Basswood & 25,795 & 1,677 & 5,738 & 18,380 & -- \\
\hline Soft maple & 11,301 & -- & -- & 10,262 & 1,039 \\
\hline Boxelder & 12,823 & -- & 563 & 8,850 & 3,410 \\
\hline E $1 \mathrm{~m}$ & 17,392 & 1,859 & 5,291 & 5,927 & 4,315 \\
\hline Green ash & 89,308 & 4,951 & 26,448 & 47,826 & 10,083 \\
\hline Cottonwood & 510,947 & 203,039 & 157,036 & 130,074 & 20,798 \\
\hline Black willow & 31,457 & 2,156 & 5,306 & 19,121 & 4,874 \\
\hline Hackberry & 44,675 & -- & 25,666 & 19,009 & -- \\
\hline Black walnut & 11,030 & 195 & 3,248 & 7,587 & -- \\
\hline other hardwoods & 15,172 & -- & 3,656 & 10,749 & 767 \\
\hline Total & 912,219 & 217,583 & 274,897 & 355,145 & 64,594 \\
\hline All species & $1,711,873$ & 221,427 & 306,885 & $1,118,967$ & 64,594 \\
\hline
\end{tabular}

1/International $1 / 4$-inch rule.

Table 57.--Net volume of short-log trees on commercial forest land by species group and diameter class, Nebraska, 1983 (In thousand cubic feet)

\begin{tabular}{|c|c|c|c|c|c|c|c|c|c|c|c|}
\hline & & & & & Diameter & class (i & hes at & ast he i & ght) & & \\
\hline Species group & $\begin{array}{c}\text { Al1 } \\
\text { classes }\end{array}$ & $\begin{array}{r}9.0- \\
10.9\end{array}$ & $\begin{array}{l}11.0- \\
12.9\end{array}$ & $\begin{array}{l}13.0- \\
14.9\end{array}$ & $\begin{array}{l}15.0- \\
16.9\end{array}$ & $\begin{array}{l}17.0- \\
18.9\end{array}$ & $\begin{array}{l}19.0- \\
20.9\end{array}$ & $\begin{array}{l}21.0- \\
22.9\end{array}$ & $\begin{array}{l}23.0- \\
28.9\end{array}$ & $\begin{array}{l}29.0- \\
38.9\end{array}$ & $39.0+$ \\
\hline Softwoods & & & & & & & & & & & \\
\hline Ponderosa pine & 2,198 & 846 & 1,002 & 162 & 129 & 59 & - & $=$ & - & - & -- \\
\hline Eastern redcedar & 487 & 233 & 178 & 76 & - & - & -- & -- & -- & -- & -- \\
\hline Total & 2,685 & 1,079 & 1,180 & 238 & 129 & 59 & -- & - & -- & - & -- \\
\hline Hardwoods & & & & & & & & & & & \\
\hline White oak & 4,657 & - & 1,170 & 844 & 857 & 387 & 725 & 404 & 270 & - & -- \\
\hline Red oak & 555 & $=-$ & 171 & 205 & 179 & -. & -- & -- & -- & -- & -- \\
\hline Hickory & - & - & $\ldots$ & -- & - & -- & -- & -- & - & -- & -- \\
\hline Basswood & 321 & - & 267 & -- & -- & 54 & -- & -- & -- & -- & -- \\
\hline Soft maple & 648 & $=-$ & -- & 445 & 95 & -- & -- & -- & 108 & -- & -- \\
\hline Boxelder & 1,271 & -- & 447 & -- & 387 & 187 & 250 & -- & -- & -- & -- \\
\hline Elm & 639 & - & 105 & 136 & 249 & -- & -- & -- & - & 149 & -- \\
\hline Green ash & 1,731 & -- & 577 & 649 & 280 & 225 & -- & -- & -- & - & -- \\
\hline Cottonwood & 4,112 & -- & 456 & 794 & -- & 480 & 367 & 136 & 381 & 867 & 631 \\
\hline Black willow & 424 & -- & 161 & -- & 169 & 94 & -- & - & - & -- & - \\
\hline Hackberry & 1,404 & -- & 481 & -. & 159 & -- & 173 & -- & 591 & -- & -- \\
\hline Black walnut & 263 & -- & 157 & - & 106 & $\cdots$ & -- & -- & -- & -- & -- \\
\hline Other hardwoods & 775 & -- & 403 & 178 & -- & 89 & -- & -- & 105 & -- & -- \\
\hline Total & 16,800 & $\because-$ & 4,395 & 3,251 & 2,481 & 1,516 & 1,515 & 540 & 1,455 & 1,016 & 631 \\
\hline All species & 19,485 & 1,079 & 5,575 & 3,489 & 2,610 & 1,575 & 1,515 & 540 & 1,455 & 1,016 & 631 \\
\hline
\end{tabular}


Table 58.--Net volume of short-log trees on commercial forest land by species group and diameter class, Nebraska, 1983

(In thousand board feet) $\underline{1 /}$

\begin{tabular}{|c|c|c|c|c|c|c|c|c|c|c|c|}
\hline \multirow[b]{2}{*}{ Species group } & \multirow[b]{2}{*}{$\begin{array}{c}\text { All } \\
\text { classes } \\
\end{array}$} & \multicolumn{10}{|c|}{ Diameter class (inches at breast height) } \\
\hline & & $\begin{array}{c}9.0- \\
10.9 \\
\end{array}$ & $\begin{array}{l}11.0- \\
12.9\end{array}$ & $\begin{array}{l}13.0- \\
14.9\end{array}$ & $\begin{array}{l}15.0- \\
16.9\end{array}$ & $\begin{array}{l}17.0- \\
18.9\end{array}$ & $\begin{array}{l}19.0- \\
20.9\end{array}$ & $\begin{array}{l}21.0- \\
22.9 \\
\end{array}$ & $\begin{array}{l}23.0- \\
28.9 \\
\end{array}$ & $\begin{array}{l}29.0- \\
38.9 \\
\end{array}$ & $39.0+$ \\
\hline $\begin{array}{l}\text { Softwoods } \\
\text { Ponderosa pine } \\
\text { Eastern redcedar }\end{array}$ & $\begin{array}{r}25,586 \\
5,278 \\
\end{array}$ & $\begin{array}{l}7,524 \\
2,217 \\
\end{array}$ & $\begin{array}{r}12,011 \\
1,993 \\
\end{array}$ & $\begin{array}{l}2,386 \\
1,068\end{array}$ & $\begin{array}{r}2,337 \\
-- \\
\end{array}$ & $\begin{array}{r}1,328 \\
--\end{array}$ & $\begin{array}{l}-- \\
--\end{array}$ & $\begin{array}{l}-- \\
--\end{array}$ & $\begin{array}{l}-- \\
--\end{array}$ & $\begin{array}{l}-- \\
-\end{array}$ & -- \\
\hline Total & 30,864 & 9,741 & 14,004 & 3,454 & 2,337 & 1,328 & -- & -- & $=$ & $\because-$ & -- \\
\hline $\begin{array}{l}\text { Hardwoods } \\
\text { White oak } \\
\text { Red oak } \\
\text { Hickory } \\
\text { Basswood } \\
\text { Soft maple } \\
\text { Boxelder } \\
\text { Elm } \\
\text { Green ash } \\
\text { Cottonwood } \\
\text { Black willow } \\
\text { Hackberry } \\
\text { Black walnut } \\
\text { Other hardwoods }\end{array}$ & $\begin{array}{r}14,972 \\
1,877 \\
-- \\
1,119 \\
1,978 \\
3,940 \\
1,839 \\
5,396 \\
9,983 \\
1,326 \\
3,903 \\
820 \\
2,337 \\
\end{array}$ & $\begin{array}{l}-- \\
-- \\
-- \\
-- \\
-- \\
-- \\
-- \\
-- \\
-- \\
-- \\
-- \\
--\end{array}$ & $\begin{array}{r}5,563 \\
571 \\
-- \\
927 \\
-- \\
1,385 \\
318 \\
1,744 \\
1,405 \\
493 \\
1,446 \\
480 \\
1,243 \\
\end{array}$ & $\begin{array}{r}3,193 \\
695 \\
-- \\
-- \\
1,416 \\
-- \\
433 \\
2,067 \\
2,522 \\
-- \\
-- \\
-- \\
566 \\
\end{array}$ & $\begin{array}{r}2,620 \\
611 \\
-- \\
-- \\
304 \\
1,234 \\
794 \\
890 \\
-- \\
537 \\
506 \\
340 \\
-- \\
\end{array}$ & $\begin{array}{r}967 \\
-- \\
-- \\
192 \\
-- \\
583 \\
-- \\
695 \\
1,500 \\
296 \\
-- \\
-- \\
281 \\
\end{array}$ & $\begin{array}{r}1,530 \\
-- \\
-- \\
-- \\
-- \\
738 \\
-- \\
-- \\
1,088 \\
-- \\
517 \\
-- \\
-- \\
\end{array}$ & $\begin{array}{l}737 \\
-- \\
-- \\
-- \\
-- \\
-- \\
-- \\
-- \\
383 \\
-- \\
-- \\
-- \\
- \\
\end{array}$ & $\begin{array}{r}362 \\
-- \\
-- \\
-- \\
258 \\
-- \\
-- \\
-- \\
986 \\
-- \\
1,434 \\
-- \\
247 \\
\end{array}$ & $\begin{array}{r}-- \\
-- \\
-- \\
-- \\
-- \\
-- \\
294 \\
-- \\
1,459 \\
-- \\
-- \\
-- \\
-- \\
\end{array}$ & $\begin{array}{c}-- \\
-- \\
-- \\
-- \\
-- \\
-- \\
-- \\
-- \\
640 \\
-- \\
-- \\
-- \\
--\end{array}$ \\
\hline Total & 49,490 & $\therefore$ & 15,575 & 10,892 & 7,836 & 4,514 & 3,873 & 1,120 & 3,287 & 1,753 & 640 \\
\hline Al1 species & 80,354 & 9,741 & 29,579 & 14,346 & 10,173 & 5,842 & 3,873 & 1,120 & 3,287 & 1,753 & 640 \\
\hline
\end{tabular}

1/International $1 / 4$-inch rule.

Table 59.---Net annual growth of growing stock

on commercial forest land by softwoods and

hardwoods, Nebraska, 1955 and 1982

(In thousand cubic feet)

\begin{tabular}{lrr}
\hline Species & 1955 & 1982 \\
\hline Softwoods & 3,400 & 6,038 \\
Hardwoods & 5,100 & 5,061 \\
\hline All species & 8,500 & 11,099 \\
\hline
\end{tabular}

1/Figures have been adjusted from those published after the 1955 survey to conform to 1982 volumes because of changes in survey definitions and procedures. 
Table 60.--Net annual growth of growing stock on commercial forest land by species group and Forest Survey Unit, Nebraska, 1982

(In thousand cubic feet)

\begin{tabular}{|c|c|c|c|}
\hline \multirow[b]{2}{*}{ Species group } & \multirow[b]{2}{*}{$\begin{array}{c}\text { All } \\
\text { Units }\end{array}$} & \multicolumn{2}{|c|}{ Forest Survey Unit } \\
\hline & & $\begin{array}{c}\text { Eastern } \\
\text { Unit }\end{array}$ & $\begin{array}{c}\text { Western } \\
\text { Unit }\end{array}$ \\
\hline $\begin{array}{l}\text { Softwoods } \\
\text { Ponderosa pine } \\
\text { Eastern redcedar }\end{array}$ & $\begin{array}{r}5,415 \\
623 \\
\end{array}$ & 489 & $\begin{array}{r}5,415 \\
134 \\
\end{array}$ \\
\hline Total & 6,038 & 489 & 5,549 \\
\hline $\begin{array}{l}\text { Hardwoods } \\
\text { White oak } \\
\text { Red oak } \\
\text { Hickory } \\
\text { Basswood } \\
\text { Soft maple } \\
\text { Boxelder } \\
\text { Elm } \\
\text { Green ash } \\
\text { Cottonwood } \\
\text { Black willow } \\
\text { Hackberry } \\
\text { Black walnut } \\
\text { Other hardwoods }\end{array}$ & $\begin{array}{r}718 \\
70 \\
125 \\
112 \\
199 \\
180 \\
288 \\
408 \\
1,703 \\
137 \\
665 \\
135 \\
321 \\
\end{array}$ & $\begin{array}{r}494 \\
70 \\
125 \\
96 \\
199 \\
168 \\
368 \\
264 \\
1,278 \\
91 \\
631 \\
129 \\
289 \\
\end{array}$ & $\begin{array}{r}224 \\
-- \\
-- \\
16 \\
-- \\
12 \\
-80 \\
144 \\
425 \\
46 \\
34 \\
6 \\
32 \\
\end{array}$ \\
\hline Total & 5,061 & 4,202 & 859 \\
\hline All species & 11,099 & 4,691 & 6,408 \\
\hline
\end{tabular}

Table 61.--Net annual growth of sawtimber on commercial forest land by species group and Forest Survey Unit, Nebraska, 1982

(In thousand board feet) 1 /

\begin{tabular}{|c|c|c|c|}
\hline & & Forest & vey Unit \\
\hline Species group & $\begin{array}{l}\text { All } \\
\text { Units }\end{array}$ & $\begin{array}{c}\text { Eastern } \\
\text { Unit }\end{array}$ & $\begin{array}{c}\text { Western } \\
\text { Unit }\end{array}$ \\
\hline Softwoods & & & \\
\hline $\begin{array}{l}\text { Ponderosa pine } \\
\text { Eastern redcedar }\end{array}$ & $\begin{array}{r}27,197 \\
502 \\
\end{array}$ & 333 & $\begin{array}{r}27,197 \\
169 \\
\end{array}$ \\
\hline Total & 27,699 & 333 & 27,366 \\
\hline Hardwoods & & & \\
\hline White oak & 3,543 & 1,953 & 1,590 \\
\hline Red oak & 96 & 96 & - \\
\hline Hickory & 28 & 28 & -- \\
\hline Basswood & 1,015 & 976 & 39 \\
\hline Soft maple & 386 & 386 & - \\
\hline Boxelder & 369 & 369 & -- \\
\hline Elm & 316 & 769 & -453 \\
\hline Green ash & 1,095 & 757 & 338 \\
\hline Cottonwood & 4,690 & 4,320 & 370 \\
\hline Black willow & 263 & 61 & 202 \\
\hline Hackberry & 2,571 & 2,512 & 59 \\
\hline Black walnut & 236 & 217 & 19 \\
\hline Other hardwoods & 422 & 383 & 39 \\
\hline Total. & 15,030 & 12,827 & 2,203 \\
\hline A11 species & 42,729 & 13,160 & 29,569 \\
\hline
\end{tabular}

Table 62.--Net annual growth of growing stock on commercial forest land by species group and ownership class, Nebraska, 1983

(In thousand cubic feet)

\begin{tabular}{|c|c|c|c|c|c|c|c|c|c|}
\hline \multirow[b]{2}{*}{ Species group } & & \multicolumn{8}{|c|}{ Ownership class } \\
\hline & $\begin{array}{c}\text { All } \\
\text { classes }\end{array}$ & $\begin{array}{c}\text { National } \\
\text { Forest }\end{array}$ & $\begin{array}{l}\text { Misc. } \\
\text { federal }\end{array}$ & State & $\begin{array}{l}\text { County and } \\
\text { municipal }\end{array}$ & Indian & Farmer & $\begin{array}{l}\text { Misc. } \\
\text { private } \\
\text { coporation }\end{array}$ & $\begin{array}{l}\text { Misc. } \\
\text { private } \\
\text { individual }\end{array}$ \\
\hline Ponderosa pine & 5,415 & 1,479 & -- & 350 & -- & -- & 3,281 & -- & 305 \\
\hline Eastern redcedar & 623 & - & -- & 30 & -- & -- & 349 & 9 & 235 \\
\hline Total & 6,038 & 1,479 & -- & 380 & -- & -- & 3,630 & 9 & 540 \\
\hline Hickory & 125 & -- & -- & -- & -- & - & 54 & 33 & 38 \\
\hline Basswood & 112 & -- & - & -- & -- & 24 & 88 & - & -- \\
\hline Soft maple & 199 & -- & -- & -- & -- & - & 199 & -- & - \\
\hline Boxelder & 180 & -- & -- & -- & -- & -- & 180 & -- & -- \\
\hline Elm & 288 & -- & -- & -- & 2 & -59 & 334 & -- & 11 \\
\hline Green ash & 408 & -- & -- & -- & 19 & -72 & 394 & 6 & 61 \\
\hline Total & 5,061 & $\ldots$ & $\cdots$ & 158 & 21 & -46 & 4,138 & 36 & 754 \\
\hline All species & 11,099 & 1,479 & -- & 538 & 21 & -46 & 7,768 & 45 & 1,294 \\
\hline
\end{tabular}


Table 63.--Net annual growth of sawtimber on commercial forest land by species group and ownership class, Nebraska, 1983

(In thousand board feet) 1 /

\begin{tabular}{|c|c|c|c|c|c|c|c|c|c|}
\hline \multirow[b]{2}{*}{ Species group } & \multirow[b]{2}{*}{$\begin{array}{c}\text { All } \\
\text { classes } \\
\end{array}$} & \multicolumn{8}{|c|}{ Ownership class } \\
\hline & & $\begin{array}{c}\text { National } \\
\text { Forest } \\
\end{array}$ & $\begin{array}{l}\text { Misc. } \\
\text { federal }\end{array}$ & State & $\begin{array}{r}\text { County and } \\
\text { municipal }\end{array}$ & Indian & Farmer & $\begin{array}{l}\text { Misc. } \\
\text { private } \\
\text { coporation }\end{array}$ & $\begin{array}{c}\text { Misc. } \\
\text { private } \\
\text { individual }\end{array}$ \\
\hline \multirow{4}{*}{$\begin{array}{l}\text { Softwoods } \\
\text { Ponderosa pine } \\
\text { Eastern redcedar } \\
\text { Total }\end{array}$} & & & & & & & & & \\
\hline & 27,197 & 5,915 & -- & 4,784 & -- & -- & 15,324 & -- & 1,174 \\
\hline & 502 & -- & -- & 103 & -- & -- & 178 & 30 & 191 \\
\hline & 27,699 & 5,915 & -- & 4,887 & -- & $=$ & 15,502 & 30 & 1,365 \\
\hline \multicolumn{10}{|l|}{ Hardwoods } \\
\hline White oak & 3,543 & -- & -- & -- & -- & 24 & 1,231 & 55 & 2,233 \\
\hline Red oak & 96 & -- & -- & -- & -- & 19 & 107 & -- & -30 \\
\hline Hickory & 28 & -- & -- & -- & -- & -- & 22 & -- & 6 \\
\hline Basswood & 1,015 & -- & -- & -- & -- & 35 & 980 & -- & -- \\
\hline Soft maple & 386 & -- & -- & -- & - & - & 386 & -- & -- \\
\hline Boxelder & 369 & -- & -- & -- & -- & -- & 369 & -- & -- \\
\hline Elm & 316 & -- & -- & -- & -- & -237 & 547 & -- & 6 \\
\hline Green ash & 1,095 & -- & -- & -- & 64 & -367 & 1,231 & 2 & 165 \\
\hline Cottonwood & 4,690 & -- & -- & 700 & -- & -- & 3,173 & 149 & 668 \\
\hline Black willow & 263 & -- & -- & -- & -- & -- & 449 & -239 & 53 \\
\hline Hackberry & 2,571 & -- & -- & -- & -- & -- & 2,516 & -- & 55 \\
\hline Elack walnut & 236 & -- & -- & -- & -- & - & 72 & -- & 164 \\
\hline Other hardwoods & 422 & -- & -- & -- & $=-$ & -- & 308 & 14 & 100 \\
\hline Tota 1 & 15,030 & -- & -- & 700 & 64 & -526 & 11,391 & -19 & 3,420 \\
\hline All species & 42,729 & 5,915 & -- & 5,587 & 64 & -526 & 26,893 & 11 & 4,785 \\
\hline
\end{tabular}

I/International $1 / 4$-inch rule.

Table 64.--Net annual growth of growing stock on commercjal forest land by species group and forest type, Nebraska, 1982 (In thousand cubic feet)

\begin{tabular}{|c|c|c|c|c|c|c|c|c|c|}
\hline \multirow[b]{2}{*}{ Species group } & \multirow[b]{2}{*}{$\begin{array}{l}\text { All } \\
\text { types }\end{array}$} & \multicolumn{8}{|c|}{ Forest type } \\
\hline & & $\begin{array}{l}\text { Ponderosa } \\
\text { pine }\end{array}$ & $\begin{array}{l}\text { Eastern } \\
\text { redcedar- } \\
\text { hardwood }\end{array}$ & $\begin{array}{l}\text { Oak- } \\
\text { hickory }\end{array}$ & $\begin{array}{l}\text { Bur } \\
\text { oak }\end{array}$ & $\begin{array}{l}\text { Elm-ast- } \\
\text { cottonwood }\end{array}$ & Cottonwood & $\begin{array}{l}\text { Lowland } \\
\text { plains } \\
\text { hardwoods }\end{array}$ & Nonstocked \\
\hline \\
\hline $\begin{array}{l}\text { Ponderosa pine } \\
\text { Eastern redcedar }\end{array}$ & $\begin{array}{r}5,415 \\
623\end{array}$ & $\begin{array}{r}5,336 \\
-\end{array}$ & $\begin{array}{r}5 \\
439 \\
\end{array}$ & -- & $\begin{array}{r}32 \\
5\end{array}$ & $\begin{array}{r}3 \\
98\end{array}$ & $\overline{42}$ & $\begin{array}{l}29 \\
39\end{array}$ & 10 \\
\hline Total & 6,038 & 5,336 & 444 & $=$ & 37 & 101 & 42 & 68 & 10 \\
\hline \multicolumn{10}{|l|}{ Hardwoods } \\
\hline White oak & 718 & 8 & 47 & 110 & 442 & 35 & -- & 76 & -- \\
\hline Red oak & 70 & -- & 4 & 41 & -- & 7 & -- & 18 & - \\
\hline Hickory & 125 & - & $\ldots$ & 74 & 13 & -- & -- & 38 & -- \\
\hline Basswood & 112 & -- & 6 & 35 & - & 1 & -- & 70 & -- \\
\hline Soft maple & 199 & - & - & -- & -- & 194 & -. & 5 & -- \\
\hline Boxelder & 180 & -- & -- & -- & -- & 138 & -- & 42 & -- \\
\hline Elm & 288 & -- & -90 & -40 & 1 & 344 & 32 & 41 & -- \\
\hline Green ash & 408 & -- & 18 & -58 & 44 & 254 & 53 & 97 & -- \\
\hline Cott onwood & 1,703 & -- & 49 & 8 & -237 & 543 & 1,321 & 7 & 12 \\
\hline Black willow & 137 & -- & 5 & -- & -- & 146 & -16 & 2 & -- \\
\hline Hackberry & 665 & -- & 2 & 23 & 41 & 175 & 7 & 417 & -- \\
\hline 81 ack walnut & 135 & -- & 1 & 28 & 3 & 25 & -- & 78 & -- \\
\hline Other hardwoods & 321 & -- & 14 & 7 & 5 & 139 & -- & 156 & - \\
\hline Total & 5,061 & 8 & 56 & 228 & 312 & 2,001 & 1,397 & 1,047 & 12 \\
\hline All species & 11,099 & 5,344 & 500 & 228 & 349 & 2,102 & 1,439 & 1,115 & 22 \\
\hline
\end{tabular}


Table 65.--Net annual growth of sawtimber on commercial forest land by species group and forest type, Nebraska, 1982 (In thousand board feet) 1 !

\begin{tabular}{|c|c|c|c|c|c|c|c|c|c|}
\hline \multirow[b]{2}{*}{ Species group } & \multirow[b]{2}{*}{$\begin{array}{c}\text { All } \\
\text { types }\end{array}$} & \multicolumn{8}{|c|}{ Forest type } \\
\hline & & $\begin{array}{c}\text { Ponderosa } \\
\text { pine }\end{array}$ & $\begin{array}{l}\text { Eastern } \\
\text { redcedar- } \\
\text { hardwood }\end{array}$ & $\begin{array}{c}\text { Oak- } \\
\text { hickory }\end{array}$ & $\begin{array}{l}\text { Bur } \\
\text { oak }\end{array}$ & $\begin{array}{c}\text { Elm-ash- } \\
\text { cottonwood }\end{array}$ & Cottonwood & $\begin{array}{l}\text { Lowland } \\
\text { plains } \\
\text { hardwoods }\end{array}$ & Nonstocked \\
\hline \multirow{3}{*}{$\begin{array}{l}\text { Softwoods } \\
\text { Ponderosa pine } \\
\text { Eastern redcedar } \\
\text { Total }\end{array}$} & & & & & & & & & \\
\hline & $\begin{array}{r}27,197 \\
502 \\
\end{array}$ & $\begin{array}{r}26,847 \\
-- \\
\end{array}$ & $\begin{array}{r}34 \\
292 \\
\end{array}$ & -- & $\begin{array}{l}74 \\
-- \\
\end{array}$ & $\begin{array}{l}23 \\
58 \\
\end{array}$ & 102 & $\begin{array}{r}178 \\
50 \\
\end{array}$ & 41 \\
\hline & 27,699 & 26,847 & 326 & -- & 74 & 81 & 102 & 228 & 41 \\
\hline \multicolumn{10}{|l|}{ Hardwoods } \\
\hline White oak & 3,543 & -- & 185 & 291 & 1,468 & 144 & -- & 1,455 & -- \\
\hline Red oak & 96 & -- & 7 & -11 & -- & 29 & -- & 71 & -- \\
\hline Hickory & 28 & -- & -- & 21 & 6 & 1 & -- & -- & -- \\
\hline Basswood & 1,015 & -- & 23 & 84 & -- & 3 & -- & 905 & -- \\
\hline Soft maple & 386 & -- & -- & -- & -- & 386 & -- & -- & -- \\
\hline Boxelder & 369 & -- & -- & -- & -- & 258 & $\cdots$ & 111 & -- \\
\hline $\mathrm{Elm}$ & 316 & -- & -467 & -237 & 6 & 136 & 812 & 66 & -- \\
\hline Green ash & 1,095 & -- & 67 & -332 & 99 & 809 & 155 & 297 & -- \\
\hline Cottonwood & 4,690 & -- & 190 & 16 & $-1,067$ & 1,613 & 3,895 & 18 & 25 \\
\hline Black willow & 263 & -- & -- & -- & -- & 353 & -95 & 5 & -- \\
\hline Hackberry & 2,571 & -- & -- & $=$ & 64 & 194 & 34 & 2,279 & -- \\
\hline Black walnut & 236 & -- & 2 & 86 & 15 & 11 & -- & 122 & -- \\
\hline other hardwoods & 422 & -- & - & 33 & 14 & 51 & $=$ & 324 & -- \\
\hline Total & 15,030 & - & 7 & -49 & 605 & 3,988 & 4,801 & 5,653 & 25 \\
\hline All species & 42,729 & 26,847 & 333 & -49 & 679 & 4,069 & 4,903 & 5,881 & 66 \\
\hline
\end{tabular}

1/ International $1 / 4$-inch rule. 

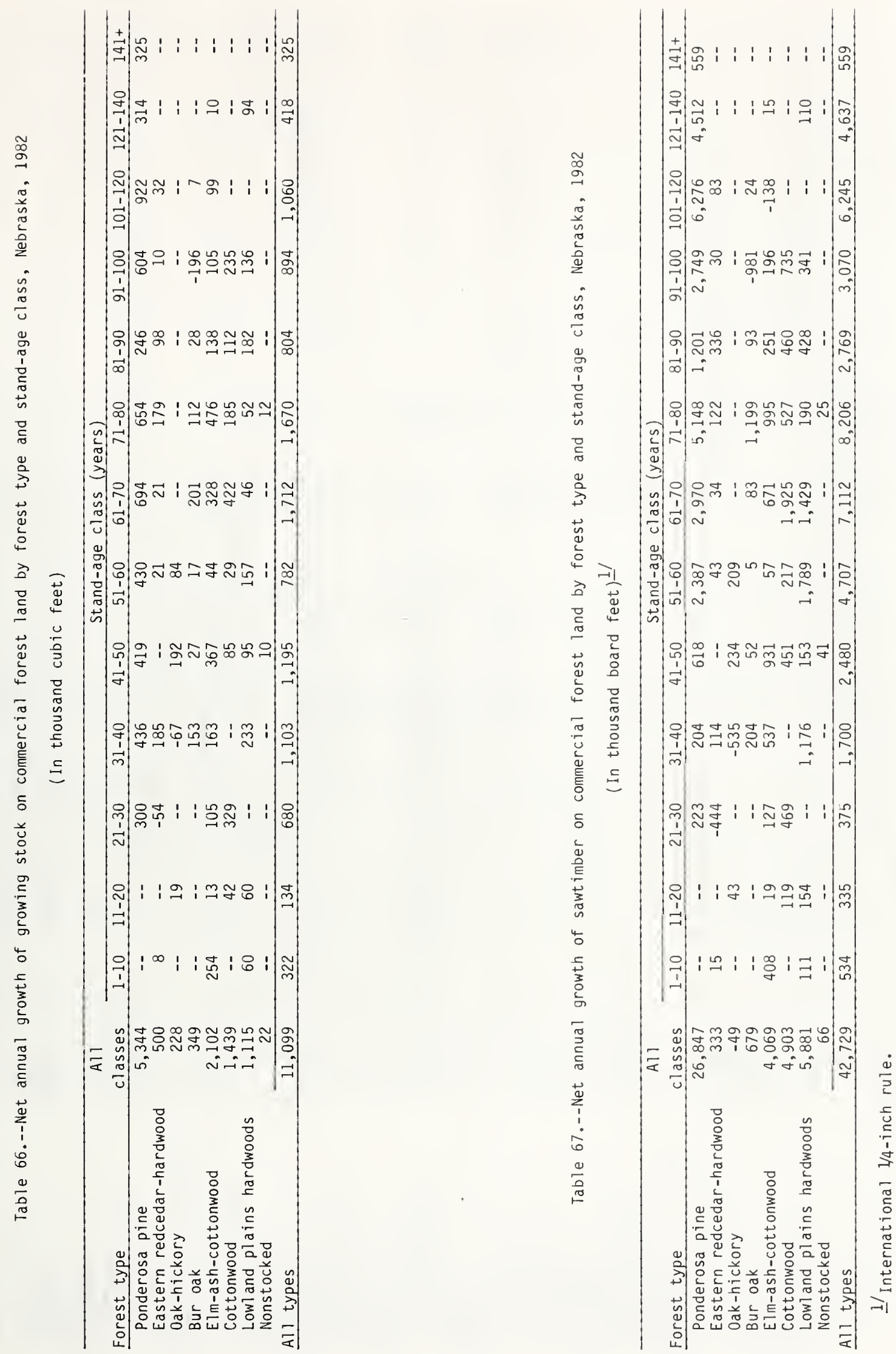


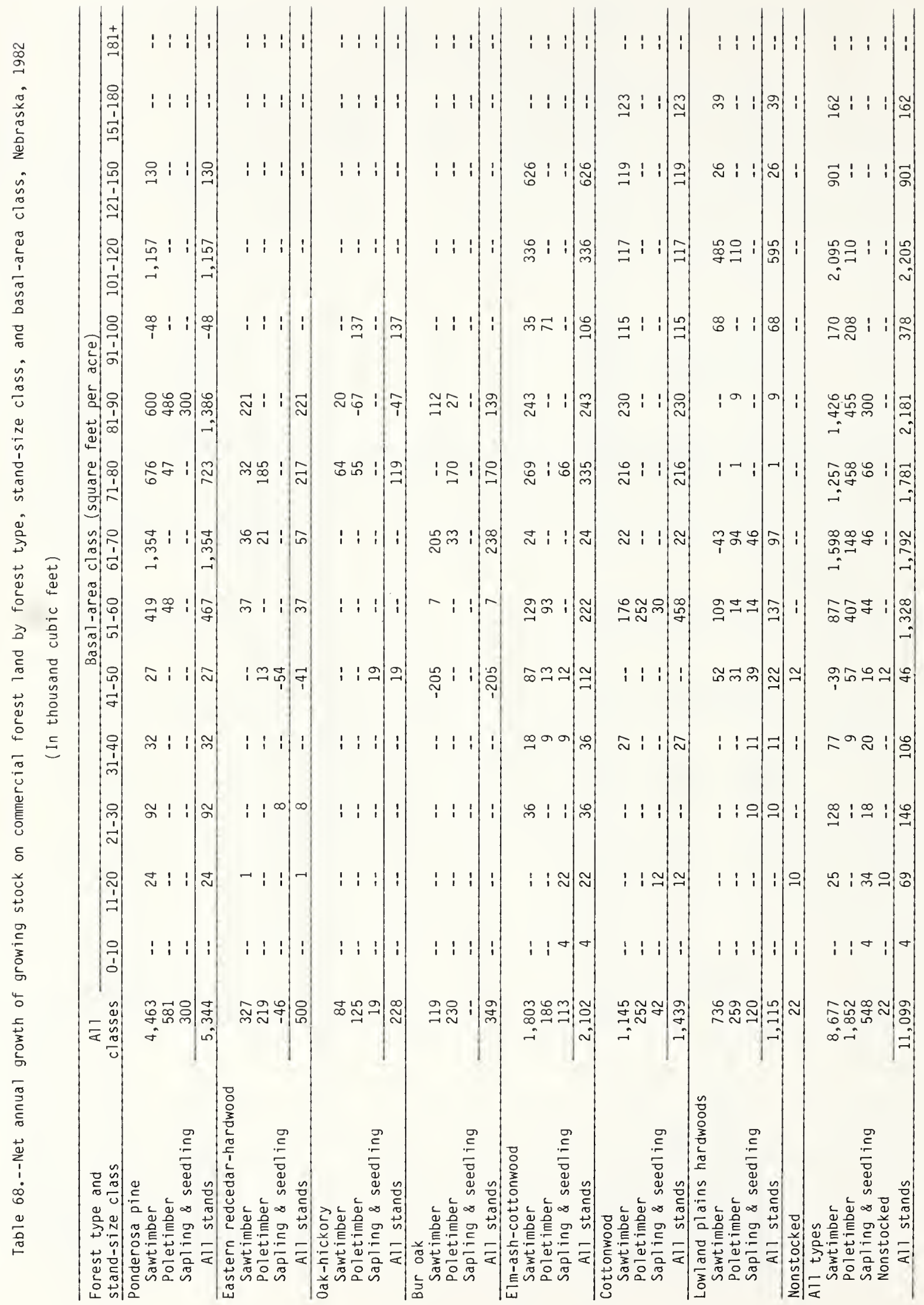




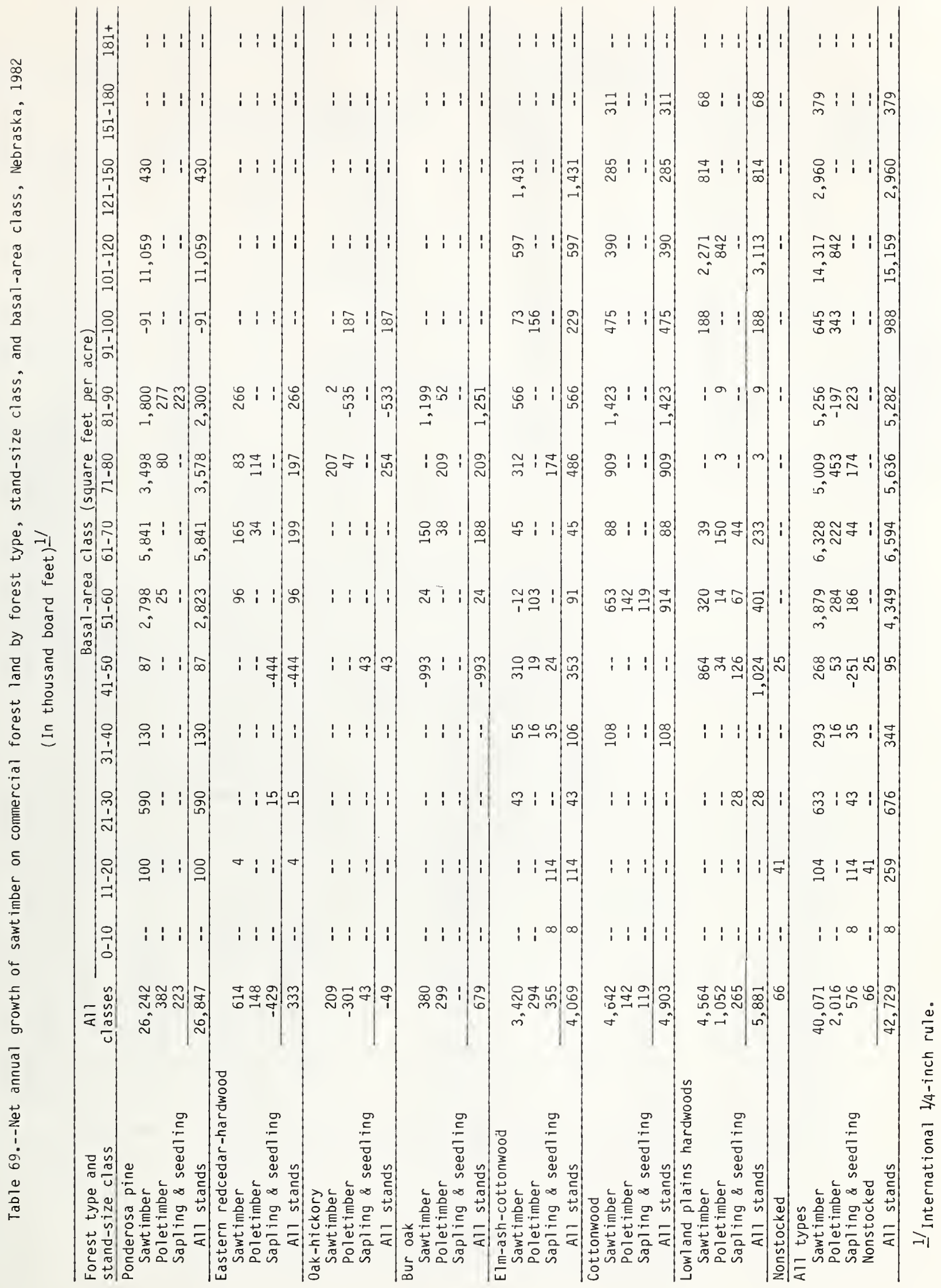


Table 70.--Timber removals from growing stock on commercial forest land, by species group and Forest Survey Unit, Nebraska, 1982

(In thousand cubic feet)

\begin{tabular}{|c|c|c|c|}
\hline \multirow[b]{2}{*}{ Species group } & \multirow[b]{2}{*}{$\begin{array}{c}\text { All } \\
\text { Units }\end{array}$} & \multicolumn{2}{|c|}{ Forest Survey Unit } \\
\hline & & $\begin{array}{c}\text { Eastern } \\
\text { Unit }\end{array}$ & $\begin{array}{c}\text { Western } \\
\text { Unit }\end{array}$ \\
\hline $\begin{array}{l}\text { Softwoods } \\
\text { Ponderosa pine } \\
\text { Eastern redcedar }\end{array}$ & $\begin{array}{r}517 \\
56 \\
\end{array}$ & 34 & $\begin{array}{r}517 \\
22 \\
\end{array}$ \\
\hline Total & 573 & 34 & 539 \\
\hline $\begin{array}{l}\text { Hardwoods } \\
\text { White oak } \\
\text { Red oak } \\
\text { Hickory } \\
\text { Basswood } \\
\text { Soft maple } \\
\text { Boxelder } \\
\text { Elm } \\
\text { Green ash } \\
\text { Cottonwood } \\
\text { Black willow } \\
\text { Hackberry } \\
\text { Black walnut } \\
\text { Other hardwoods }\end{array}$ & $\begin{array}{r}2,233 \\
247 \\
165 \\
30 \\
27 \\
319 \\
281 \\
790 \\
3,190 \\
401 \\
449 \\
166 \\
479\end{array}$ & $\begin{array}{r}1,659 \\
247 \\
165 \\
28 \\
27 \\
301 \\
262 \\
574 \\
2,904 \\
305 \\
423 \\
162 \\
363\end{array}$ & $\begin{array}{r}574 \\
-- \\
-- \\
2 \\
-- \\
18 \\
19 \\
216 \\
286 \\
96 \\
26 \\
4 \\
116\end{array}$ \\
\hline Total & 8,777 & 7,420 & 1,357 \\
\hline All species & 9,350 & 7,454 & 1,896 \\
\hline
\end{tabular}

Table 71.--Timber removals from sawtimber on commercial forest land, by species yroup and Forest Survey Unit, Nebraska, 1982

(In thousand board feet) 1 /

\begin{tabular}{|c|c|c|c|}
\hline & & Forest & vey Unit \\
\hline Species group & $\begin{array}{l}\text { All } \\
\text { Units }\end{array}$ & $\begin{array}{c}\text { Eastern } \\
\text { Unit } \\
\end{array}$ & $\begin{array}{c}\text { Western } \\
\text { Unit } \\
\end{array}$ \\
\hline Softwoods & & & \\
\hline $\begin{array}{l}\text { Ponderosa pine } \\
\text { Eastern redcedar }\end{array}$ & $\begin{array}{r}2,376 \\
170 \\
\end{array}$ & 139 & $\begin{array}{r}2,376 \\
31 \\
\end{array}$ \\
\hline Total & 2,546 & 139 & 2,407 \\
\hline Hardwoods & & & \\
\hline White oak & 6,190 & 4,618 & 1,572 \\
\hline Red oak & 674 & 674 & - \\
\hline Hickory & 435 & 435 & -- \\
\hline Basswood & 93 & 89 & 4 \\
\hline Soft maple & 101 & 101 & -- \\
\hline Boxelder & 1,269 & 1,269 & -- \\
\hline Elm & 789 & 730 & 59 \\
\hline Green ash & 2,161 & 1,576 & 585 \\
\hline Cottonwood & 17,356 & 15,962 & 1,394 \\
\hline Black willow & 1,084 & 823 & 261 \\
\hline Hackberry & 1,217 & 1,145 & 72 \\
\hline Black walnut & 1,044 & 1,016 & 28 \\
\hline Other hardwoods & 1,305 & $\begin{array}{r}989 \\
\end{array}$ & 316 \\
\hline Total & 33,718 & 29,427 & 4,291 \\
\hline All species & 36,264 & 29,566 & 6,698 \\
\hline
\end{tabular}

1/ International 1/4-inch rule.

Table 72.--Timber removals from growing stock and sawtimber on commercial forest land by species group, Nebraska, 1953 and 1982

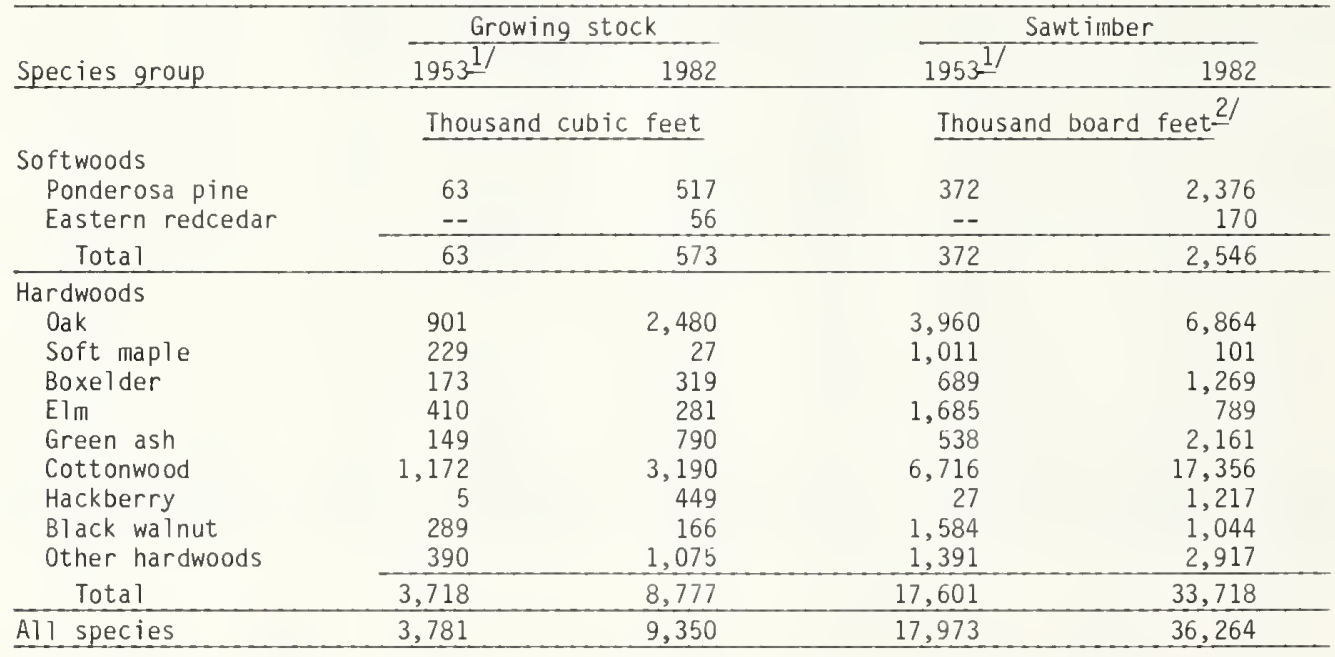

I/ Figures have been adjusted from those published after the 1953 survey to conform to 1982 volumes because of changes in survey definitions and procedures.

2/ International $1 / 4$-inch rule. 
Table 73.--Timber removals from growing stock and sawtimber on commercial forest land by item and species category, Nebraska, 1982

GROWING STOCK

\begin{tabular}{|c|c|c|c|c|c|c|c|c|}
\hline \multirow[b]{2}{*}{ Item } & \multirow[b]{2}{*}{$\begin{array}{c}\text { All } \\
\text { species }\end{array}$} & \multicolumn{7}{|c|}{ Species category } \\
\hline & & Softwoods & Oak & $\begin{array}{c}\text { Elm- } \\
\text { hackberry }\end{array}$ & Ash & Cottonwood & Walnut & $\begin{array}{c}\text { Other } \\
\text { hardwoods }\end{array}$ \\
\hline ROUNDWOUD PRODUCTS & --- & ---- & --- & -Thousand & cubic fe & ; - - - & --- & ---- \\
\hline Saw logs & 2,915 & 313 & 55 & 5 & 8 & 2,377 & 140 & 17 \\
\hline Veneer logs & 112 & -- & -- & -- & -- & 99 & 13 & -- \\
\hline Cooperage logs & 18 & -- & 18 & -- & -- & -- & -- & -- \\
\hline Fuelwood & 5,167 & 9 & 2,327 & 683 & 756 & 248 & -- & 1,144 \\
\hline Posts & 79 & 60 & 11 & 1 & -- & 1 & -- & 6 \\
\hline Pulpwood & 12 & 12 & -- & -- & -- & -- & -- & -- \\
\hline other & 94 & 94 & -- & -- & -- & -- & -- & -- \\
\hline Total & 8,397 & 488 & 2,411 & 689 & 764 & 2,725 & 153 & 1,167 \\
\hline LOGGING RES IDUE & 440 & 39 & 20 & -- & 3 & 364 & 13 & 1 \\
\hline OTHER REMOVALS & 513 & 46 & 49 & 41 & 23 & 101 & -- & 253 \\
\hline ALL TIMBER REMUVALS & 9,350 & 573 & 2,480 & 730 & 790 & 3,190 & 166 & 1,421 \\
\hline \multicolumn{9}{|c|}{ SAWT IMBER } \\
\hline ROUNDWOOD PRODUCTS & --- & ---- & --- & -Thousand & board & $1 /-\ldots$ & --- & --- \\
\hline Saw logs & 18,551 & 1,767 & 265 & 42 & 51 & 15,420 & 911 & 95 \\
\hline Veneer logs & 518 & -- & -- & -- & -- & 433 & 85 & -- \\
\hline Cooperage logs & 106 & -- & 106 & -- & -- & -- & -- & -- \\
\hline Fuelwood & 14,190 & 27 & 6,415 & 1,886 & 2,081 & 682 & -- & 3,099 \\
\hline Posts & 93 & 37 & 30 & 3 & -- & 3 & -- & 20 \\
\hline Pulpwood & 18 & 18 & -- & -- & -- & -- & -- & -- \\
\hline other & 512 & 512 & - & $=-$ & $=-$ & -- & $=$ & -- \\
\hline Total & 33,988 & 2,361 & 6,816 & 1,931 & 2,132 & 16,538 & 996 & 3,214 \\
\hline LOGGING RESIDUE & 905 & 77 & 32 & -- & 7 & 739 & 48 & 2 \\
\hline OTHER REMOVALS & 1,371 & 108 & 16 & 75 & 22 & 79 & -- & 1,071 \\
\hline ALL TIMBER REMOVALS & 36,264 & 2,546 & 6,864 & 2,006 & 2,161 & 17,356 & 1,044 & 4,287 \\
\hline
\end{tabular}

\section{1/International $1 / 4$-inch rule.}

Table 74.--Net annual growth and removals of growing stock on commercial forest land by species group, Nebraska, 1982

(In thousand cubic feet)

\begin{tabular}{|c|c|c|}
\hline Species group & $\begin{array}{l}\text { Net annual } \\
\text { growth }\end{array}$ & $\begin{array}{c}\text { Annual timber } \\
\text { removals }\end{array}$ \\
\hline $\begin{array}{l}\text { Softwoods } \\
\text { Ponderosa pine } \\
\text { Eastern redcedar }\end{array}$ & $\begin{array}{r}5,415 \\
623 \\
\end{array}$ & $\begin{array}{r}517 \\
56 \\
\end{array}$ \\
\hline Total & 6,038 & 573 \\
\hline $\begin{array}{l}\text { Hardwoods } \\
\text { White oak } \\
\text { Red oak } \\
\text { Hickory } \\
\text { Basswood } \\
\text { Soft maple } \\
\text { Boxelder } \\
\text { Elm } \\
\text { Green ash } \\
\text { Cottonwood } \\
\text { Black willow } \\
\text { Hackberry } \\
\text { Black walnut } \\
\text { Other hardwoods }\end{array}$ & $\begin{array}{r}718 \\
70 \\
125 \\
112 \\
199 \\
180 \\
288 \\
408 \\
1,703 \\
137 \\
665 \\
135 \\
321 \\
\end{array}$ & $\begin{array}{r}2,233 \\
247 \\
165 \\
30 \\
27 \\
319 \\
281 \\
790 \\
3,190 \\
401 \\
449 \\
166 \\
479 \\
\end{array}$ \\
\hline Total & 5,061 & 8,777 \\
\hline A11 species & 11,099 & 9,350 \\
\hline
\end{tabular}


Table 75.--Net annual growth and removals of sawtimber on commercial forest land by species group, Nebraska, 1982

(In thousand board feet) $1 /$

\begin{tabular}{lrr}
\hline Species group & $\begin{array}{r}\text { Net annual } \\
\text { growth }\end{array}$ & $\begin{array}{c}\text { Annual timber } \\
\text { removals }\end{array}$ \\
\hline Softwoods & 27,197 & \\
Ponderosa pine & 502 & 2,376 \\
Eastern redcedar & 27,699 & 170 \\
$\quad$ Total & & 2,546 \\
\hline Hardwoods & 3,543 & 6,190 \\
White oak & 96 & 674 \\
Red oak & 28 & 435 \\
Hickory & 1,015 & 93 \\
Basswood & 386 & 101 \\
Soft maple & 369 & 1,269 \\
Boxelder & 316 & 789 \\
Elm & 1,095 & 2,161 \\
Green ash & 4,690 & 17,356 \\
Cottonwood & 263 & 1,084 \\
Black willow & 2,571 & 1,217 \\
Hackberry & 236 & 1,044 \\
Black walnut & 422 & 1,305 \\
Other hardwoods & 15,030 & 33,718 \\
\hline$\quad$ Total & 42,729 & 36,264 \\
\hline All species & & \\
\hline
\end{tabular}

1/ International 1/4-inch rule.

Table 76.--Net annual growth and removals of growing stock on commercial forest land by ownership class and softwoods and hardwoods, Nebraska, 1982

(In thousand cubic feet)

\begin{tabular}{|c|c|c|c|c|c|c|}
\hline \multirow[b]{2}{*}{ Ownership class } & \multicolumn{3}{|c|}{ Net annual growth } & \multicolumn{3}{|c|}{ Annual timber removals } \\
\hline & $\begin{array}{c}\text { All } \\
\text { species }\end{array}$ & Softwoods & Hardwoods & $\begin{array}{c}\text { All } \\
\text { species }\end{array}$ & Sof twoods & Hardwoods \\
\hline \multicolumn{7}{|l|}{ PUBLIC } \\
\hline National Forest & 1,479 & 1,479 & -- & 10 & 10 & -- \\
\hline State & 538 & 380 & 158 & 16 & -- & 16 \\
\hline County \& municipal & 21 & -- & 21 & -- & -- & -- \\
\hline Indian & -46 & -- & -46 & 58 & -- & 58 \\
\hline Total & 1,992 & 1,859 & 133 & 84 & 10 & 74 \\
\hline \multirow{2}{*}{\multicolumn{7}{|c|}{$\begin{array}{l}\text { PRIVATE } \\
\text { Farmer and }\end{array}$}} \\
\hline & & & & & & \\
\hline All owners & 11099 & 6.038 & 5061 & 9.350 & 573 & 8.777 \\
\hline All owllets & 11,099 & 0,030 & 3,001 & 9,350 & $3 / 2$ & 0,111 \\
\hline
\end{tabular}

Table 77.--Net annual growth and removals of sawtimber on commercial forest land by ownership class and softwoods and hardwoods, Nebraska, 1982

(In thousand board feet) 1 /

\begin{tabular}{|c|c|c|c|c|c|c|}
\hline \multirow[b]{2}{*}{ Ownership class } & \multicolumn{3}{|c|}{ Net annual growth } & \multicolumn{3}{|c|}{ Annual timber removals } \\
\hline & $\begin{array}{c}\text { All } \\
\text { species }\end{array}$ & Softwoods & Hardwoods & $\begin{array}{c}\text { All } \\
\text { species }\end{array}$ & Softwoods & Hardwoods \\
\hline $\begin{array}{l}\text { PUBLIC } \\
\text { National Forest } \\
\text { State } \\
\text { County \& municipal } \\
\text { Indian }\end{array}$ & $\begin{array}{r}5,915 \\
5,587 \\
64 \\
-526 \\
\end{array}$ & $\begin{array}{r}5,915 \\
4,887 \\
-- \\
-- \\
\end{array}$ & $\begin{array}{r}-- \\
700 \\
64 \\
-526 \\
\end{array}$ & $\begin{array}{r}23 \\
22 \\
-- \\
291 \\
\end{array}$ & $\begin{array}{l}23 \\
-- \\
-- \\
-- \\
\end{array}$ & $\begin{array}{r}-- \\
22 \\
-- \\
291 \\
\end{array}$ \\
\hline Total & 11,040 & 10,802 & 238 & 336 & 23 & 313 \\
\hline $\begin{array}{l}\text { PRIVATE } \\
\text { Farmer and } \\
\text { Misc. private }\end{array}$ & 31,689 & 16,897 & 14,792 & 35,928 & 2,523 & 33,405 \\
\hline All owners & 42,729 & 27,699 & 15,030 & 36,264 & 2,546 & 33,718 \\
\hline
\end{tabular}

1/ International 1/4-inch rule. 
Table 78.--Net annual mortality of growing stock on commercial forest land by softwoods and hardwoods, Nebraska, 1955 and 1982

(In thousand cubic feet)

\begin{tabular}{lrr}
\hline Species & $19551 /$ & 1982 \\
\hline Softwoods & 200 & 438 \\
Hardwoods & 1,700 & 1,745 \\
All species & 1,900 & 2,183 \\
\hline
\end{tabular}

1/Figures have been adjusted from those published after the 1955 survey to conform to 1982 volumes because of changes in survey definitons and procedures.

Table 79.--Annual mortality of growing stock on commercial forest land by species group and cause, Nebraska, 1982

(In thousand cubic feet)

\begin{tabular}{|c|c|c|c|c|c|c|c|c|}
\hline \multirow[b]{2}{*}{ Species group } & \multirow[b]{2}{*}{$\begin{array}{c}\text { All } \\
\text { Causes }\end{array}$} & \multicolumn{7}{|c|}{ Cause } \\
\hline & & Insects & Disease & Fire & Animals & Weather & Suppression & $\begin{array}{l}\text { Unknown } \\
\text { and other }\end{array}$ \\
\hline \multicolumn{9}{|l|}{ Softwoods } \\
\hline $\begin{array}{l}\text { Ponderosa pine } \\
\text { Eastern redcedar }\end{array}$ & $\begin{array}{r}401 \\
37\end{array}$ & $\begin{array}{l}72 \\
--\end{array}$ & $\begin{array}{l}84 \\
--\end{array}$ & -- & -- & -- & -- & $\begin{array}{r}245 \\
37\end{array}$ \\
\hline Total & 438 & 72 & 84 & -- & -- & -- & -- & 282 \\
\hline \multicolumn{9}{|l|}{ Hardwoods } \\
\hline White oak & 77 & -- & -- & -- & - & -- & -- & 77 \\
\hline Red oak & 72 & -- & -- & - & -- & 72 & -- & -- \\
\hline Hickory & -- & -- & -- & -- & -- & -- & -- & -- \\
\hline Basswood & -- & -- & -- & -- & -- & -- & $\ldots$ & $\ldots$ \\
\hline Soft maple & - & -- & -- & - & -- & - & -- & -- \\
\hline Boxelder & -- & -- & -- & -- & -- & -- & -- & -- \\
\hline Elm & 311 & -- & 136 & - & -- & -- & -- & 175 \\
\hline Green ash & 106 & -- & -- & -. & -- & -- & -- & 106 \\
\hline Cottonwood & 898 & -- & -- & -- & -- & 225 & - & 673 \\
\hline Black willow & 208 & -- & 96 & -. & -- & - & -- & 112 \\
\hline Hackberry & -- & -- & .. & - & -- & -- & -. & -- \\
\hline Black walnut & -. & -- & -- & -- & -- & -- & - & - \\
\hline Other hardwoods & 73 & -- & -- & -- & -- & -- & $=$ & 73 \\
\hline Total & 1,745 & -- & 232 & $\ldots$ & - & 297 & -- & 1,216 \\
\hline All species & 2,183 & 72 & 316 & -- & -- & 297 & -- & 1,498 \\
\hline
\end{tabular}


Table 80.--Annual mortality of sawtimber on commercial forest 1 and by species group and cause, Nebraska, 1982

(In thousand board feet) 1 /

\begin{tabular}{|c|c|c|c|c|c|c|c|c|}
\hline \multirow[b]{2}{*}{ Species group } & \multirow[b]{2}{*}{$\begin{array}{c}\text { Al1 } 1 \\
\text { causes }\end{array}$} & \multicolumn{7}{|c|}{ Cause } \\
\hline & & Insects & Disease & Fire & Animals & Weather & Suppression & $\begin{array}{l}\text { Unknown } \\
\text { and other }\end{array}$ \\
\hline \multirow{4}{*}{$\begin{array}{l}\text { Softwoods } \\
\text { Ponderosa pine } \\
\text { Eastern redcedar } \\
\text { Total }\end{array}$} & & & & & & & & \\
\hline & 1,566 & 403 & -- & -- & -- & -- & $\cdots$ & 1,163 \\
\hline & -- & -- & -- & -- & -- & -- & -- & $\ldots$ \\
\hline & 1,566 & 403 & -- & -- & -- & -- & -- & 1,163 \\
\hline \multicolumn{9}{|l|}{ Hardwoods } \\
\hline White oak & -- & -- & -- & -- & -- & -- & -- & -- \\
\hline Red oak & 369 & -- & -- & -- & -- & 369 & -- & -- \\
\hline Hickory & -- & -- & -- & -- & -- & -- & -- & -- \\
\hline Basswood & -- & -- & -- & -- & -- & -- & -- & -- \\
\hline Soft maple & -- & -- & -- & -- & -- & -- & -- & -- \\
\hline Boxelder & -- & - & $\rightarrow$ & $\rightarrow$ & $\omega$ & -- & -- & -- \\
\hline Elm & 923 & -- & 467 & -- & -- & -- & -- & 456 \\
\hline Green ash & 367 & -- & -- & -- & -- & -- & -- & 367 \\
\hline Cottonwood & 3,502 & -- & -- & -- & -- & 835 & -- & 2,667 \\
\hline Black willow & 564 & -- & -- & -- & - & -- & -- & 564 \\
\hline Hackberry & -- & $-\infty$ & -- & -- & -- & -- & -- & -- \\
\hline Black walnut & -- & -- & -- & -- & -- & -- & -- & -- \\
\hline Other hardwoods & -- & -- & -- & $=$ & $\because$ & $\because-$ & -- & -- \\
\hline Total & 5,725 & -- & 467 & $=$ & $=$ & 1,204 & -- & 4,054 \\
\hline Al1 species & 7,291 & 403 & 467 & -- & -- & 1,204 & -- & 5,217 \\
\hline
\end{tabular}

1 International $1 / 4$-inch rule.

Table 81.--Annual mortality of growing stock and sawtimber on commercial forest land by ownership class and softwoods and hardwoods, Nebraska, 1982

\begin{tabular}{|c|c|c|c|c|c|c|}
\hline \multirow[b]{2}{*}{ Ownership class } & \multicolumn{3}{|c|}{ Growing stock } & \multicolumn{3}{|c|}{ Sawtimber } \\
\hline & $\begin{array}{c}\text { All } \\
\text { species }\end{array}$ & Softwoods & Hardwoods & $\begin{array}{c}\text { All } \\
\text { species }\end{array}$ & Softwoods & Hardwoods \\
\hline & - & usand cubic & et- - - - & $---I$ & sand board & $t \underline{1 /}-\cdots$ \\
\hline National Forest & 121 & 121 & -- & 385 & 385 & - \\
\hline State & 37 & 37 & -- & -- & -- & - \\
\hline County and municipal & -- & -- & -- & -- & -- & -- \\
\hline Ind $i$ an & 134 & -- & 134 & 620 & -- & 620 \\
\hline Farmer & 1,623 & 280 & 1,343 & 5,635 & 1,181 & 4,454 \\
\hline Misc. private corporation & 98 & -- & 98 & 282 & - & 282 \\
\hline Misc. private individual & 170 & -- & 170 & 369 & -- & 369 \\
\hline All owners & 2,183 & 438 & 1,745 & 7,291 & 1,566 & 5,725 \\
\hline
\end{tabular}

1/International $1 / 4$-inch rule. 


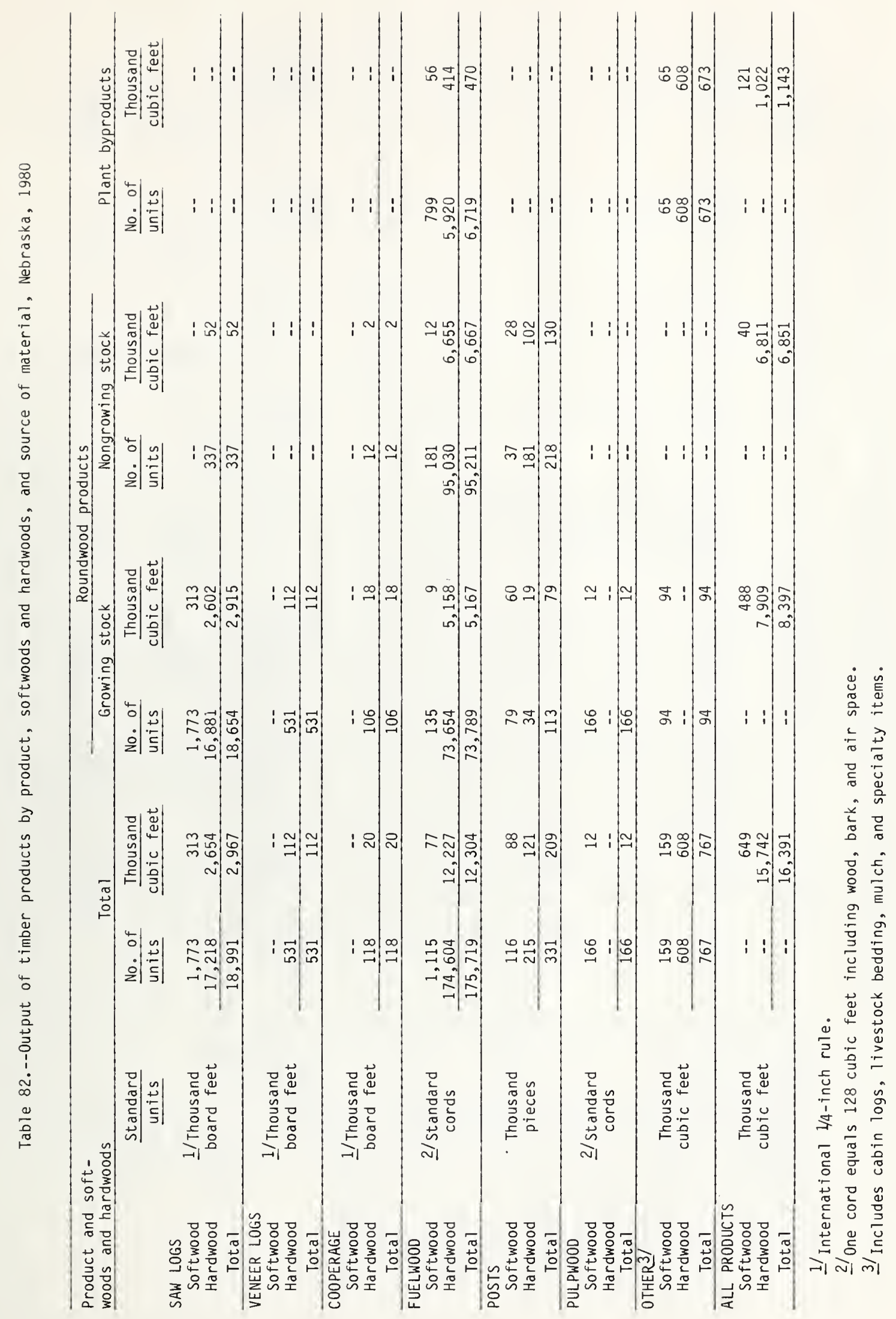




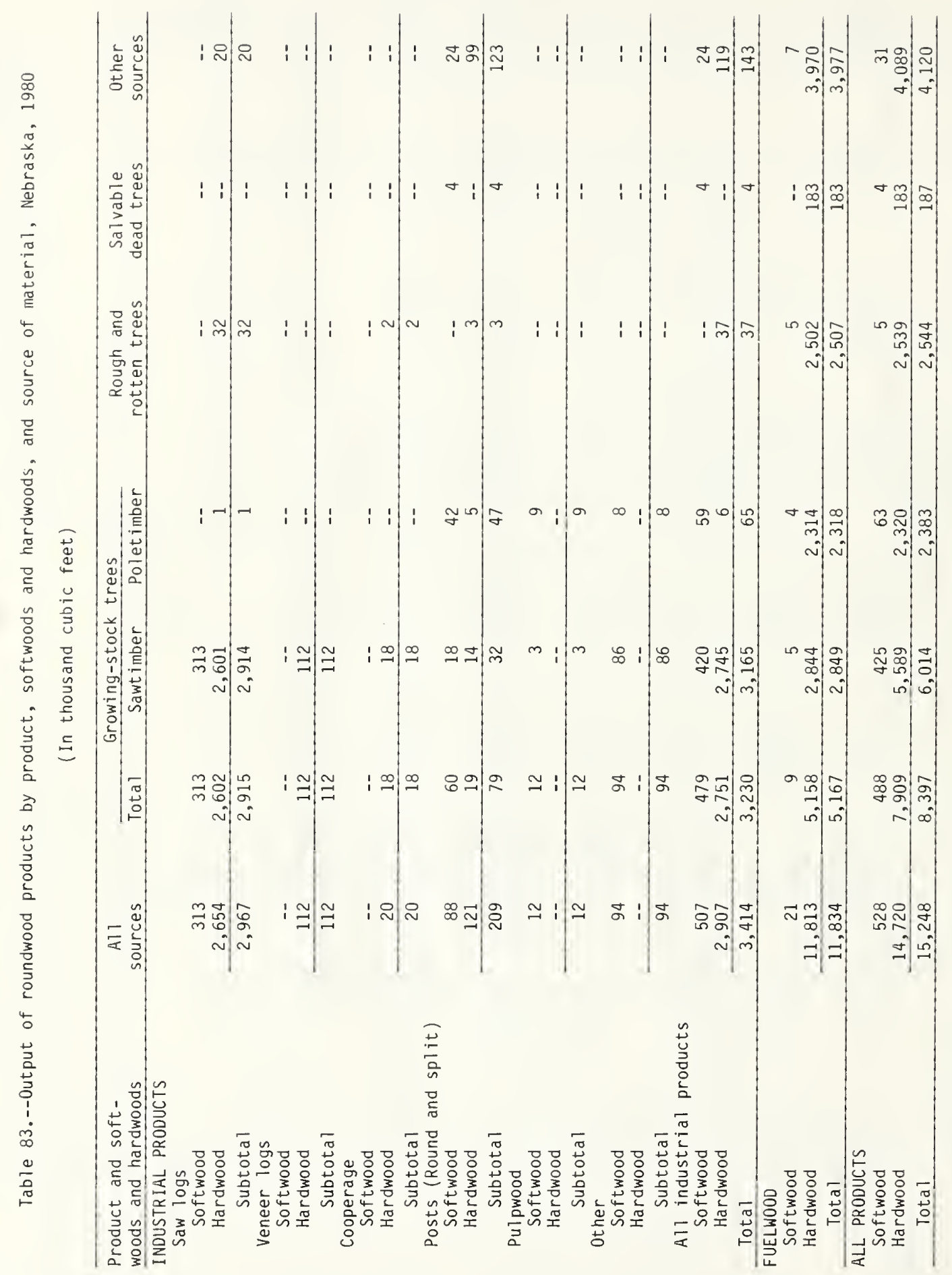




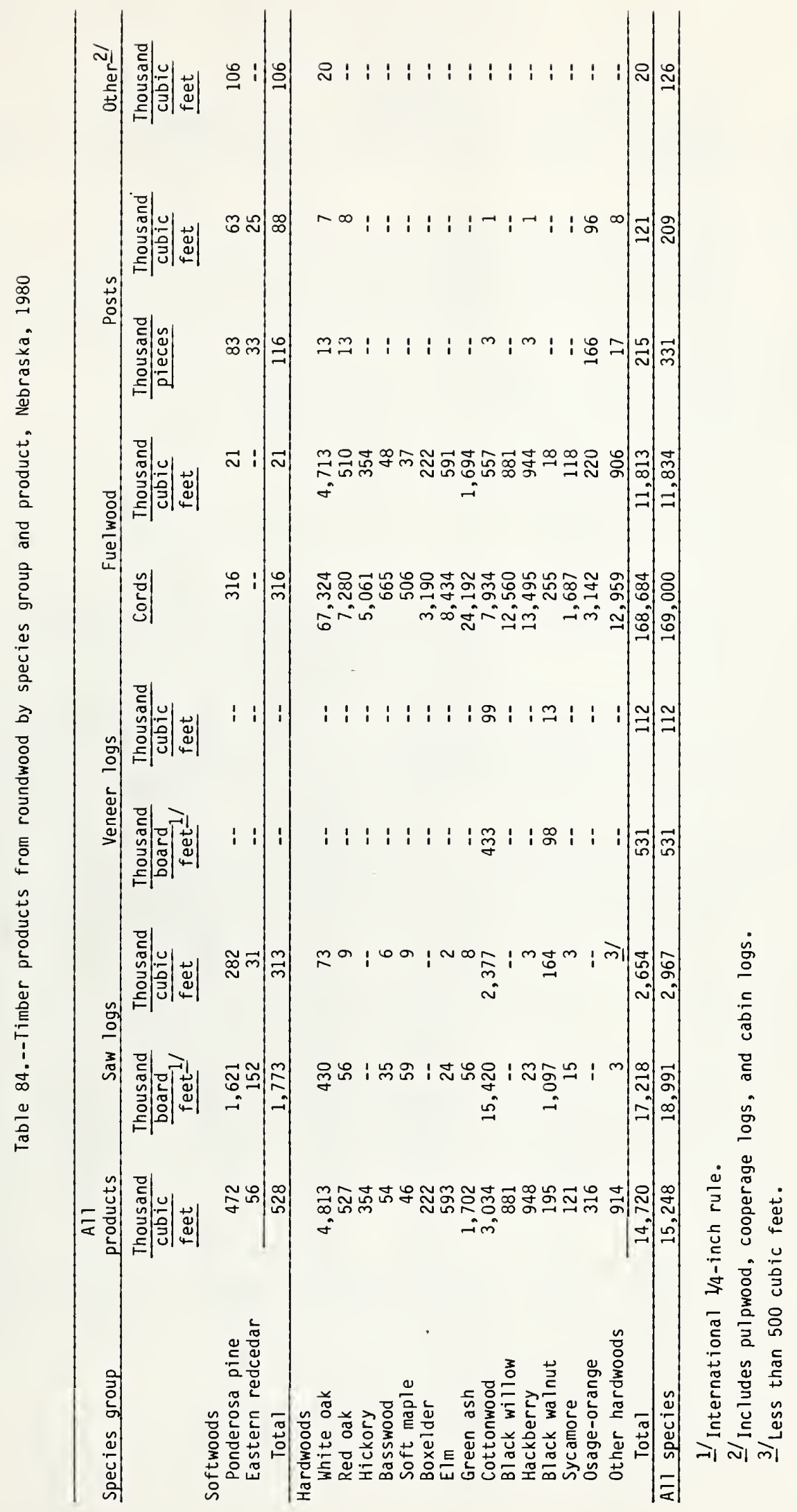


Table 85.--Volume of primary plant residue by type of use and kind of material, Nebraska, 1980

(In thousand cubic feet)

\begin{tabular}{|c|c|c|c|c|c|c|c|c|}
\hline \multirow[b]{3}{*}{ Type of use } & \multicolumn{6}{|c|}{ Kind of wood residue } & \multirow{2}{*}{\multicolumn{2}{|c|}{ Bark }} \\
\hline & \multicolumn{2}{|c|}{ Total } & \multicolumn{2}{|c|}{ Coarse $1 /$} & \multicolumn{2}{|c|}{ Fine $^{2 /}$} & & \\
\hline & Softwood & Hardwood & Softwood & Hardwood & Softwood & Hardwood & Softwood & Hardwood \\
\hline Fiber products & -- & -- & -- & -- & -- & -- & -- & - \\
\hline Industrial fuel & -- & -- & -- & -- & -- & -- & -- & -- \\
\hline Domestic fuel & 55.9 & 414.4 & 55.9 & 412.5 & -- & 1.9 & 34.8 & 312.2 \\
\hline Miscellaneous 3 / & 65.1 & 607.9 & 22.9 & 160.5 & 42.2 & 447.4 & 17.0 & 34.1 \\
\hline Not used4/ & 13.5 & 490.5 & 6.2 & 367.6 & 7.3 & 122.9 & 19.4 & 269.5 \\
\hline A11 uses & 134.5 & $1,512.8$ & 85.0 & 940.6 & 49.5 & 572.2 & 71.2 & 615.8 \\
\hline
\end{tabular}

1/Suitable for chipping such as slabs, edgings, veneer cores, etc.

2/ Not suitable for chipping such as sawdust, veneer clippings, etc.

3/Livestock bedding, mulch, small dimension, charcoal, and specialty items.

4/ Includes residue burned as waste.

Table 86.--A11 live tree biomass yields on commercial forest land by species group and forest type, Nebraska, 1983

(In pounds per acre green weight)

\begin{tabular}{|c|c|c|c|c|c|c|c|c|}
\hline \multirow[b]{2}{*}{ Species group } & \multicolumn{8}{|c|}{ Forest type } \\
\hline & $\begin{array}{c}\text { Ponderosa } \\
\text { pine }\end{array}$ & $\begin{array}{l}\text { Eastern } \\
\text { redcedar- } \\
\text { hardwood }\end{array}$ & $\begin{array}{c}\text { Oak- } \\
\text { hickory }\end{array}$ & $\begin{array}{l}\text { Bur } \\
\text { oak }\end{array}$ & $\begin{array}{c}\text { Elm-ash- } \\
\text { cottonwood }\end{array}$ & Cottonwood & $\begin{array}{l}\text { Lowland } \\
\text { plains } \\
\text { hardwoods }\end{array}$ & Nonstocked \\
\hline \multirow{5}{*}{$\begin{array}{l}\text { Softwoods } \\
\text { Ponderosa pine } \\
\text { Eastern redcedar } \\
\text { Rocky mountain juniper } \\
\text { Total }\end{array}$} & & & & & & & & \\
\hline & 100,200 & 1,345 & -- & 3,098 & 301 & -- & 1,337 & 6,033 \\
\hline & 389 & 20,927 & -- & 1,803 & 1,275 & 2,425 & 1,819 & -- \\
\hline & -- & $\ldots$ & -- & - & $=-$ & 783 & -- & - \\
\hline & 100,589 & 22,272 & -- & 4,901 & 1,576 & 3,208 & 3,156 & 6,033 \\
\hline \multicolumn{9}{|l|}{ Hardwoods } \\
\hline Select white oak & 6,016 & 13,346 & 39,395 & 91,863 & 3,205 & -- & 25,031 & -- \\
\hline Post oak & -- & -. & -- & -- & 109 & - & -- & - \\
\hline Northern red oak & -- & 1,060 & 16,933 & -- & 313 & -- & 1,053 & -- \\
\hline Black oak & -- & - - & 7,236 & -- & -- & -- & - & -- \\
\hline Shagbark hickory & -- & -- & 17,555 & -- & 150 & -- & 718 & - \\
\hline Bitternut hickory & -- & -- & 5,049 & 2,066 & -- & - & 564 & -- \\
\hline Basswood & -- & 1,513 & 6,986 & - & 83 & -- & 6,384 & - \\
\hline Soft maple & -- & -- & - & -- & 6,530 & -- & 369 & -- \\
\hline Boxelder & 469 & 2,241 & 1,236 & 1,882 & 11,278 & 429 & 6,129 & -- \\
\hline $\mathrm{E} / \mathrm{m}$ & -- & 2,048 & 10,808 & 340 & 12,825 & 2,093 & 7,931 & -. \\
\hline Green ash & 599 & 5,514 & 5,462 & 5,536 & 16,849 & 4,609 & 7,441 & -- \\
\hline Cottonwood & -- & 12,523 & 1,785 & 294 & 29,598 & 122,692 & 3,768 & 26,408 \\
\hline Black willow & -- & 704 & -- & -- & 6,734 & 6,565 & 316 & - \\
\hline Hackberry & -- & 1,535 & 2,676 & 3,713 & 2,161 & 513 & 22,515 & -- \\
\hline Black walnut & -- & 321 & 5,270 & 329 & 1,942 & -- & 4,654 & -- \\
\hline Other hardwoods & $\ldots$ & 681 & 5,135 & 640 & 6,983 & 2,948 & 8,656 & 1,419 \\
\hline Noncommercial species & - & 660 & 8,384 & -- & 1,463 & 385 & 868 & -- \\
\hline Total & 7,084 & 42,146 & 133,960 & 106,663 & 100,223 & 140,234 & 96,397 & 27,827 \\
\hline All species & 107,673 & 64,418 & 133,960 & 111,564 & 101,799 & 143,442 & 99,553 & 33,860 \\
\hline
\end{tabular}




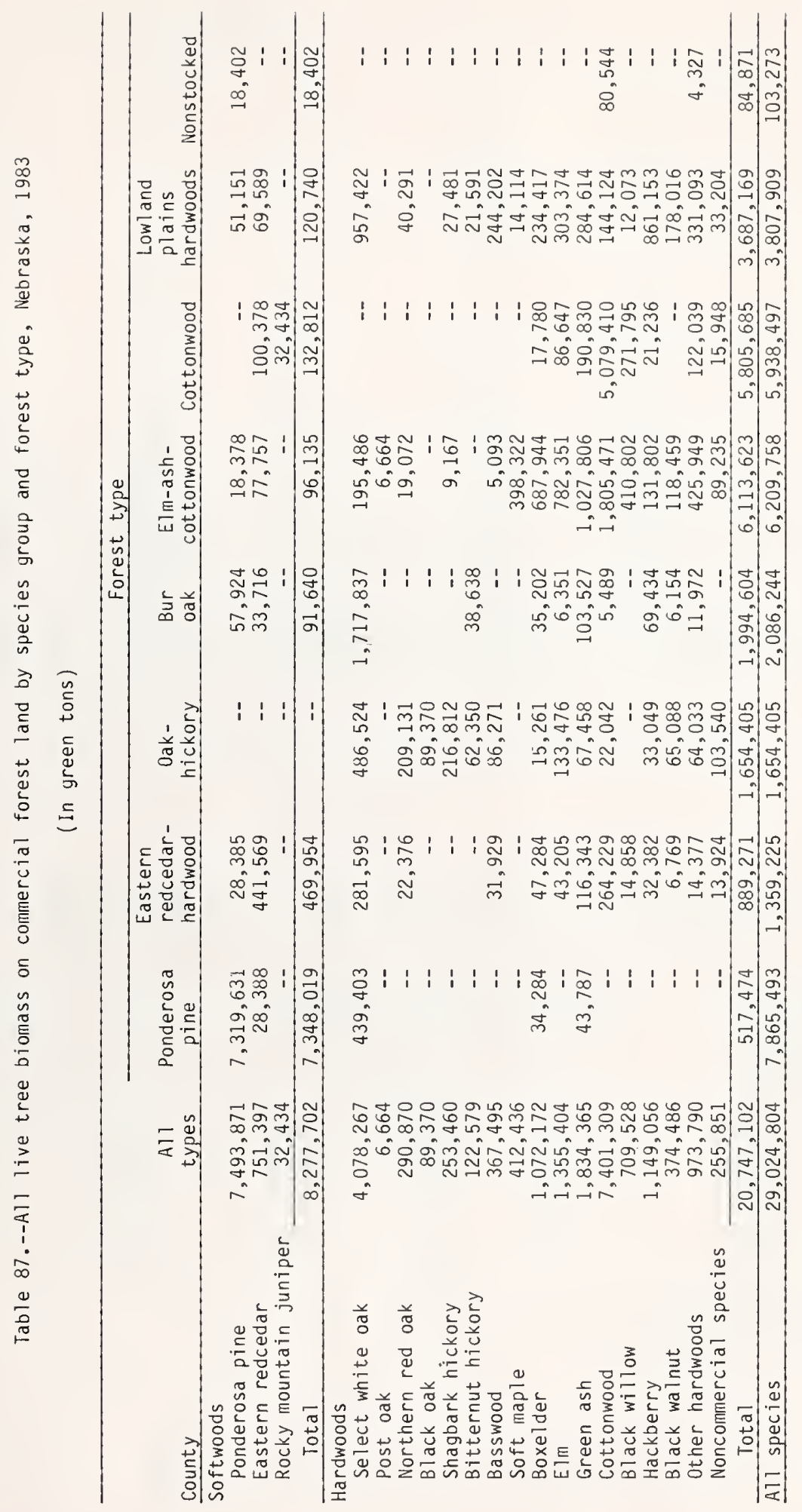


Table 88.--All live tree biomass weight on commercial forest land by species group and tree biomass component, Nebraska, 1983

(In green tons)

\begin{tabular}{|c|c|c|c|c|c|c|}
\hline \multirow[b]{3}{*}{ Species group } & \multirow[b]{3}{*}{$\begin{array}{c}\text { Al1 } \\
\text { components }\end{array}$} & \multicolumn{5}{|c|}{ Biomass component } \\
\hline & & \multirow[b]{2}{*}{$\begin{array}{l}1-\text { to } 5 \text {-inch } \\
\text { trees }\end{array}$} & \multicolumn{2}{|c|}{ Growing stock } & \multicolumn{2}{|c|}{ Cul1 } \\
\hline & & & Boles & $\begin{array}{c}\text { Tops and } \\
1 \mathrm{imbs}\end{array}$ & Boles & $\begin{array}{c}\text { Tops and } \\
\text { Timbs }\end{array}$ \\
\hline \multirow{5}{*}{$\begin{array}{l}\text { Softwoods } \\
\text { Ponderosa pine } \\
\text { Eastern redcedar } \\
\text { Rocky mountain juniper } \\
\text { Total } \\
\end{array}$} & & & & & & \\
\hline & $7,493,871$ & 455,183 & $4,441,953$ & $1,914,849$ & 518,649 & 163,237 \\
\hline & 751,397 & 369,165 & 193,225 & 78,397 & 90,441 & 20,169 \\
\hline & 32,434 & -- & $\ldots$ & $\ldots$ & 23,110 & 9,324 \\
\hline & $8,277,702$ & 824,348 & $4,635,178$ & $1,993,246$ & 632,200 & 192,730 \\
\hline \multicolumn{7}{|l|}{ Hardwoods } \\
\hline Select white oak & $4,078,267$ & 206,659 & $1,607,676$ & 665,797 & $1,153,468$ & 444,667 \\
\hline Post oak & 6,664 & - & 4,769 & 1,895 & -- & -- \\
\hline Northern red oak & 290,870 & -- & 182,065 & 76,896 & 22,513 & 9,396 \\
\hline Black oak & 89,370 & 26,438 & 23,508 & 9,751 & 21,023 & 8,650 \\
\hline Shagbark hickory & 253,460 & 76,252 & 110,133 & 45,700 & 15,111 & 6,264 \\
\hline Bitternut hickory & 122,579 & 18,183 & 62,482 & 26,005 & 11,560 & 4,349 \\
\hline Basswood & 367,495 & 22,644 & 177,543 & 74,005 & 66,358 & 26,945 \\
\hline Soft maple & 412,436 & 38,905 & 168,779 & 70,095 & 95,105 & 39,552 \\
\hline Boxelder & $1,072,172$ & 26,769 & 179,021 & 73,835 & 589,705 & 202,842 \\
\hline Elm & $1,355,404$ & 365,497 & 446,737 & 183,223 & 271,703 & 88,244 \\
\hline Green ash & $1,834,365$ & 165,096 & 839,456 & 347,322 & 348,685 & 133,806 \\
\hline Cottonwood & $7,401,309$ & 72,398 & $4,871,130$ & $2,035,833$ & 313,736 & 108,212 \\
\hline Black willow & 709,528 & 48,485 & 399,120 & 162,153 & 72,447 & 27,323 \\
\hline Hackberry & $1,149,056$ & 132,470 & 591,317 & 241,813 & 130,700 & 52,756 \\
\hline Black walnut & 374,486 & 41,055 & 180,941 & 74,518 & 55,679 & 22,293 \\
\hline Other hardwoods & 973,790 & 135,743 & 242,034 & 100,571 & 362,562 & 132,880 \\
\hline Noncommercial species & 255,851 & -- & $\therefore$ & --- & 204,221 & 51,630 \\
\hline Total & $20,747,102$ & $1,376,594$ & $10,086,711$ & $4,189,412$ & $3,734,576$ & $1,359,809$ \\
\hline All species & $29,024,804$ & $2,200,942$ & $14,721,889$ & $6,182,658$ & $4,366,776$ & $1,552,539$ \\
\hline
\end{tabular}

Table 89.--Sampling errors $1 /$ for estimates smaller than the State totals of volume, net growth, removals, and area of commercial forest land, Nebraska, 1983

\begin{tabular}{|c|c|c|c|c|c|c|c|}
\hline \multirow{2}{*}{$\begin{array}{l}\text { Sampling } \\
\text { error } \\
\text { (percent) }\end{array}$} & \multirow{2}{*}{$\begin{array}{l}\text { Commercial } \\
\text { forest area }\end{array}$} & \multicolumn{3}{|c|}{ Growing Stock } & \multicolumn{3}{|c|}{ Sawtimber } \\
\hline & & Inventory & Growth & Removals & Inventory & Growth & Removals \\
\hline & $\frac{\text { Thousand }}{\text { acres }}$ & - - - MiI & cubic & $=---$ & - - -Mill & board $f$ & 2) - - - \\
\hline $\begin{array}{r}5 \\
10 \\
15 \\
20 \\
25 \\
50 \\
100\end{array}$ & $\begin{array}{r}784.2 \\
196.0 \\
87.1 \\
49.0 \\
31.4 \\
7.8 \\
2.0\end{array}$ & $\begin{array}{r}1,361.6 \\
340.4 \\
151.3 \\
85.1 \\
54.5 \\
13.6 \\
3.4\end{array}$ & $\begin{array}{r}52.8 \\
13.2 \\
5.9 \\
3.3 \\
2.1 \\
0.5 \\
0.1\end{array}$ & $\begin{array}{r}158.2 \\
39.5 \\
17.6 \\
9.9 \\
6.3 \\
1.6 \\
0.4\end{array}$ & $\begin{array}{r}6,009.7 \\
1,502.4 \\
667.7 \\
375.6 \\
240.4 \\
60.1 \\
15.0\end{array}$ & $\begin{array}{r}351.5 \\
87.9 \\
39.1 \\
22.0 \\
14.1 \\
3.5 \\
0.9\end{array}$ & $\begin{array}{r}633.6 \\
158.4 \\
70.4 \\
39.6 \\
25.3 \\
6.3 \\
1.6\end{array}$ \\
\hline
\end{tabular}

$1 /$ At the 68-percent probability level.

2/ International $1 / 4$-inch rule. 
Raile, Gerhard K.

Nebraska forest statistics, 1983. Resour. Bull. NC-91. St. Paul, MN: U.S. Department of Agriculture, Forest Service, North Central Forest Experiment Station; 1985. 70 p.

The second forest resource inventory of Nebraska showed a 25 percent decline in commercial forest area and 23 percent gain in growing-stock volume between 1955 and 1983.

KEY WORDS: Area, volume, growth, mortality, removals. 
